\title{
Thinking beyond numbers
}

Citation for published version (APA):

Backhaus, R. (2017). Thinking beyond numbers: nursing staff and quality of care in nursing homes.

[Doctoral Thesis, Maastricht University]. Datawyse / Universitaire Pers Maastricht.

https://doi.org/10.26481/dis.20170412rb

Document status and date:

Published: 01/01/2017

DOI:

10.26481/dis.20170412rb

Document Version:

Publisher's PDF, also known as Version of record

\section{Please check the document version of this publication:}

- A submitted manuscript is the version of the article upon submission and before peer-review. There can be important differences between the submitted version and the official published version of record.

People interested in the research are advised to contact the author for the final version of the publication, or visit the DOI to the publisher's website.

- The final author version and the galley proof are versions of the publication after peer review.

- The final published version features the final layout of the paper including the volume, issue and page numbers.

Link to publication

\footnotetext{
General rights rights.

- You may freely distribute the URL identifying the publication in the public portal. please follow below link for the End User Agreement:

www.umlib.nl/taverne-license

Take down policy

If you believe that this document breaches copyright please contact us at:

repository@maastrichtuniversity.nl

providing details and we will investigate your claim.
}

Copyright and moral rights for the publications made accessible in the public portal are retained by the authors and/or other copyright owners and it is a condition of accessing publications that users recognise and abide by the legal requirements associated with these

- Users may download and print one copy of any publication from the public portal for the purpose of private study or research.

- You may not further distribute the material or use it for any profit-making activity or commercial gain

If the publication is distributed under the terms of Article $25 \mathrm{fa}$ of the Dutch Copyright Act, indicated by the "Taverne" license above, 


\section{THINKING BEYOND NUMBERS}

Nursing Staff and Quality of Care in Nursing Homes

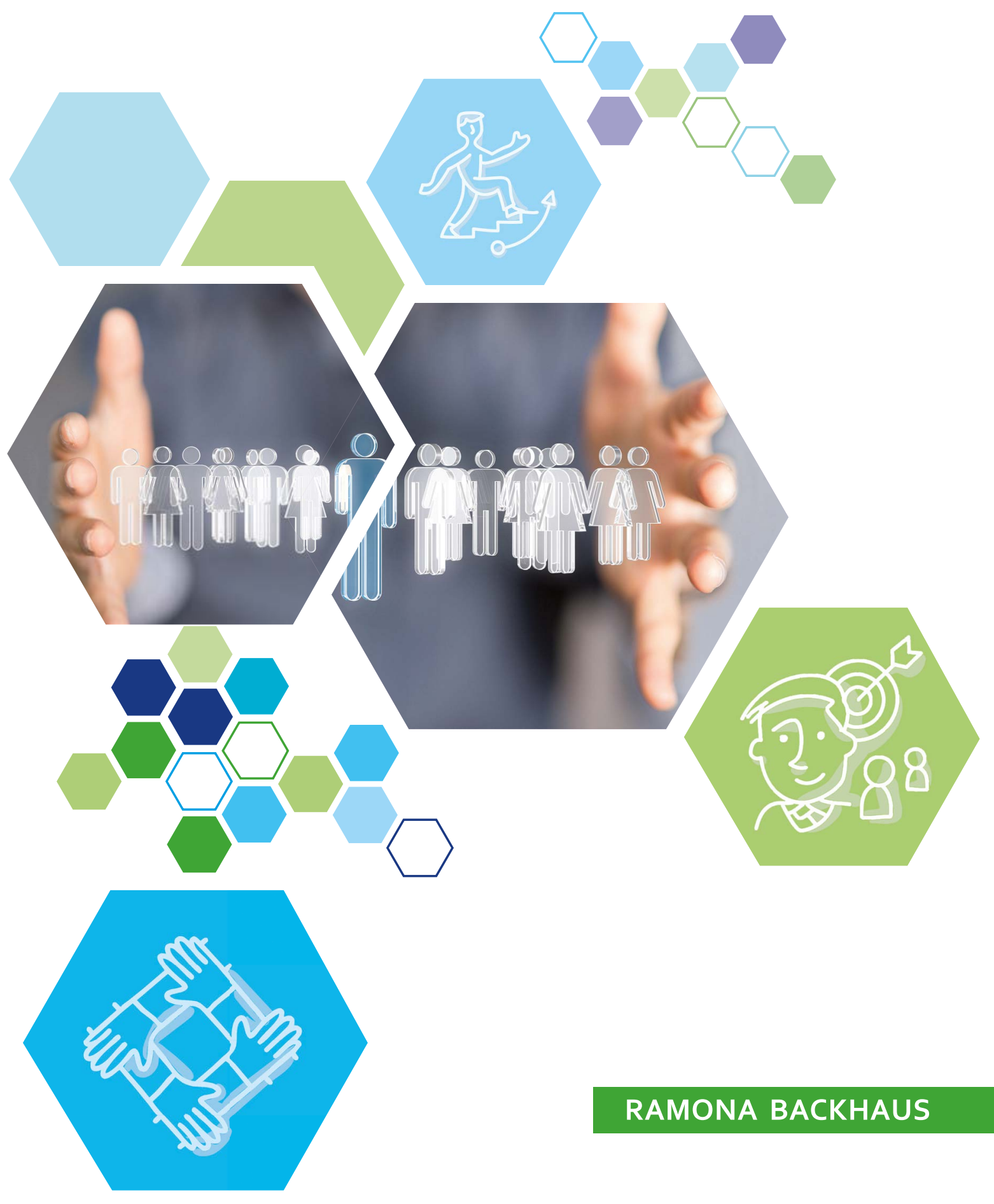


The research presented in this dissertation was part of the research line 'Nurses on the Move: Towards High-Quality Care in Nursing Homes' funded by ZonMw, the Netherlands Organization for Health Research and Development (project number: 520001003).

(C) copyright Ramona Backhaus, Maastricht 2017

Cover: www.deas-printdesign.com

Printing: Datawyse | Universitaire Pers Maastricht

ISBN 9789461596758 


\title{
THINKING BEYOND NUMBERS
}

\section{Nursing Staff and Quality of Care in Nursing Homes}

\author{
DISSERTATION \\ to obtain the degree of Doctor at Maastricht University, \\ on the authority of the Rector Magnificus, Prof. dr. Rianne M. Letschert \\ in accordance with the decision of the Board of Deans, \\ to be defended in public \\ on Wednesday $12^{\text {th }}$ April, 2017, at 16.00 hours \\ by \\ Ramona Backhaus
}




\section{SUPERVISORS}

Prof. dr. JPH Hamers

Prof. dr. E Capezuti (City University of New York, US)

\section{CO-SUPERVISORS}

Dr. E van Rossum

Dr. H Verbeek

\section{ASSESSMENT COMMITTEE}

Prof. dr. JMGA Schols (chair)

Prof. dr. DHJM Dolmans

Dr. EJ Finnema (NHL Hogeschool)

Prof. dr. GJ Odekerken-Schröder

Prof. dr. AM Rafferty (King's College London, UK) 


\section{CONTENTS}

CHAPTER 1 General Introduction

CHAPTER 2 Nurse Staffing Impact on Quality of Care in Nursing Homes: A Systematic Review of Longitudinal Studies

Journal of the American Medical Directors Association, 2014

CHAPTER 3 Relationship Between the Presence of Baccalaureate-Educated RNs and Quality of Care: A Cross-Sectional Study in Dutch Long-Term Care Facilities

BMC Health Services Research, 2017

CHAPTER 4 Quantity of Staff and Quality of Care in Dutch Nursing Homes: A CrossSectional Study The Journal of Nursing Home Research Sciences, 2016

CHAPTER 5 Work Environment Characteristics Associated With Quality of Care in Dutch Nursing Homes: A Cross-Sectional Study International Journal of Nursing Studies, 2017

CHAPTER 6 Future Distinguishing Competencies of Baccalaureate-Educated Registered Nurses in Nursing Homes

Geriatric Nursing, 2015

CHAPTER 7 Blazing a Trail for Baccalaureate-Educated Registered Nurses in Nursing Homes: Experiences of Administrators and Nursing Staff Submitted for publication

Summary

Samenvatting

Zusammenfassung

Valorization

Acknowledgements

About the author

List of publications 

CHAPTER 1

GENERAL INTRODUCTION

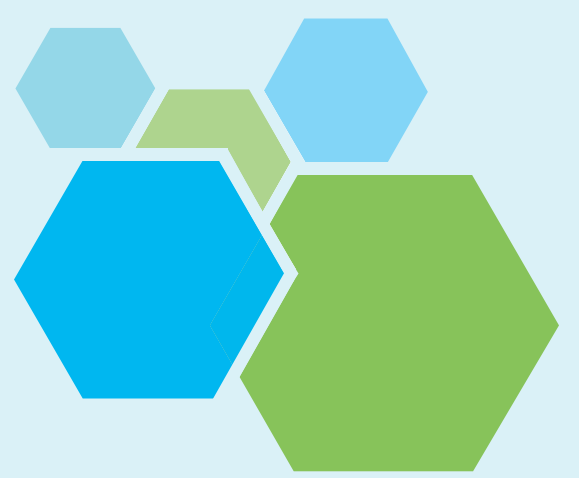



In many countries, poor quality of care $(\mathrm{Q} O \mathrm{C})$ in nursing homes is a concern of older people, care providers and governments. ${ }^{1}$ Complaints and even false perceptions about nursing home QoC are frequently spread in the media. For example, in November 2016, 'pee contracts' were discussed in the Dutch media. This discussion was based on a rumor that nursing home residents in a nursing home had to sign a contract stating that they were allowed to visit the toilet on only three scheduled moments a day. ${ }^{2}$ A 'black list' disseminated in July 2016 reporting the names of 150 Dutch nursing homes that were under increased supervision from the Healthcare Inspectorate received considerable public attention. ${ }^{3}$ This negative publicity is nothing new to the Dutch nursing home sector, as a decade ago, 'pajama days ${ }^{4}$ or nursing homes as 'the waiting room for death' ${ }^{5}$ were discussed in the media.

In moments of media attention, it is often claimed that 'more hands' are needed to improve QoC in nursing homes. ${ }^{6}$ An increase in the number of staff is presumed to positively affect the QoC and quality of life of nursing home residents. ${ }^{7}$ However, so far, research on the relationship between direct nursing care staffing levels has focused primarily on the hospital setting. Evidence from this sector suggests, for example, that an increase in better-educated staff (i.e., baccalaureate-educated registered nurses (BRNs)) leads to better QoC in hospitals. ${ }^{8}$ However, compared to the hospital sector, direct nursing care staff in nursing homes tends to be less educated, ${ }^{9}$ and nursing home organizations in many countries experience difficulties in attracting and retaining registered nurses. ${ }^{10-12}$ Reasons for this are, for example, that working in nursing homes is associated with a low status career and inadequate salaries. ${ }^{13,14}$ Differences in the workforce as well as in the care settings mean that evidence from the hospital sector cannot simply be translated to the nursing home sector. Therefore, more evidence on the relationship between direct nursing care staffing levels and QoC in nursing homes is needed.

The aim of this dissertation is to examine the influence of direct nursing care staff on QoC in nursing homes. In this chapter, the central concepts of this dissertation, i.e., 'nursing homes', 'direct nursing care staff' and 'quality of care' are introduced. At the end of this chapter, the aim and outline of this dissertation are presented.

\section{NURSING HOMES}

In many countries, only the most frail and dependent older adults enter nursing homes. This is related to the preference of older adults to live independently in their own home as long as possible, but also to governmental policies aimed at enabling 'aging in place' and avoiding nursing home admission, especially in European countries. ${ }^{15}$ Although nursing homes exist worldwide, the definition varies within and between countries. To enable comparison across countries, the following international definition for 'nursing 
homes' was presented in 2015 in the Journal of the American Medical Directors Association:

'A nursing home is a facility with a domestic-styled environment that provides 24hour functional support and care for persons who require assistance with activities of daily living (ADLS) and who often have complex health needs and increased vulnerability. Residency within a nursing home may be relatively brief for respite purposes, short term (rehabilitative), or long term, and may also provide palliative/hospice and end-of-life care. In general, most nursing homes also provide some degree of support from health professionals, but [...] a small subset provide socialization activities and basic assistance with ADLs but have no trained health professionals on staff. Although post-acute rehabilitation may be provided in the nursing home (i.e., in the United States and The Netherlands), in many countries this is provided in separate facilities (i.e., geriatric or cottage hospitals) or in a geriatric unit of the acute hospital. ${ }^{16}$

This definition illustrates that, within and between countries, there is much variety regarding the care provided in nursing homes. In addition, the professions that work in nursing homes may vary by country. ${ }^{16}$ In the Netherlands, long-term care for older adults is typically provided in somatic (for residents with physical disabilities) or psychogeriatric (for residents with dementia) wards. ${ }^{17}$ Additionally, Dutch nursing homes provide short-term care for rehabilitation purposes (in rehabilitation wards), as well as crisis intervention and respite care services. ${ }^{18-20}$ In this dissertation, only longterm nursing care wards will be considered. The goal of long-term care wards is to provide a supportive, safe and homelike environment for residents, while assisting them to maintain their functional abilities for as long as possible. ${ }^{16}$ While the goal is the same for all Dutch long-term nursing home wards, the physical environment, however, varies, ranging from traditional large-scale wards, small-scale, homelike wards, to more innovative types like green care farms. ${ }^{21}$ Unique for the Netherlands is that specifically trained nursing home medical specialists provide medical care for nursing home residents. ${ }^{16,17}$ These specialists and all other health professionals (e.g., psychologists, physiotherapists) are employed by the nursing home organization. Most of the roundthe-clock direct care in nursing homes is provided by direct nursing care staff, including registered nurses, certified nurse assistants and nurse aides.

Despite the heterogeneity across countries, nursing homes worldwide have to ensure the delivery of high $\mathrm{QoC}$, while adequately staffing the homes remains a major concern in most countries. Direct nursing care staff vary in their educational level, thus it is a significant challenge to determine the numbers and type of staff as well as staff's competencies that are necessary to meet the complex needs of nursing home residents. ${ }^{9}$ 


\section{DIRECT NURSING CARE STAFF}

Worldwide, direct nursing care staff forms the largest group of employees in nursing homes. Across countries, the educational background of direct nursing care staff differs substantially. For example, in the United States, a minimum of 75 hours of initial training is required to become a certified nurse assistant. ${ }^{22}$ In the Netherlands, in contrast, the educational program to become a nurse assistant is two years. Table 1 provides an overview about direct nursing care staff in Dutch nursing homes. In general, direct nursing care staff can be classified into registered nurses (RNs), licensed nurses or nurse assistants. ${ }^{23}$

In most reported studies, the educational background of RNs is not further clarified, while in most countries two types of RNs can be distinguished: those that hold a diploma or degree at the baccalaureate-level, and those that are vocationally trained. In the Netherlands, baccalaureate degrees can be achieved at universities of applied sciences, while in other countries, these degrees might be offered at various types of academic institutions. A new educational program in the Netherlands offers BRNs the opportunity to specialize in gerontology and geriatrics. ${ }^{24}$ The program length to become a (B)RN in the Netherlands is four years. In Dutch, BRNs are called 'HBOverpleegkundigen', and RNs are called 'MBO-verpleegkundigen'.

Licensed nurses receive vocational training. In the United States, they are called 'licensed practical nurses' or 'licensed vocational nurses'. Other countries refer to 'certified nurse assistants' when talking about licensed nurses. ${ }^{25}$ The program length to become a certified nurse assistant ('verzorgende' in Dutch), is two to three years. Different types of nurse assistants can be distinguished, who are all less educated than the licensed nurses. In the international literature, they are, for example, called 'nurse assistants', 'nurse aides', or 'direct care workers'. In the Netherlands, we can distinguish between nurse assistants ('helpende' in Dutch) and nurse aides ('zorghulp' in Dutch).

Table 1: Direct nursing care staff in Dutch nursing homes.

\begin{tabular}{llcc}
\hline English title & Dutch title & $\begin{array}{l}\text { Dutch qualification } \\
\text { level* }\end{array}$ & $\begin{array}{c}\text { Training length } \\
\text { (in years) }\end{array}$ \\
\hline Baccalaureate-educated registered nurse & HBO-verpleegkundige & 6 & 4 \\
Vocationally-trained registered nurses & MBO-verpleegkundige & 4 & 4 \\
Certified nurse assistant & Verzorgende & 3 & $2-3$ \\
Nurse assistant & Helpende & 2 & 2 \\
Nurse aide & Zorghulp & 1 & $0.5-1$ \\
\hline
\end{tabular}

Note: * According to Dutch qualifications framework (NLQF) 
In addition to the registered nurses, licensed nurses and nurse assistants, other staff members may work in direct care in nursing homes, too. For example, nursing homes may employ trainees or untrained staff. In Dutch nursing homes, often, specifically trained feeding assistants are employed to work in direct resident care. While we will pay specific attention to the employment of BRNs in nursing homes, we will also refer to 'total direct care staff'. In this dissertation, direct care staff may consist of: BRNs, vocationally trained RNs, certified nurse assistants, nurse assistants, nurse aides, feeding assistants, trainees and untrained staff. The general belief is that not only a higher number of total staff hours per resident day (HPRD) is related to better QoC in nursing homes, but also a better staff mix (higher \% registered nurses/total staff). Nevertheless, evidence for these hypotheses is scarce. ${ }^{9,26,27}$

\section{QUALITY OF CARE}

Quality of Care (QoC) is a multidimensional concept and several definitions of QoC exist. ${ }^{6,28}$ According to the Institute of Medicine, QoC can be defined as the degree to which health services for individuals and populations increase the likelihood of desired health outcomes and are consistent with current professional knowledge'. ${ }^{29}$ The World Health Organization defines six areas or dimensions of QoC: effectivity, efficiency, accessibility, acceptability/patient-centeredness, equitability, and safety. ${ }^{30}$ Nowadays, the measurement of QoC in nursing homes is often based on Donabedian's quality paradigm. $^{31}$ In his structure, process, and outcome (SPO) framework, QoC is operationalized in three domains. ${ }^{32}$ Structural attributes of QoC are, for example, related to human resources (e.g., staffing levels), the organizational structure (e.g., payment system) or material resources (e.g., technological equipment). Process denotes to what is actually done (e.g., working according to evidence-based protocols). Outcome refers to health status of patients or residents. ${ }^{6,32}$ Assessment instruments like the resident assessment instrument (RAI) or minimum data set (MDS) cover Donabedian's SPO-domains, and are used in the United States and some European countries to assess the health status of nursing home residents. ${ }^{6}$

In the end, the choice of $\mathrm{QoC}$ indicators may influence the perception of nursing home quality, as nursing homes with good outcomes on some indicators may perform poorly on others. ${ }^{6}$ While traditionally $\mathrm{QOC}$ is assessed based on clinical indicators measuring adverse events (e.g., pressure ulcers, fall incidents), ${ }^{9}$ nowadays there is a tendency to consider staff-, family- or resident-reported QoC indicators as well. ${ }^{6,33}$ Nevertheless, up until now, few studies exist that examine the relationship between staffing and staff-, family- or resident-reported QoC in nursing homes. ${ }^{34}$ 


\section{AIM AND OUTLINE}

Aim

The aim of this dissertation is to examine the influence of direct nursing care staff on QoC in nursing homes. Besides considering staffing levels, particular attention is paid to the competencies, tasks and employment of BRNs, as they are expected to serve as informal leaders with the ability to lead improvements and redesign practice environments in nursing homes. More specifically, this dissertation provides insight into 1) the relationship between direct nursing care staffing and staff-related work environment characteristics and QoC in nursing homes; 2) future desirable distinguishing competencies of BRNs nursing homes; and 3) how organizations employ BRNs in nursing homes and what is the added value they bring to practice.

\section{Outline}

Chapter 2 presents the results of a systematic review on longitudinal studies examining the relationship between nurse staffing and $\mathrm{QoC}$ in nursing homes. Chapter 3 reports on a cross-sectional study on the relationship between the presence of BRNs and QoC conducted among 282 wards and 6,145 residents from 95 Dutch long-term care facilities. The results of a cross-sectional study assessing the relationship between HPRD and QoC in 55 Dutch nursing home wards are provided in Chapter 4. Chapter 5 presents a cross-sectional study examining the relationship between direct care staffing levels (measured as HPRD), work environment characteristics and staff-perceived QoC in 55 Dutch nursing home wards. The aim of the study presented in Chapter 6 was to reach consensus on competencies, which should in the future, distinguish BRNs from other nursing staff (e.g., vocationally trained registered nurses, certified nurse assistants) in nursing homes. Chapter 7 reports on a qualitative study, aimed at obtaining insight into how organizations employ BRNs in nursing homes and what is the perceived added value in care practices that organizations experience from their employment. The final chapter (Chapter 8) summarizes the main findings of our studies, discusses methodological and theoretical considerations, and presents future directions for practice and research. 


\section{RESEARCH LINE 'NURSES ON THE MOVE: TOWARDS HIGH-QUALITY CARE IN NURSING HOMES' AND LIVING LAB IN AGEING \& LONG-TERM CARE}

This PhD project is part of the research line 'Nurses on the Move: Towards High-Quality Care in Nursing Homes' funded by ZonMw, the Netherlands Organization for Health Research and Development (project number: 520001003). The overall objective of this research line is to contribute to the improvement of QoC in nursing homes in general, by improving the functional status of and reducing disability in nursing home residents. The research line consists of three interrelated PhD projects. In project one, a care approach is developed to encourage physical activity and improve the functional status of residents. Project two aims to support nursing staff in implementing innovations. In project three (described in this dissertation), direct nursing care staffing and QoC in nursing homes are studied.

The research line is embedded in the Living Lab in Ageing \& Long-Term Care. This is a formal, multidisciplinary network consisting of Maastricht University, Zuyd University of Applied Sciences and seven large long-term care organizations, all located in the southern part of the Netherlands. The aim of this network is to improve the quality of long-term care. 


\section{REFERENCES}

1. Schwendimann R, Zúñiga F, Ausserhofer D, Schubert M, Engberg S, De Geest S. Swiss Nursing Homes Human Resources Project (SHURP): protocol of an observational study. J Adv Nurs. 2014;70:915-926.

2. Kuiken A. Verpleeghuis beveiligd na bedreigingen. Trouw. November 3, 2016: 5.

3. Redecatie Gezondheid \& Zorg. Staatssecretaris komt met lijst van goede en slechte huizen. Trouw. July 5, 2016: 5 .

4. Van Veen R. ledere dag douchen doet te veel pijn. Trouw. October 7, 2004: 15.

5. Boekholdt M. Zorgen om de zorg in verpleeghuizen: het verpleeghuis voorbij. Tijdschr Gerontol Geriatr. 2005;36:51-54

6. Giorgio LD, Filippini M, Masiero G. Is higher nursing home quality more costly? Eur J Health Econ. 2016;17:1011-1026.

7. Mueller C, Arling G, Kane R, Bershadsky J, Holland D, Joy A. Nursing home staffing standards: their relationship to nurse staffing levels. Gerontologist. 2006;46:74-80.

8. Aiken LH, Sloane DM, Bruyneel L, et al. Nurse staffing and education and hospital mortality in nine European countries: a retrospective observational study. Lancet. 2014;383:1824-1830.

9. Spilsbury K, Hewitt C, Stirk L, Bowman C. The relationship between nurse staffing and quality of care in nursing homes: A systematic review. Int J Nurs Stud. 2011;48:732-750.

10. McGilton KS, Bowers BJ, Heath $\mathrm{H}$, et al. Recommendations from the international consortium on professional nursing practice in long-term care homes. J Am Med Dir Assoc. 2016;17:99-103.

11. Chenoweth L, Jeon YH, Merlyn T, Brodaty $H$. A systematic review of what factors attract and retain nurses in aged and dementia care. J Clin Nurs. 2010;19:156-167.

12. Chu $\mathrm{CH}$, Wodchis WP, McGilton KS. Turnover of regulated nurses in long-term care facilities. I Nurs Manag. 2014;22:553-562.

13. Eldh AC, Zijpp T, McMullan C, McCormack B, Seers K, Rycroft-Malone J. 'I have the world's best job'-staff experience of the advantages of caring for older people. Scand J Caring Sci. 2016;30:365-373.

14. Phelan A, McCormack B. Exploring nursing expertise in residential care for older people: a mixed method study. J Adv Nurs. 2016;72:2524-2535.

15. Thoma-Lürken T, Bleijlevens MHC, Lexis MAS, Hamers JPH, De Witte CP. An overview of potential laborsaving and quality-improving innovations in long-term care for older people. J Am Med Dir Assoc. 2015;16:482-489.

16. Sanford AM, Orrell M, Tolson D, et al. An international definition for "nursing home". J Am Med Dir Assoc. 2015;16:181-184.

17. Huls M, De Rooij SE, Diepstraten A, Koopmans R, Helmich E. Learning to care for older patients: hospitals and nursing homes as learning environments. Med Educ. 2015;49:332-339.

18. Schols J, Crebolder H, van Weel C. Nursing home and nursing home physician: the Dutch experience. J Am Med Dir Assoc. 2004;5:207-212.

19. Hoek JF, Penninx BW, Ligthart GJ, Ribbe MW. Health care for older persons, a country profile: The Netherlands. J Am Geriatr Soc. 2000;48:214-217.

20. Verbeek H. Redesigning dementia care. Maastricht: Maastricht University; 2011.

21. De Boer B, Hamers JPH, Beerens HC, Zwakhalen SM, Tan FES, Verbeek H. Living at the farm, innovative nursing home care for people with dementia-study protocol of an observational longitudinal study. BMC Geriatr. 2015;15:144.

22. Han K, Trinkoff AM, Storr CL, Lerner N, Johantgen M, Gartrell K. Associations between state regulations, training length, perceived quality and job satisfaction among certified nursing assistants: cross-sectional secondary data analysis. Int J Nurs Stud. 2014;51:1135-1141.

23. Kim H, Harrington C, Greene W. Registered nurse staffing mix and quality of care in nursing homes: a longitudinal analysis. Gerontologist. 2009;49:81-90.

24. Huizenga P, Finnema E, Roodbol P. Learnt and perceived professional roles of a new type of nurse specialized in gerontology and geriatrics, a qualitative study. J Adv Nurs. 2016;72:1552-1566. 
25. Verkaik R, Francke AL, Van Meijel B, Spreeuwenberg PM, Ribbe MW, Bensing JM. The introduction of a nursing guideline on depression at psychogeriatric nursing home wards: effects on certified nurse assistants. Int J Nurs Stud. 2011;48:710-719.

26. Corazzini KN, Anderson RA, Mueller C, Thorpe JM, McConnell ES. Licensed practical nurse scope of practice and quality of nursing home care. Nurs Res. 2013;62:315-324.

27. Castle N. Nursing home caregiver staffing levels and quality of care. J App/ Gerontol. 2008;27:375 - 405.

28. Tolson D, Rolland Y, Andrieu S, et al. International Association of Gerontology and Geriatrics: A Global Agenda for Clinical Research and Quality of Care in Nursing Homes. J Am Med Dir Assoc. 2011;12:184189.

29. Lohr KN. Medicare: a strategy for quality assurance. Washington, DC: National Academies Press; 1990.

30. World Health Organization. Quality of care: a process for making strategic choices in health systems. Geneva: World Health Organization; 2006.

31. Donabedian A. The definition of quality and approaches to its assessment. Ann Arbor, MI: Health Administration Press; 1980.

32. Donabedian A. The quality of care: how can it be assessed? Jama. 1988;260:1743-1748.

33. Ma C, Olds DM, Dunton NE. Nurse work environment and quality of care by unit types: a cross-sectional study. Int J Nurs Stud. 2015;52:1565-1572.

34. Zúñiga F. The significance of staffing and work environment for quality of care and the recruitment and retention of care workers. Perspectives from the Swiss Nursing Homes Human Resources Project (SHURP). Basel: University of Basel; 2015. 


\title{
CHAPTER 2
}

\author{
NURSE STAFFING IMPACT ON \\ QUALITY OF CARE IN \\ NURSING HOMES: \\ A SYSTEMATIC REVIEW OF \\ LONGITUDINAL STUDIES
}

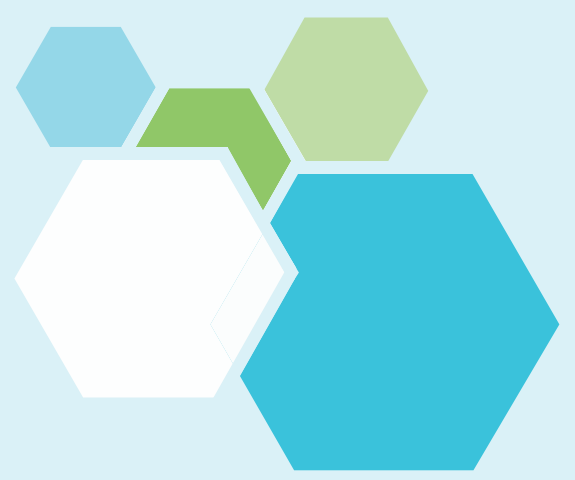

This chapter was published as:

Backhaus R, Verbeek H, Van Rossum E, Capezuti E, Hamers JPH. Nurse Staffing Impact on Quality of Care in Nursing Homes: A Systematic Review of Longitudinal Studies. Journal of the American Medical Directors Association 2014;15:383-393. 


\section{ABSTRACT}

Background: The relationship between nurse staffing and quality of care $(\mathrm{Q} o \mathrm{C})$ in nursing homes continues to receive major attention. The evidence supporting this relationship, however, is weak because most studies employ a cross-sectional design. This review summarizes the findings from recent longitudinal studies.

Methods: In April 2013, the databases PubMed, CINAHL, EMBASE, and PsycINFO were systematically searched. Studies were eligible if they (1) examined the relationship between nurse staffing and QoC outcomes, (2) included only nursing home data, (3) were original research articles describing quantitative, longitudinal studies, and (4) were written in English, Dutch, or German. The methodological quality of 20 studies was assessed using the Newcastle-Ottawa scale, excluding 2 low-quality articles for the analysis.

Results: No consistent relationship was found between nurse staffing and QoC. Higher staffing levels were associated with better as well as lower QoC indicators. For example, for restraint use both positive (ie, less restraint use) and negative outcomes (ie, more restraint use) were found. With regard to pressure ulcers, we found that more staff led to fewer pressure ulcers and, therefore, better results, no matter who (registered nurse, licensed practical nurse/licensed vocational nurse, or nurse assistant) delivered care.

Conclusions: No consistent evidence was found for a positive relationship between staffing and QoC. Although some positive indications were suggested, major methodological and theoretical weaknesses (eg, timing of data collection, assumed linear relationship between staffing and QoC) limit interpretation of results. Our findings demonstrate the necessity for well-designed longitudinal studies to gain a better insight into the relationship between nurse staffing and $\mathrm{QoC}$ in nursing homes. 


\section{INTRODUCTION}

During the last 3 decades, there has been growing concern about nursing home quality in most industrialized countries, ${ }^{1-4}$ with an apparent variability of quality among countries. $^{5}$ Poor quality of nursing home care has often been associated with insufficient staffing levels, ${ }^{6}$ as staffing is presumed to affect the quality of care (QoC) and life of nursing home residents. ${ }^{7}$ In the United States (US), since 1987, federal government regulations have mandated minimum staffing levels. In addition, some US states have introduced additional nurse staffing requirements for nursing homes. ${ }^{7}$ Conspicuously, experts recommended higher staffing standards than those mandated for US nursing homes. ${ }^{8}$ Inconsistent US findings on staffing and quality in nursing homes suggest that further research is needed. ${ }^{9}$

In recent years, the relationship between staffing and QoC in nursing homes has received considerable attention. Reviews of studies reveal only weak evidence about the association between nurse staffing and QoC in nursing homes. ${ }^{6,10,11}$ For example, Bostick et al $^{10}$ describe the functional ability of residents, the prevalence of pressure ulcers, and residents' weight loss as the most nurse sensitive quality indicators with regard to staffing, but consensus on which quality indicators are most nursing sensitive is absent. ${ }^{2}$ Spilsbury et $\mathrm{al}^{11}$ concluded that the existing research evidence demonstrates inconsistent and contradictory findings about the relationship between nurse staffing and the QoC in nursing homes. They included 50 studies, predominantly from the US and with a cross-sectional design, demonstrating provisional evidence that total nurse, registered nurse (RN) and nurse assistant (NA) staffing influenced the QoC for nursing home residents. The authors ${ }^{11}$ underscore the cross-sectional design as a major criticism of prior studies. Conclusions from cross-sectional studies are possibly biased because of unobserved factors that affect nursing home quality, correlating with the explanatory variables used in these studies. ${ }^{12}$ As a result, these designs could account for the weak associations found in prior studies. ${ }^{6,10-12}$

More evidence is needed, especially from longitudinal studies. For this reason, the aim of this study is to review recent longitudinal studies focusing on nurse staffing and QoC outcomes in nursing homes. This will provide more reliable evidence about staffing and QoC in nursing homes, as we expect less inconsistent and contradictory results compared with those obtained in prior reviews. ${ }^{6,10,11}$ The results of this study will contribute to the discussion about implementing minimum staffing standards and will help to determine optimal nurse staffing levels in nursing homes. 


\section{DEFINITIONS}

In our study, 4 different categories of nursing staff will be distinguished: (1) total nurse staff including RNs, licensed practical nurses (LPN)s or licensed vocational nurses (LVN)s, and nurse assistants (NA)s; (2) RN staff; (3) LPN or LVN staff; and (4) NA staff.

Two staffing characteristics, namely 'nurse staffing levels' and 'professional staff mix' will be central in our analysis, as they are considered to influence QoC in nursing homes. ${ }^{13}$ Studies assessing 'nurse staffing levels' focus on numbers of nurses. 'Nurse staffing levels' can be defined as the ratio of (1) nurse staff to residents or (2) nurse hours per resident. ${ }^{10}$ In studies examining 'nurse staffing levels,' each category of nursing staff (total staff, RN staff, LPN/LVN staff, NA staff) is considered separately. However, 'professional staff mix' is measured as a ratio of different staff categories, for example the ratio of RN to total nurse staff or the ratio of RN to LPN and NA staff.

With regard to QoC outcomes, we will distinguish between clinical (eg, pressure ulcers, infections), process-related (eg, restraining, hospitalization), and administrative outcomes (ie, deficiency citations). Nursing home deficiency citations have been widely used as quality indicators in US nursing home studies. ${ }^{14}$ In the US, nursing home deficiency citations are given to those nursing homes that failed to meet at least 1 federal or state QoC requirement. ${ }^{15}$

\section{METHODS}

\section{Search Strategy}

In April 2013, a literature search was performed. The search strategy included terms related to staffing, quality of care, and the place of residence. Search terms with respect to staffing (Staffing OR "Staff mix" OR "RN mix" OR "RN ratio" OR "Skill mix" OR "Staff utilization") were combined using the Boolean operator 'AND' with search terms relating to QoC ("Quality of care" OR "Patient outcomes" OR "Resident outcomes") and terms referring to the place of residence (nursing homes OR residential facilit* OR Long term care facilit* OR "Assisted living" OR "Residential care" OR "Housing for the elderly" OR care homes OR "Long term care setting" OR institutional* OR "Homes for the aged" OR Special care unit*). Articles published between January 2007 and April 2013 were retrieved by searching PubMed, CINAHL, EMBASE and PsycINFO. Longitudinal studies prior to 2008 were extracted from the review conducted by Spilsbury et al, ${ }^{11}$ as they have selected and reviewed 50 out of 13,411 potential studies published from 1980 to 2007. To obtain all relevant articles published in 2007, we decided to include studies published in 2007 in our own search as well. Furthermore, all the bibliographies of included articles were searched for additional references. 


\section{Eligibility Criteria}

Studies were eligible if they (1) examined the relationship between nurse staffing and QoC outcomes, (2) included only nursing home data, (3) were original research articles describing quantitative, longitudinal studies, and (4) were written in English, Dutch, or German.

\section{Study Screening and Data Extraction}

The retrieved articles were managed in an Endnote library (version X6). Two researchers (RB, HV) independently screened titles and abstracts for relevance. After reaching consensus on the results of the independent screening of abstracts, full-text articles were obtained for potentially relevant studies. In addition, the principal author (RB) searched the review by Spilsbury et al $^{11}$ for longitudinal studies. Two members of the research team independently screened the full-text articles and scored them as 'include,' 'possibly include,' or 'exclude.' To reach consensus about the final list of included studies, disagreement between the researchers was discussed and resolved.

The principal researcher (RB) extracted data from all relevant articles using a standardized form specifically developed for the current study. For all included articles, data on the following items were collected: publication type, aims of the study, study method, independent staffing variables, covariates, findings of the study, and potential limitations and recommendations. The extracted data were discussed within the research team.

\section{Methodological Quality}

The methodological quality of included studies was assessed using the NewcastleOttawa scale for assessing the quality of nonrandomized studies ${ }^{16}$ (Table 1). The scale consists of 9 items covering 3 domains: selection (representativeness of the cohort), comparability (controlling for confounders), and outcomes (assessment and follow-up). Two researchers (RB, HV) independently rated the quality of each included study on a scale from 0 stars to 9 stars. Studies were classified into groups of low (less than 6 stars), moderate (6-7 stars), or high (8-9 stars) quality studies. ${ }^{17,18}$ Disagreement between the researchers was discussed to reach consensus. Table 2 shows the final assessments of all included studies. 
Table 1: Tailored Newcastle Ottawa Scale for Assessing the Quality of Nonrandomized Studies

\begin{tabular}{|c|c|}
\hline Domain & Item \\
\hline ection & $\begin{array}{l}\text { (1) Representativeness of the exposed cohort (1 star) } \\
\text { - truly representative of the average person living in an institutional long-term care } \\
\text { setting } \\
\text { - somewhat representative of the average person living in an institutional long-term } \\
\text { care setting } \\
\text { - selected group of persons living in an institutional long-term care setting } \\
\text { - no description of the derivation of the cohort } \\
\text { (2) Selection of the non-exposed cohort (1 star) } \\
\text { - drawn form the same community as the exposed cohort } \\
\text { - drawn from a different source } \\
\text { - no description of the derivation of the non-exposed cohort } \\
\text { (3) Ascertainment of exposure (staffing) (1 star) } \\
\text { - secure record (eg, medical record) } \\
\text { - structured interview } \\
\text { - written self-report } \\
\text { - no description or ascertained in some other way } \\
\text { (4) Demonstration that outcome of interest was not present at baseline (1 star) } \\
\text { - yes } \\
\text { - no }\end{array}$ \\
\hline Comparability & $\begin{array}{l}\text { (5) Comparability of cohorts on the basis of the design or analysis ( } 2 \text { stars) } \\
\text { - study controls for resident characteristics } \$ \text { - } \\
\text { - study controls for facility characteristics }\end{array}$ \\
\hline Outcomes & 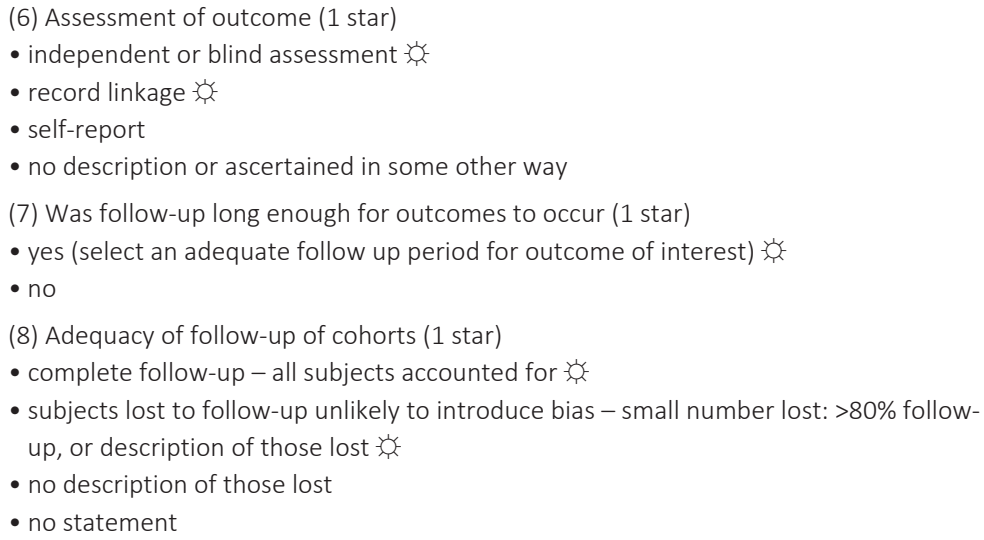 \\
\hline
\end{tabular}

\section{Data Synthesis and Analysis}

Because of the heterogeneity in studies regarding their assessment of nurse staffing characteristics and QoC data, we did not conduct a meta-analysis. Instead, the findings of included studies were summarized in a systematic way. Low-quality studies $(n=2)$ were excluded from analysis. While summarizing the findings, we distinguished studies 
examining 'nurse staffing levels' and 'professional staff mix.' For both categories, the results of included studies were grouped per QoC outcome (eg, pressure ulcers, infections, restraint use). Per QoC outcome, the results were categorized into 4 different groups on the basis of whether they examined (1) total staff, (2) RN staff, (3) LPN/LVN staff, or (4) NA staff. In presenting our findings, we distinguished between studies that found a positive and statistically significant relationship between staffing and QoC outcomes, a negative and statistically significant relationship or no statistically significant association.

\section{RESULTS}

Figure 1 presents the flowchart of the inclusion process. In total, 20 articles were included (Table 2).

\section{General Characteristics of Included Studies}

Table 2 presents an overview of the general characteristics of all included studies. All studies were US-based, ${ }^{13,19-36}$ except 1 study that was conducted in Italy. ${ }^{37}$ Sixteen studies were published from 2002 onwards, 3 studies in 1998 or 1999 25,29,30 and 1 in 1987. ${ }^{31}$

In all included studies, covariates were considered to control for other variables that could potentially affect QoC outcomes. Broadly speaking, these can be grouped into resident (eg, age, sex, disease status), facility (eg, ownership, facility size), and market/economic characteristics (eg, occupancy rates, Medicaid reimbursement rates).

\section{Methodological Differences and Quality of Included Studies}

The included studies differed in the level of analysis used. Six studies $23,30-32,36,37$ conducted analyses on resident-level and 11 studies $^{13,20-22,24,26-28,33-35}$ on facility level. Three studies ${ }^{19,25,29}$ performed multilevel analyses.

Moreover, the included studies differ in the way the staffing-quality relationship was examined. Ten studies ${ }^{13,19,21-24,26-28,31}$ focused specifically on the staffing-quality relationship. The other 10 studies $^{20,25,29,30,32-37}$ used staffing as a control variable. In most included studies, a linear relationship between staffing and QoC outcomes was assumed; the more nursing staff available, the better the quality.

The methodological quality scores of studies ranged from 3 to 9. Two studies ${ }^{19,31}$ were of low quality, $14^{20,22-30,32,34,35,37}$ of moderate quality and $4^{13,21,33,36}$ of high quality. The 2 low-quality articles were excluded from analyses. ${ }^{19,31}$ 


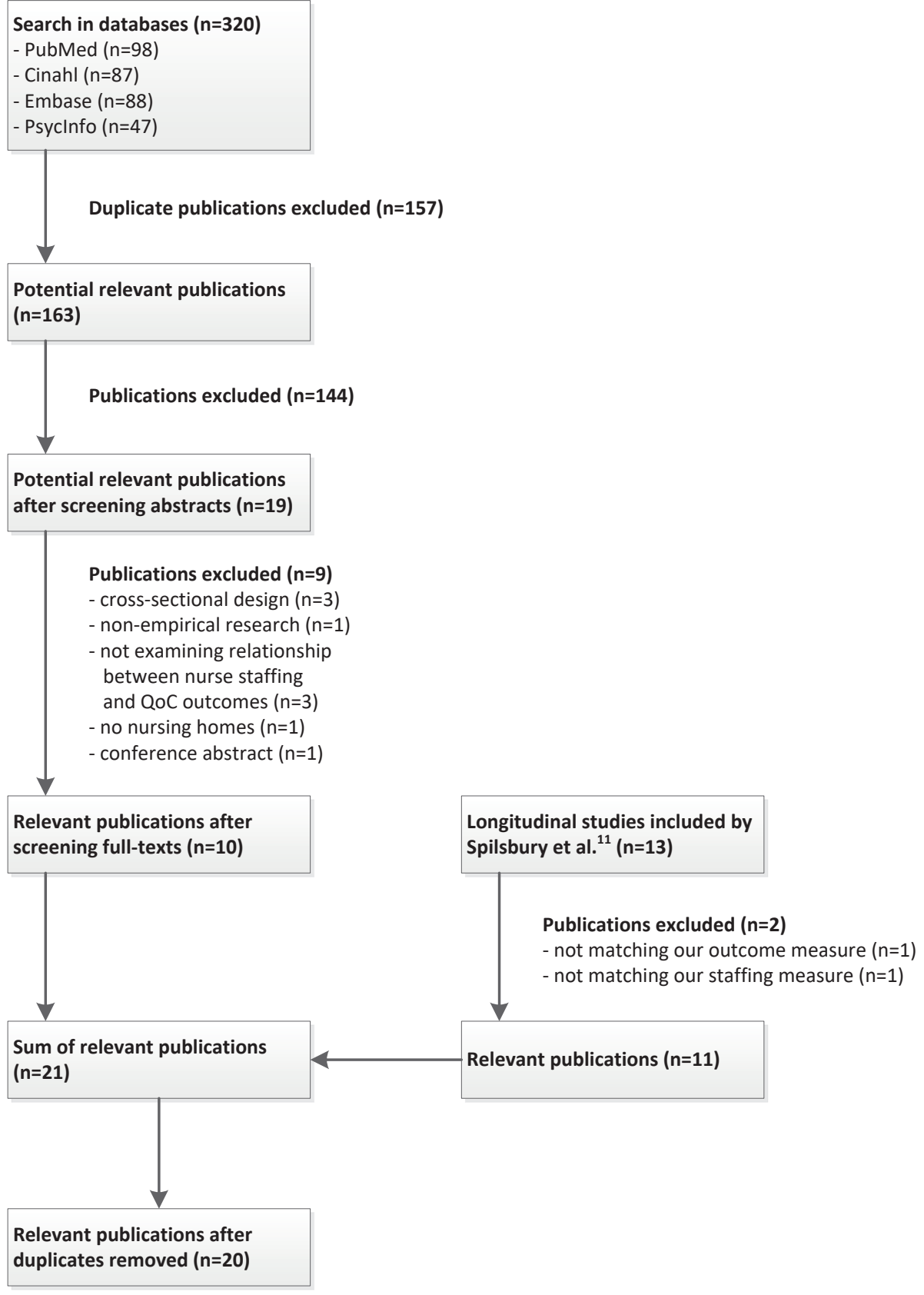

Figure 1: Flow diagram of the search process 












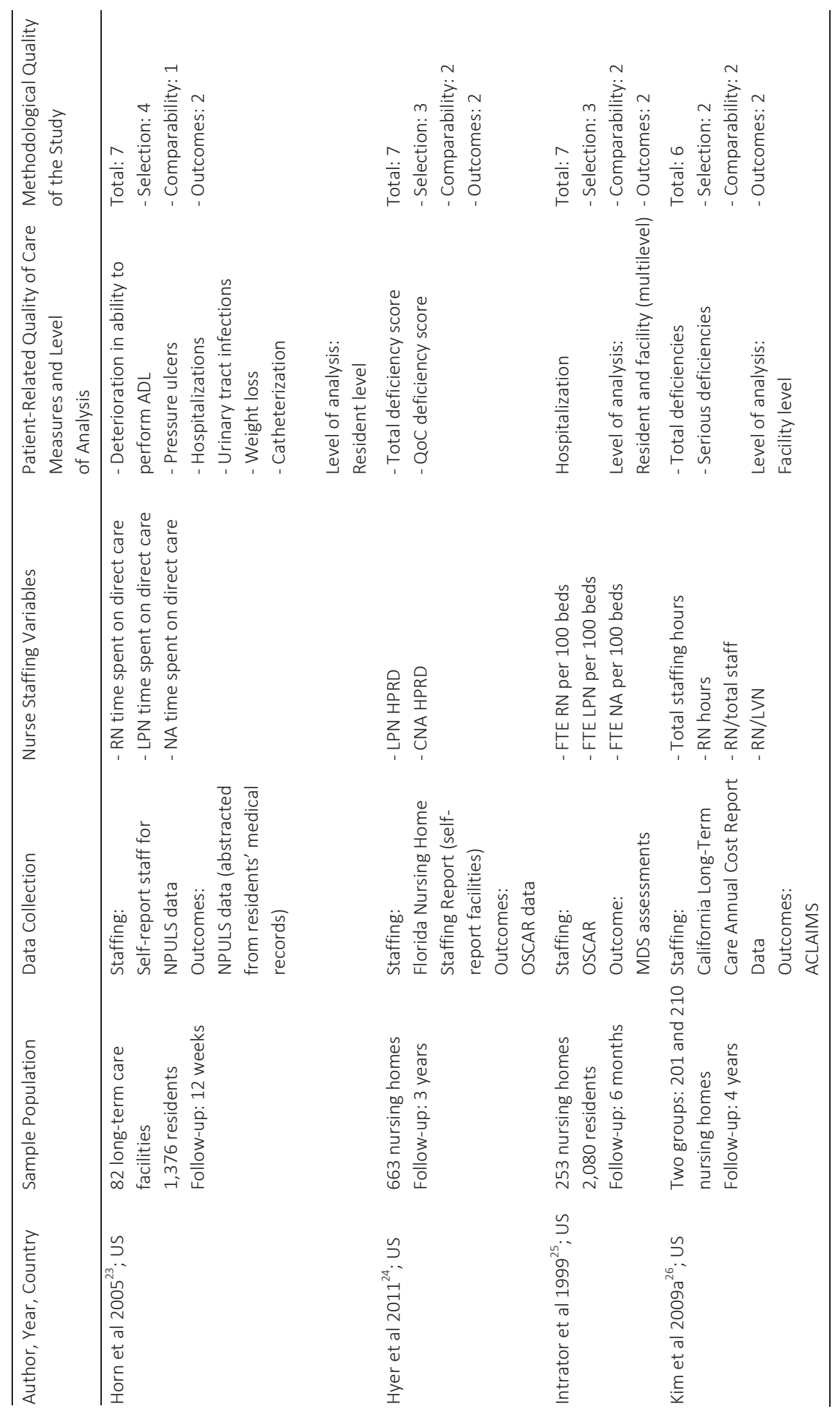




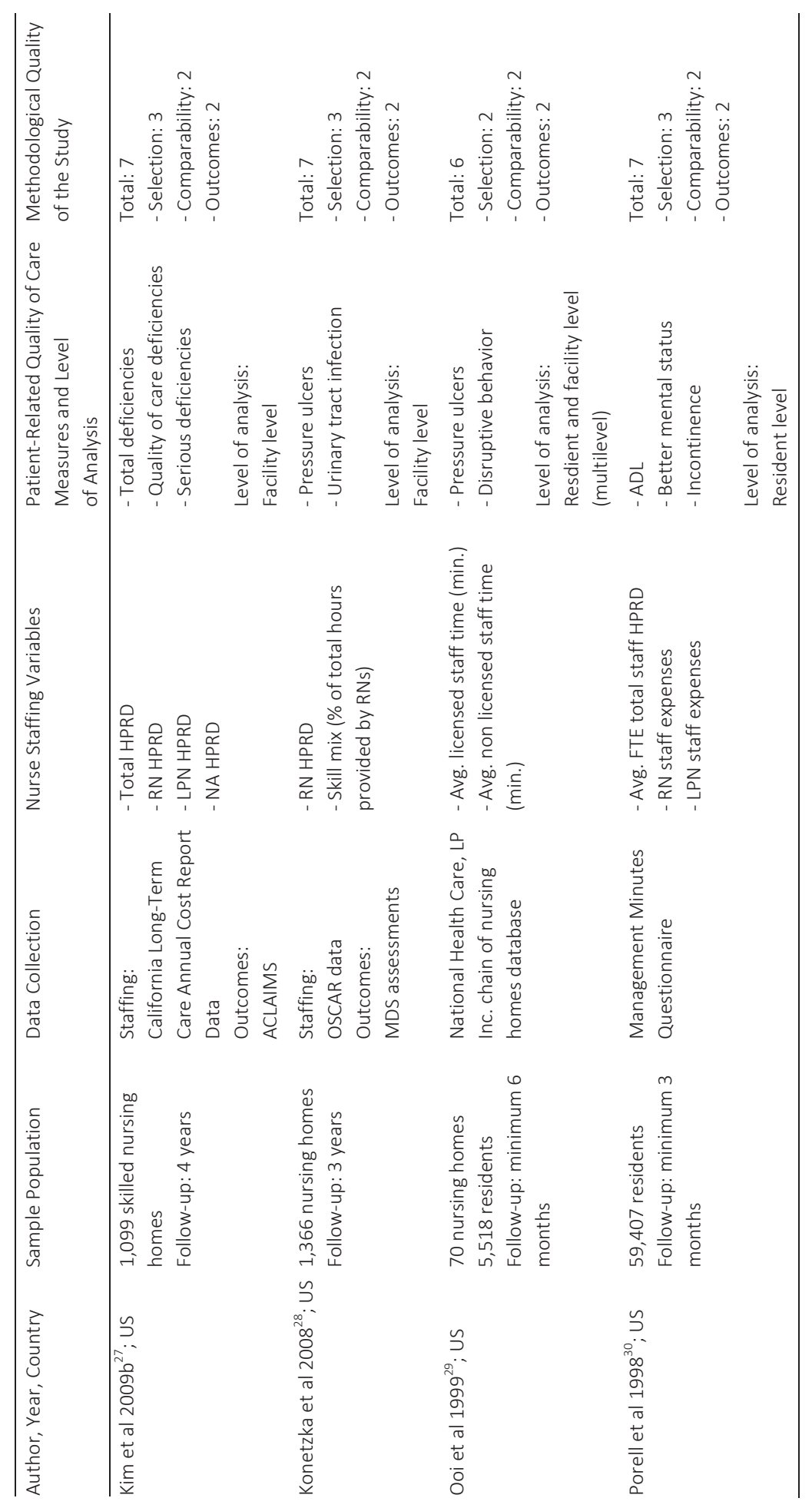


STAFFING AND QUALITY IN NURSING HOMES: A SYSTEMATIC REVIEW






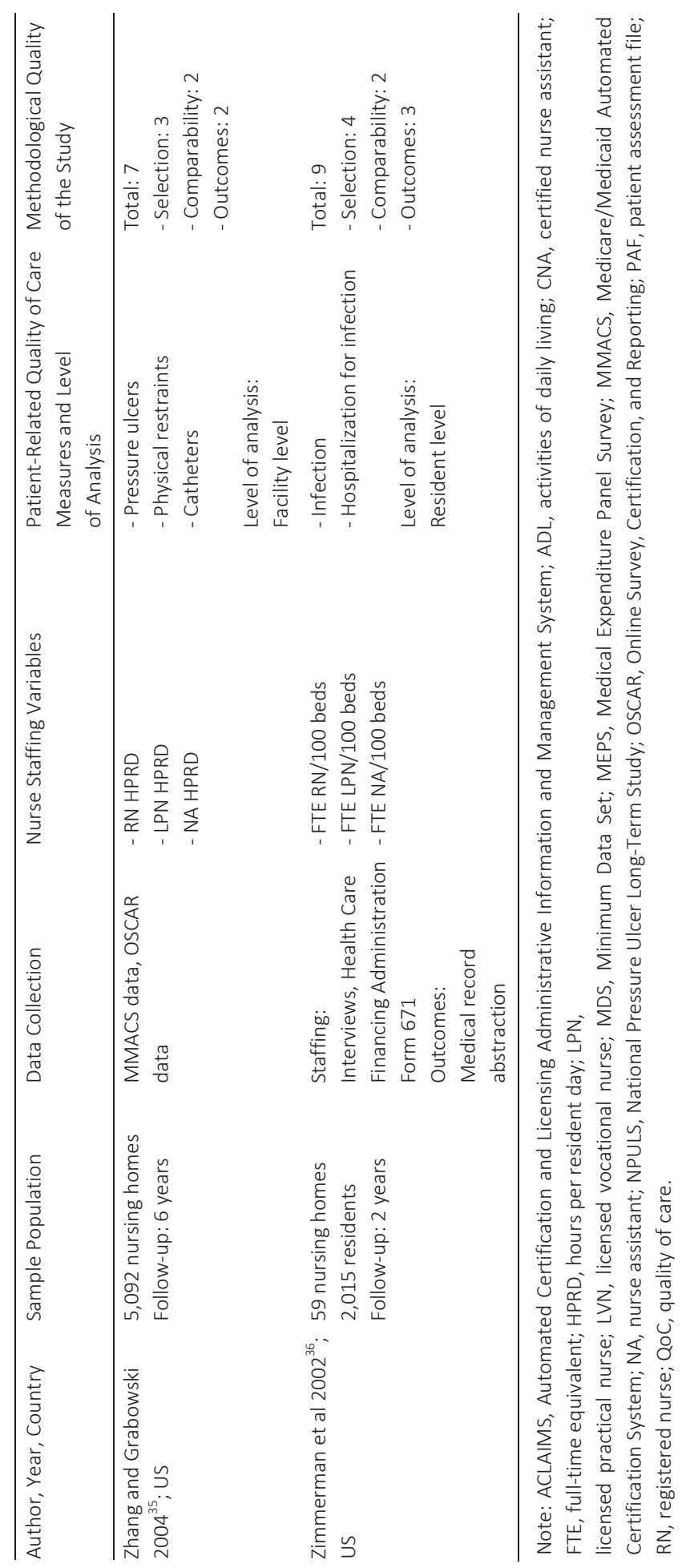




\section{Relationship Between Staffing Levels and QoC Outcomes}

Sixteen studies ${ }^{13,20-25,27,28,30,32-37}$ explored the relationship between nurse staffing levels and QoC outcomes. We distinguish between clinical, process-related, and administrative outcomes for reporting the results. Table 3 summarizes our findings.

\section{Clinical Outcomes}

Nine studies ${ }^{13,22,23,28,30,32,34-36}$ examined the relationship between staffing levels and one or more clinical outcomes. In 1 study, ${ }^{34}$ more total staffing was associated with better clinical outcomes. Three studies ${ }^{13,23,28}$ concluded that more RN staff positively contributed to clinical outcomes, whereas 2 studies $^{30,35}$ found a negative effect of more RN staff. With regard to LPN/LVN staffing, 2 studies ${ }^{13,23}$ associated more staff with better outcomes and $2^{35,36}$ with worse clinical resident outcomes. In 6 studies, ${ }^{13,23,29,32,35,36}$ more NA staffing was associated with fewer clinical problems.

\section{Pressure Ulcers}

Five studies ${ }^{13,22,23,28,35}$ investigated whether there was a relationship between nurse staffing level and the prevalence of pressure ulcers. In general, more RN staff, ${ }^{13,23,28}$ LPN/LVN staff ${ }^{13,23}$ and NA staff ${ }^{13,23,35}$ were associated with a decrease in pressure ulcer prevalence. Contrary, another study ${ }^{35}$ reported that more RN and more LPN staff were associated with an increase in pressure ulcer prevalence. In 1 study, ${ }^{22}$ a significant relationship between more total staff and the pressure ulcer prevalence was absent.

\section{Infections}

The relationship between staffing levels and infections was assessed in three studies. $^{23,28,36}$ Accordingly, more RN staff was linked to fewer urinary tract infections, ${ }^{23,28}$ whereas a significant relationship for LPN/LVN or NA staff was absent. ${ }^{23}$ One study, ${ }^{36}$ which did not focus on a specific type of infections, showed that more LPN/LVN staff was related to a higher occurrence of infections, whereas more NA staff was associated with fewer infections.

\section{Activities of Daily Living Decline}

More RN staff was associated with a decrease $\mathrm{in}^{23}$ or no significant effect ${ }^{30}$ on the likelihood of activities of daily living decline. For total staff, ${ }^{30}$ LPN/LVN, ${ }^{23,30}$ and NA staff, $^{23}$ no significant relationships were observed.

\section{Other Clinical Outcomes}

In a study ${ }^{32}$ investigating the relationship between more NA staff and the occurrence of fractures, more NA staff was associated with fewer fractures. In another study, ${ }^{30}$ more 
RN staff was associated with a higher likelihood of urinary incontinence, whereas a significant relationship with regard to LPN/LVN staff was not found. Another study ${ }^{13}$ showed that higher RN, LPN/LVN, and NA staffing levels were linked to less reported complaints of pain. In another study, ${ }^{23}$ more RN staff was associated with fewer residents losing weight, whereas LPN/LVN and NA staff were not significantly related. One study ${ }^{29}$ found an association between more NA staff and less disruptive behavior of residents. Another study ${ }^{34}$ reported that increasing total staff was associated with an improvement of resident outcomes, measured by weighing the incidents of pressure ulcers, physical restraints, and catheter use.

\section{Process-Related Outcomes}

Six studies ${ }^{13,23,25,35-37}$ examined the relationship between staffing levels and processrelated resident outcomes. More RN staffing was associated with better ${ }^{13,23,37}$ and poorer $^{35}$ process-related outcomes. More LPN/LVN staff led to better ${ }^{13,37}$ or poorer ${ }^{35}$ resident outcomes. Three studies ${ }^{13,35,37}$ reported that more NA staff seemed to be associated with better process-related outcomes.

\section{Restraints}

More RN staff was associated with both more ${ }^{35}$ and less ${ }^{13}$ restraint use. More LPN/LVN staff was associated with less physical restraint use in 1 study, ${ }^{13}$ whereas the other study ${ }^{35}$ did not find a significant relationship. In both studies, ${ }^{13,35}$ it was assumed that in homes with more NA staff, physical restraints were used less often.

\section{Catheterization}

In 3 studies, ${ }^{13,23,35}$ less catheterization was considered as a proxy for a better urinary incontinence status of residents. More RN staff was associated with less ${ }^{13,23}$ as well as with more ${ }^{35}$ catheterizations. One study ${ }^{35}$ reported that catheterization was more likely to occur with more LPN/LVN staff; 2 studies $^{13,23}$ did not find a significant relationship. All 3 studies $^{13,23,35}$ did not find a significant relationship for NA staff.

\section{Hospitalization}

In 4 studies, ${ }^{23,25,36,37}$ hospitalization was considered a proxy for poor overall health. Two studies $^{23,37}$ found that more RN staff was associated with a lower number of hospitalizations, whereas a significant relationship could not be found in another study. ${ }^{25}$ More LPN/LVN staff was associated with fewer hospitalizations in 1 study $^{37}$; in 3 studies, ${ }^{23,25,36}$ no significant relationship was found. More NA staff was associated with a decrease in hospitalization in 1 study, ${ }^{37}$ whereas 3 studies ${ }^{23,25,36}$ did not find a significant relationship. 


\section{Administrative Outcomes}

Five studies ${ }^{20,21,24,27,33}$ focused on the relationship between staffing levels and deficiency citations as QoC outcomes. One study ${ }^{27}$ found that more total staff resulted in fewer deficiency citations. Four studies ${ }^{20,21,27,33}$ reported that more RN staff was associated with fewer deficiency citations, whereas 1 study found a contrary result. ${ }^{20}$ More LPN/LVN staff was associated with more deficiency citations, ${ }^{21,27}$ whereas 1 study ${ }^{33}$ reported the opposite result. Depending on the study analyzed, more NA staff was associated with more ${ }^{21,33}$ or fewer ${ }^{24,27}$ deficiency citations.

In 1 study, ${ }^{27}$ more total staff was associated with a decrease in the amount of total deficiency citations, citations for serious deficiencies and for QoC. Inconsistent results were found for each of the other 3 staffing categories. In another study, ${ }^{21}$ focusing on deficiency citations for safety, more RN staff was associated with a decrease in the number of deficiency citations (adjusted odds ratios: 0.95-0.97), whereas the findings for second-level nurse and NA staff were inconsistent. One study ${ }^{20}$ assessed the relationship between $\mathrm{RN}$, second-level nurse, NA staff and 5 different classifications of deficiency citations for abuse. With regard to RN staff, 3 relationships were not significant, whereas 1 citation for abuse (citation F-225: 'Criminal screening investigating and reporting') was less likely, and another (citation F-226: 'Abuse prevention and policy development and implementation') was more likely to occur. For LPN/LVN and NA staff, no significant relationships were found. Another study ${ }^{33}$ reported that more RN and more LPN/LVN staff were associated with a decrease in the number of deficiency citations for restraints or side rails, whereas more NA staff was associated with an increase.

\section{Relationship Between Professional Staff Mix and QoC Outcomes}

Three studies ${ }^{13,26,28}$ examined the relationship between professional staff mix and QoC outcomes.

\section{Clinical Outcomes}

An association between a higher RN/total staff ratio ${ }^{28}$ and a higher RN/NA+LPN staff ratio ${ }^{13}$ and the prevalence of pressure ulcers could not be found. A higher RN/total staff ratio was associated with fewer urinary tract infections, ${ }^{28}$ whereas no significant relationship for mood decline ${ }^{24}$ was found. One study ${ }^{13}$ reported that a higher RN/NA+LPN ratio was associated with fewer reports of pain.

\section{Process-Related Outcomes}

One study ${ }^{13}$ examined the relationship between professional staff mix and processrelated resident outcomes. A higher RN/NA+LPN ratio was associated with a lower 
likelihood of catheterization, ${ }^{13}$ whereas for the use of physical restraints ${ }^{13}$ no significant relationship could be found.

\section{Administrative Outcomes}

One study ${ }^{26}$ examined the relationship between RN/total staff or RN/LVN ratios and the number of total and serious deficiencies (Table 2). For nursing homes meeting California's minimum standard for total nurse staffing level [3.2 or more total hours per resident day (HPRD)] during the 5-year period under consideration, a higher RN/total staff ratio was associated with fewer serious deficiencies, whereas a significant relationship with total deficiencies was not found. For nursing homes not meeting the state standard, a higher RN/total staff ratio was associated with fewer total deficiencies, but a significant relationship for serious deficiencies was not explored. For both nursing homes meeting and not meeting the state standard, a higher RN/LVN ratio was associated with fewer total as well as fewer serious deficiency citations. ${ }^{26}$ 
STAFFING AND QUALITY IN NURSING HOMES: A SYSTEMATIC REVIEW

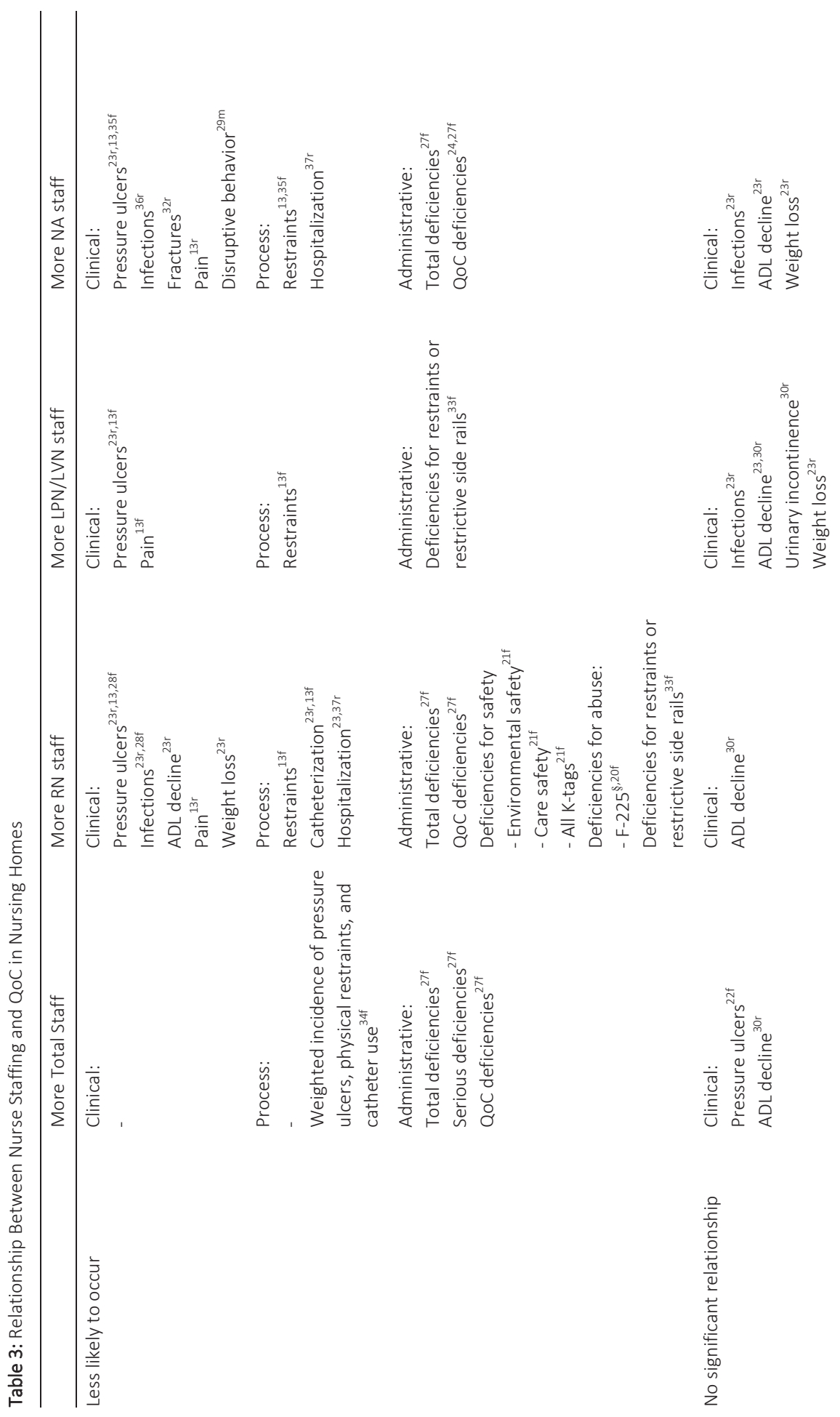




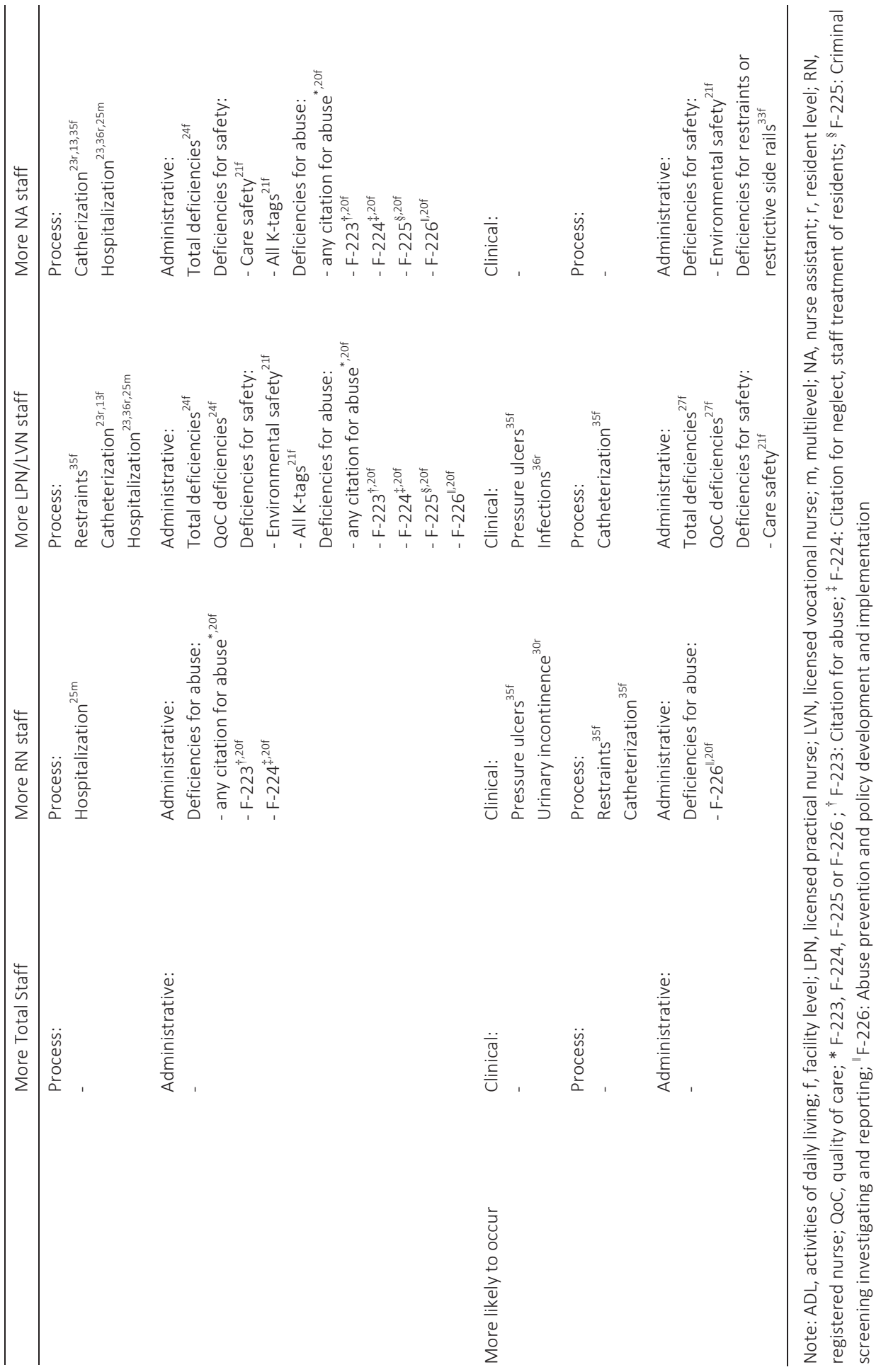




\section{DISCUSSION}

The aim of this review was to analyze findings from longitudinal studies examining the impact of nurse staffing on QoC outcomes in nursing homes. This review did not find convincing evidence for a positive relationship between nurse staffing levels and QoC. We found that higher numbers of nurse staff were associated with better as well as lower QoC outcomes. For example, for restraint use and catheterization, both positive (ie, less restraints/catheterization) and negative outcomes (ie, more restraints/catheterization) were found. With regard to pressure ulcers, we found that more staff led to better results, no matter who (RN, LPN/LVN or NA) delivered care.

This review is the first to provide an extensive overview of longitudinal studies examining the relationship between staffing and QoC outcomes in nursing homes. However, some limitations must be considered. We defined longitudinal studies as those studies repeatedly measuring QoC outcomes (dependent variable) over a period of time, and at least 1 measurement of staffing should precede the assessment of QoC. Ideally, the measurement of staffing characteristics should be measured at baseline and should precede the assessment of QoC outcome parameters. As in some included studies, the timing of measuring staffing characteristics was not clearly described; it is questionable whether authors are examining the effects of staffing on QoC. For nursing homes with high turnover rates, baseline staffing data might be less relevant. Although staffing was not measured at baseline in each study, the measurement of staffing at least preceded the follow-up assessment(s) of QoC. Furthermore, conducting a metaanalysis might have resulted in a more precise estimate of the relationship between nurse staffing and QoC. However, because of the heterogeneous nurse staffing and QoC data considered in the included studies, this analysis was not feasible. Finally, scales for assessing the quality of observational studies are diverse, emphasizing different aspects of methodological quality. We chose the Newcastle-Ottawa scale ${ }^{16}$ based on the recommendations of the Cochrane collaboration. ${ }^{38}$ Another scale could have resulted in a different quality assessment of studies, although it is unlikely that this would have resulted in the in-/exclusion of other studies.

Our review found no consistent evidence for a positive relationship between nurse

staffing and QoC, which is different from conclusions drawn in previous reviews. ${ }^{6,10,11}$ Our less positive and unexpected result can partly be explained by the fact that we included only longitudinal studies. In general, cross-sectional study designs, as included in the previous reviews, lead more often to positive findings. The different length of follow-up among the various studies is another methodological issue that might account for the heterogeneity of results. However, several theoretical drawbacks should be taken into account as well. 
First, most studies hypothesized a linear relationship between nurse staffing and QoC outcomes, assuming that more staff would lead to better QoC outcomes. As the relationship is probably nonlinear, meaning that a 1-unit increase in nursing staff will not necessarily lead to a 1-unit increase in $\mathrm{QoC}$, this could be an explanation for the lack of effect.

A second explanation for lack of effect might be the selection of QoC outcomes in the included studies. As each QoC outcome only serves as a proxy for overall QoC, ${ }^{10}$ QoC outcomes should be selected carefully. Some QoC outcomes could potentially be more nursing sensitive than others, leading to less nonsignificant findings. In addition, different content aspects regarding QoC hindered the interpretation of findings. For example, 4 studies ${ }^{23,25,36,37}$ used hospitalization as a proxy for poor overall health. However, only unnecessary hospitalizations should be taken into consideration as a proxy for poor QoC. ${ }^{39}$ In addition, in some studies assessing pressure ulcers, infections, or urinary incontinence, the authors did not distinguish between nosocomial and nonnosocomial outcomes. Only nosocomial outcomes, that is, outcomes developed during residents' stay in the nursing home, could be categorized as a nursing home QoC outcome. Moreover, the included studies do not examine the specific tasks executed by RN, LPN/LVN, and NA staff, and, therefore, these tasks were not known. It is questionable whether nursing staff of varying levels execute comparable tasks in different nursing homes. For example for pressure ulcers, we found that more staff led to better results, no matter who (RN, LPN/LVN, or NA) delivered care, raising questions about the contribution of each staffing category. RN staff could for example fulfill a leadership role in nursing homes, serving as role models for other nursing staff, positively influencing QoC. NA staff, in general, spends more time on direct resident care. Therefore, it could be that the higher frequency of resident contacts positively influences QoC.

Further research is needed before firm conclusions can be drawn. To deepen our insight into the impact of nurse staffing on QoC in nursing homes, future studies should consider characteristics beyond those of quantity of care provided. ${ }^{6}$ Two important characteristics are turnover and the use of agency staff. Recent studies suggest that higher turnover rates ${ }^{13,36}$ as well as more agency staff use ${ }^{13}$ can be associated with poorer QoC. Other examples of important variables related to QoC in nursing homes are nurses' professional competence, work experience, and adherence to procedures and guidelines. In addition, it would be useful to know the specific tasks executed by various nursing staff, with special attention paid to nurse leadership. As the number of less educated staff in nursing homes is high, a supervisory role of RNs in nursing homes might be crucial for the improvement of QoC. ${ }^{40}$ To obtain better insight into the total volume as well as the QoC provided to nursing home residents, future research needs to factor in the physician contribution of care as well. Besides assessing the quantity of care provided by physicians, it would be useful to also assess their specific tasks and supervisory role. In most countries, primary care practitioners deliver physician care in 
nursing homes, often with consultation from geriatricians. However, in The Netherlands, for example, nursing home medical specialists are available to provide medical care in nursing homes. Therefore, between countries, the influence of physicians on QoC in nursing homes may vary greatly. Recent evidence ${ }^{41}$ suggests that linking physicians to nursing homes through contracts and direct hire resulted in fewer rehospitalizations. However, only little is known about the relationship between physician staffing and QoC in nursing homes yet.

Moreover, future researchers should carefully select and interpret QoC outcomes for examining the relationship between staffing and QoC. For example, only unnecessary hospitalizations or nosocomial diseases should be taken into consideration as a proxy for poor QoC. For the analysis of the relationship between nurse staffing and QoC, statistical methods assuming a nonlinear relationship between staffing and QoC should be considered. With regard to methodology, the proper timing of data collection is crucial. As the influence of staffing on QoC is tested, it is necessary to collect staffing data prior to QoC data and cover a proper time period, long enough for QoC outcomes to develop.

\section{CONCLUSIONS}

No consistent evidence was found for a positive relationship between staffing and QoC including the specific contribution of each staffing category. Higher numbers of nurse staff were associated with better as well as lower QoC outcomes. Although some positive indications were suggested, major weaknesses in study designs limit the interpretation of the results. More knowledge on the theoretical relationship between staffing and QoC in nursing homes is necessary. Also, as most studies are US-based, the applicability of results cannot be generalized to other countries. All in all, the findings of this study demonstrate the necessity for well-designed longitudinal studies examining the effect of nurse staffing on QoC in nursing homes. 


\section{REFERENCES}

1. Miller SC, Miller EA, Jung HY, et al. Nursing home organizational change: the "Culture Change" movement as viewed by long-term care specialists. Med Care Res Rev. 2010;67:65s-81s.

2. Castle NG, Ferguson JC. What is nursing home quality and how is it measured? Gerontologist. 2010;50:426-442.

3. Harrington C, Choiniere J, Goldmann M, et al. Nursing home staffing standards and staffing levels in six countries. J Nurs Scholarsh. 2012;44:88-98.

4. Alexander GL. An analysis of nursing home quality measures and staffing. Qual Manag Health Care. 2008;17:242-251.

5. Katz PR. An international perspective on long term care: focus on nursing homes. J Am Med Dir Assoc. 2011;12:487-492.e1.

6. Castle NG. Nursing home caregiver staffing levels and quality of care: a literature review. J App/ Gerontol. 2008;27:375-405.

7. Mueller C, Arling G, Kane R, et al. Nursing home staffing standards: their relationship to nurse staffing levels. Gerontologist. 2006;46:74-80.

8. Harrington $C$, Kovner C, Mezey $M$, et al. Experts recommend minimum nurse staffing standards for nursing facilities in the United States. Gerontologist. 2000;40:5-16.

9. Shin JH, Bae SH. Nurse staffing, quality of care, and quality of life in US nursing homes, 1996-2011: an integrative review. J Gerontol Nurs. 2012;38:46-53.

10. Bostick JE, Rantz MJ, Flesner MK, et al. Systematic review of studies of staffing and quality in nursing homes. J Am Med Dir Assoc. 2006; 7:366-376.

11. Spilsbury K, Hewitt C, Stirk L, et al. The relationship between nurse staffing and quality of care in nursing homes: a systematic review. Int J Nurs Stud. 2011;48:732-750.

12. Mark BA, Harless DW, McCue M, et al. A longitudinal examination of hospital registered nurse staffing and quality of care. Health Serv Res. 2004;39:279-300.

13. Castle NG, Anderson RA. Caregiver staffing in nursing homes and their influence on quality of care: using dynamic panel estimation methods. Med Care. 2011;49:545-552.

14. Harrington C, Zimmerman D, Karon SL, et al. Nursing home staffing and its relationship to deficiencies. J Gerontol B Psychol Sci Soc Sci. 2000;55:S278-287.

15. Castle NG, Longest BB. Administrative deficiency citations and quality of care in nursing homes. Health Serv Manage Res. 2006;19:144-152.

16. http://www.ohri.ca/programs/clinical_epidemiology/oxford.asp. Accessed on May 27, 2013.

17. Khan Z, Almeida DR, Rahim K, et al. 10-Year Framingham risk in patients with retinal vein occlusion: a systematic review and meta-analysis. Can J Ophthalmol. 2013;48:40-45.e1.

18. Alotaibi GS, Almodaimegh H, McMurtry MS, et al. Do women bleed more than men when prescribed novel oral anticoagulants for venous thromboembolism? A sex-based meta-analysis. Thromb Res. 2013;132:185-189.

19. Arling G, Kane RL, Mueller C, et al. Nursing effort and quality of care for nursing home residents. Gerontologist. 2007;47:672-682.

20. Castle N. Nursing home deficiency citations for abuse. J App/ Gerontol. 2011;30:719-743.

21. Castle NG, Wagner LM, Ferguson JC, et al. Nursing home deficiency citations for safety. J Aging Soc Policy. 2011;23:34-57.

22. Hickey EC, Young GJ, Parker VA, et al. The effects of changes in nursing home staffing on pressure ulcer rates. J Am Med Dir Assoc. 2005;6:50-53.

23. Horn SD, Buerhaus $P$, Bergstrom $N$, et al. RN staffing time and outcomes of long-stay nursing home residents: pressure ulcers and other adverse outcomes are less likely as RNs spend more time on direct patient care. Am J Nurs. 2005;105:58-71.

24. Hyer K, Thomas KS, Branch LG, et al. The influence of nurse staffing levels on quality of care in nursing homes. Gerontologist. 2011;51:610-616. 
25. Intrator O, Castle NG, Mor V. Facility characteristics associated with hospitalization of nursing home residents: results of a national study. Med Care. 1999;37:228-237.

26. Kim H, Harrington C, Greene WH. Registered nurse staffing mix and quality of care in nursing homes: a longitudinal analysis. Gerontologist. 2009;49:81-90.

27. Kim H, Kovner C, Harrington C, et al. A panel data analysis of the relationships of nursing home staffing levels and standards to regulatory deficiencies. J Gerontol B Psychol Sci Soc Sci. 2009;64:269-278.

28. Konetzka RT, Stearns SC, Park J. The staffing-outcomes relationship in nursing homes. Health Serv Res. 2008;43:1025-1042.

29. Ooi WL, Morris JN, Brandeis GH, et al. Nursing home characteristics and the development of pressure sores and disruptive behaviour. Age Ageing. 1999;28:45-52.

30. Porell F, Caro FG, Silva A, et al. A longitudinal analysis of nursing home outcomes. Health Serv Res. 1998;33:835-865.

31. Rohrer JE, Hogan AJ. Modeling the outcomes of nursing home care. Soc Sci Med 1987;24:219-223.

32. Spector W, Shaffer T, Potter DE, et al. Risk factors associated with the occurrence of fractures in U.S. nursing homes: resident and facility characteristics and prescription medications. J Am Geriatr Soc. 2007;55:327-333.

33. Wagner LM, McDonald SM, Castle NG. Nursing home deficiency citations for physical restraints and restrictive side rails. West J Nurs Res. 2013;35:546-565.

34. Wan TT, Zhang NJ, Unruh L. Predictors of resident outcome improvement in nursing homes. West J Nurs Res. 2006;28:974-993.

35. Zhang X, Grabowski DC. Nursing home staffing and quality under the nursing home reform act. Gerontologist. 2004;44:13-23.

36. Zimmerman S, Gruber-Baldini AL, Hebel JR, et al. Nursing home facility risk factors for infection and hospitalization: importance of registered nurse turnover, administration, and social factors. J Am Geriatr Soc. 2002;50:1987-1995.

37. Cherubini A, Eusebi P, Dell'Aquila G, et al. Predictors of hospitalization in Italian nursing home residents: the U.L.I.S.S.E. project. J Am Med Dir Assoc. 2012;13:84.e5-84.e10.

38. Higgins JPT, Green S (editors). Cochrane Handbook for Systematic Reviews of Interventions Version 5.1.0 [updated March 2011]. The Cochrane Collaboration, 2011. Available from www. Cochranehandbook.org.

39. Ouslander JG, Maslow K. Geriatrics and the triple aim: defining preventable hospitalizations in the longterm care population. J Am Geriatr Soc. 2012;60:2313-2318

40. Weech-Maldonado R, Meret-Hanke L, Neff MC, et al. Nurse staffing patterns and quality of care in nursing homes. Health Care Manage Rev. 2004;29:107-116.

41. Lima JC, Intrator O, Karuza J, et al. Nursing home medical staff organization and 30-day rehospitalizations. J Am Med Dir Assoc. 2012;13:552-557. 



\title{
CHAPTER 3
}

\author{
RELATIONSHIP BETWEEN THE \\ PRESENCE OF \\ BACCALAUREATE-EDUCATED \\ RNS AND QUALITY OF \\ CARE: A CROSS-SECTIONAL \\ STUDY IN DUTCH LONG- \\ TERM CARE FACILITIES
}

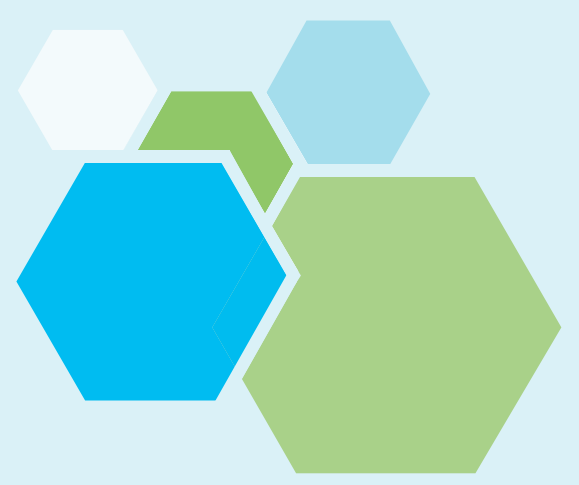

This chapter was published as:

Backhaus R, Van Rossum E, Verbeek H, Halfens RJG, Tan FES, Capezuti E, Hamers JPH.

Relationship Between the Presence of Baccalaureate-Educated RNs and Quality of Care : A Cross-Sectional Study in Dutch Long-Term Care Facilities. BMC Health Services Research 2017;17:53. 


\section{ABSTRACT}

Background: Recent evidence suggests that an increase in baccalaureate-educated registered nurses (BRNs) leads to better quality of care in hospitals. For geriatric longterm care facilities such as nursing homes, this relationship is less clear. Most studies assessing the relationship between nurse staffing and quality of care in long-term care facilities are US-based, and only a few have focused on the unique contribution of registered nurses. In this study, we focus on BRNs, as they are expected to serve as role models and change agents, while little is known about their unique contribution to quality of care in long-term care facilities.

Methods: We conducted a cross-sectional study among 282 wards and 6,145 residents from 95 Dutch long-term care facilities. The relationship between the presence of BRNs in wards and quality of care was assessed, controlling for background characteristics, i.e. ward size, and residents' age, gender, length of stay, comorbidities, and care dependency status. Multilevel logistic regression analyses, using a generalized estimating equation approach, were performed.

Results: $57 \%$ of the wards employed BRNs. In these wards, the BRNs delivered on average 4.8 minutes of care per resident per day. Among residents living in somatic wards that employed BRNs, the probability of experiencing a fall (odds ratio 1.44; 95\% $\mathrm{Cl}$ 1.06-1.96) and receiving antipsychotic drugs (odds ratio 2.15; 95\% $\mathrm{Cl} 1.66-2.78$ ) was higher, whereas the probability of having an indwelling urinary catheter was lower (odds ratio 0.70; 95\% $\mathrm{Cl}$ 0.53-0.91). Among residents living in psychogeriatric wards that employed BRNs, the probability of experiencing a medication incident was lower (odds ratio $0.68 ; 95 \% \mathrm{Cl}$ 0.49-0.95). For residents from both ward types, the probability of suffering from nosocomial pressure ulcers did not significantly differ for residents in wards employing BRNs.

Conclusions: In wards that employed BRNs, their mean amount of time spent per resident was low, while quality of care on most wards was acceptable. No consistent evidence was found for a relationship between the presence of BRNs in wards and quality of care outcomes, controlling for background characteristics. Future studies should consider the mediating and moderating role of staffing-related work processes and ward environment characteristics on quality of care. 


\section{BACKGROUND}

Recent evidence suggests that higher staffing levels and an increase in baccalaureateeducated registered nurses (BRNs) lead to better quality of care (QoC) in hospitals. ${ }^{1}$ For long-term care facilities (LTCFs) such as nursing homes, this relationship is less clear. ${ }^{2,3}$ It is assumed that an increase in BRNs could lead to an improvement in quality of life and QoC for LTCF residents as well. However, in most countries, the number of BRNs in LTCFs is low. ${ }^{4}$ Traditionally, working in LTCFs is associated with a low status career and inadequate salaries, ${ }^{5}$ reducing the chance to attract sufficient BRNs. When present BRNs currently often fulfill management positions. If involved in daily care, they frequently perform similar tasks as less educated staff. Their unique expertise could be used to serve as a role model, supervisor or innovator in the facility. As the number of less educated staff in LTCFs is high, BRNs can advance other staff practice to improve QoC. ${ }^{2,4,6}$ The importance of BRNs in LTCFs, and especially in nursing homes, is expected to increase further as new models of care will likely be implemented in the near future that require high level coordination and evaluation skills, ${ }^{7}$ and BRNs are expected to have more of these abstract thinking skills than less educated staff. ${ }^{8}$

International evidence for the added value of BRNs in LTCFs is scarce., ${ }^{2,3}$ Most studies assessing the relationship between nurse staffing and QoC in LTCFs are USbased, ${ }^{2,3,9}$ and only a few focus on the unique contribution of RNs. ${ }^{4,10}$ Most authors do not clarify the educational level of RNs, even though their educational backgrounds may differ substantially. ${ }^{11}$ This study focuses on the unique contribution of BRNs in LTCFs. The aim of this study was to examine the relationship between the presence of BRNs in wards and QoC in Dutch LTCFs. As a national database on staffing and QoC is lacking in the Netherlands, ${ }^{12}$ we conducted this study in cooperation with the Dutch Prevalence Measurement of Care Problems (LPZ: Landelijke Prevalentiemeting Zorgproblemen). ${ }^{13}$ The LPZ measurement is an annual, multicenter, cross-sectional point prevalence measurement of several care problems in LTCFs (such as pressure ulcers and fall incidents).

In this study, we focus on nurse sensitive indicators of QoC. The relationship between the presence of BRNs in wards and outcomes that are most sensitive to nursing care is addressed. We chose the following five outcomes from the LPZ database: nosocomial pressure ulcers, medication incidents, falls, antipsychotic drug use, and urinary indwelling catheter use. Pressure ulcers are the most frequently used QoC outcome for assessing the relationship between nurse staffing and QoC in LTCFs and seem to be a nurse-sensitive outcome., ${ }^{2,3}$ Ideally, only nosocomial pressure ulcers, which are pressure ulcers that developed during a resident's stay in the LTCF, should be considered.

In previous studies, higher nurse staffing levels in LTCFs were associated with a decrease in falls, ${ }^{14-17}$ but evidence on the relationship between better educated staff and the occurrence of falls in LTCFs is lacking. In addition, evidence is absent for a 
relationship between the presence of RNs in wards and medication incidents in LTCFs. However, we expect that medication incidents can be seen as a nurse-sensitive outcome as RNs spend much time on medication-related activities. ${ }^{18,19}$ Nevertheless, the occurrence of falls or medication incidents in LTCFs should be prevented as both can have serious consequences for residents, e.g. fall-related injuries or adverse drug events.

The prevalence rates of antipsychotic drug use in LTCFs are often high. ${ }^{20}$ We assume that the high prevalence rates can partly be explained by the inappropriate use of antipsychotic drugs, associated with poor QoC. ${ }^{10,20}$ Antipsychotic drug use is defined as inappropriate when a clinical rationale is absent such as a diagnosis of delirium, schizophrenia, or psychotic disorder. Recent studies suggest that the prescription of antipsychotics is not based on clinical reasons alone, but that direct care staff in nursing homes often believe that antipsychotics are the only treatment choice to manage challenging resident behaviors including screaming, moaning or wandering. ${ }^{20,21}$ The critical thinking skills of BRNs may place them in a better position to address challenging resident behavior without using antipsychotics, and might lead to less antipsychotic drug use on wards with higher BRN staffing levels.

Previous studies have considered fewer indwelling urinary catheters as a proxy for better urinary incontinence status of nursing home residents, $2,22,23$ and showed that more RN staff was associated with fewer catheterizations. ${ }^{22,23}$ The use of urinary indwelling catheters should be prevented as they can cause urinary tract infections, resident discomfort, and decreased mobility. ${ }^{10,24}$ BRNs are expected to have a better understanding of these negative consequences. Therefore, the prevalence rate of residents with indwelling urinary catheters might be lower on wards where BRNs are present.

\section{METHODS}

\section{Study design}

This study was conducted in cooperation with the Dutch LPZ cross-sectional point prevalence measurement in April 2014. Annually, the LPZ measurement takes place on the same day in different health care settings. Participation of health care organizations is voluntary. ${ }^{13}$ Data are collected at the organizational, ward, and resident level, using standardized questionnaires. Each participating organization appoints one coordinator who collects data at the organizational level, whereas ward managers provide data on their specific ward. Resident data (resident characteristics and prevalence of QoC outcomes) are collected by two health care professionals, one working on the resident's ward and one from another ward. ${ }^{13}$ Inter-rater reliability between observers was found to be good (Cohen's kappa 0.87). ${ }^{13,25,26}$ The standardized questionnaires are based on 
psychometrically tested instruments, existing guidelines or literature reviews, and are developed and regularly updated in collaboration with experts. ${ }^{27-34}$

To obtain BRN staffing data, we added 3 questions to the LPZ ward-level questionnaire. For each ward, the total number of hours of care delivered by BRNs was ascertained, as well as time spent in direct resident care (personal and nursing care, e.g. help with activities of daily living) and indirect care (e.g. staff education, coaching, and care innovation projects). No data were available on total nurse staffing.

\section{Setting and participants}

In Dutch LTCFs, most wards provide complex nursing care, whereas some wards provide only assistance with domestic tasks. ${ }^{35}$ Typically, long-term nursing care for older adults in the Netherlands is provided in somatic (for residents with physical disabilities) and psychogeriatric (for residents with dementia) wards. ${ }^{36}$ Therefore, we included only residents aged $>60$ years from psychogeriatric or somatic nursing care wards.

In the Netherlands, specifically trained nursing home medical specialists provide medical care for LTCF residents. ${ }^{36}$ Both these specialists as well as associated health professionals (e.g. psychologists, physiotherapists) are employed by the LTCF. Similar to other countries, the educational level of nursing staff varies. The largest proportion of nursing staff consist of certified nurse assistants (educational level 3) with 2-3 years of vocational training. ${ }^{36}$ Dutch certified nurse assistants are comparable to licensed practical/vocational nurses in the United States. ${ }^{37}$ There are also nurse assistants (educational level 2), nurse aides (educational level 1) as well as some uneducated staff. $^{38}$ In many LTCFs, the lowest percentage of staff are RNs (educational level 4) and BRNs (educational level 5).

In total, 282 wards and 6,145 residents from 95 LTCFs were included in our study. The 95 LTCFs are managed by 20 Dutch elderly care organizations.

\section{Data source, variables and measurement}

Table 1 presents the study variables and their measurement.

\section{Resident characteristics and QoC outcomes}

Residents' age, gender, length of stay, number of comorbidities, and care dependency status (CDS) $)^{39}$ were extracted from the LPZ, as well as the following QoC outcomes that were dichotomized (yes/no): nosocomial pressure ulcers, falls, antipsychotic drugs, medication incidents, and urinary indwelling catheters. 
Table 1: Study variables and their measurement

\begin{tabular}{ll}
\hline Variable & Measurement \\
\hline Resident characteristics & \\
Age & Man/woman \\
Length of stay & Age in years \\
Comorbidities & Number of days \\
& Number of comorbidities ( $\left.\underline{0}-24^{*}\right)$ : \\
& Infectious illness; cancer; endocrine, nutritional or metabolic illness/disease; \\
& diabetes mellitus; disease of blood or blood related organs; psychological \\
& disorders; dementia; nervous system disorder (excluding cerebrovascular \\
& accident (CVA)); spinal cord lesion/paraplegia; cardio vascular disease; \\
& CVA/hemiparesis; respiratory disorder/diseases, including nose and tonsils; \\
& disorder/disease of the digestive tract, including intestinal obstruction, \\
& peritonitis, hernia, liver, gallbladder, pancreas; disorder/disease of \\
& kidney/urinary tract, sexual organs; skin disorder/disease; motor \\
& disorder/disease; congenital disorders; injury resulting from accident(s), \\
& undesirable consequences of accident(s); symptoms and abnormal clinical or \\
lab findings, not elsewhere classified; overdose/substance abuse/addiction; & disease of the eye; disease of the ear; pregnancy, child birth; external factors \\
for disease
\end{tabular}

Care dependency

Presence of BRN

Presence of BRN

Quality of care outcomes

Nosocomial pressure ulcers

Medication incidents

Falls

Antipsychotic drug use

Indwelling urinary catheter use
Care Dependency Scale ${ }^{39}$ :

For each of the following 15 activities, the degree to which the resident is dependent upon care provided by others is indicated on a 5-point scale (completely dependent (1) - completely independent (5)*): eating and drinking, incontinence, body posture, mobility, day/night pattern, getting dressed and undressed, body temperature, hygiene, avoiding danger, communication, contact with others, sense of rules and values, daily activities, recreational activities, learning ability. ${ }^{13}$ For each resident, the total score (sum of 15 items) was divided by 15 to obtain a mean score.

At least one BRN present in ward

Resident suffers from at least one nosocomial pressure ulcer category 2-4 (European Pressure Ulcer Advisory Panel (EPUAP) \& National Pressure Ulcer Advisory Panel (NPUAP)) $)^{13,40}$ :

Category 2: Partial thickness

Category 3: Full thickness skin loss

Category 4: Full thickness tissue loss

Resident had at least one medication incident during the last 30 days **: Omitted dose

Wrong dose

Wrong time taken

Wrong drug

Wrong drug administration

Resident has fallen at least once during last 30 days **

Antipsychotic drug use during last 7 days **

Resident has an indwelling urinary catheter in place at the time 


\begin{tabular}{ll}
\hline Variable & Measurement \\
\hline Ward characteristics & \\
Ward type & Psychogeriatric/somatic nursing care ward \\
Ward size & Number of residents living on ward \\
\hline
\end{tabular}

Note: * underlined score is the most favorable score; ** answered by resident or responsible nurse and/or indicated in resident file $\mathrm{e}^{13}$

\section{Presence of $B R N$}

The total hours of care delivered by BRNs, as well as their hours spent on direct resident care and indirect care practices, were extracted from the LPZ. This data was used to distinguish between wards with at least one BRN present and wards that did not employ BRNs.

\section{Ward characteristics}

The ward type (somatic or psychogeriatric) as well as the ward size (number of residents living on ward) were extracted from the LPZ.

\section{Statistical analyses}

Data were analyzed with SPSS for Windows (version 22).

\section{Missing data}

In the Dutch LPZ, each participating organization can decide which QoC outcomes are assessed on the resident level within the organization. ${ }^{30}$ Therefore, because of nonparticipation, data on QoC outcomes were partly missing. In addition, for some residents, data collectors were not able to determine whether or not the resident suffered from a QoC problem, leading to missing data as well. The latter was the case for nosocomial pressure ulcers ( $n=22 ; 0.4 \%)$, falls $(n=53 ; 0.9 \%)$ and antipsychotic drug use $(n=28 ; 0.5 \%)$.

In total, among residents living in somatic wards, between $1.5 \%$ (falls) and $18.2 \%$ (nosocomial pressure ulcers) of data were missing. Among residents living in psychogeriatric wards, the amount of missing data ranged from $0.4 \%$ (falls) to $12.8 \%$ (nosocomial pressure ulcers). We cannot ignore these missing observations since the reasons for not including these by some organizations is not known. Therefore, three different approaches were taken to handle missing data. First, we performed a complete case analysis, ignoring missing data. Second, a sensitivity analysis was performed, in which all cases with missing data on a dependent variable were considered as "not suffering from the disease" (e.g., not having nosocomial pressure ulcers). Third, missing data were imputed, using multiple imputation techniques. To ensure the variability of predictors, ${ }^{41}$ the imputations were based on 7 (categorical) variables from the data set (BRNs working on ward, ward size, as well as residents' 
length of stay, age, gender, number of comorbidities, and care dependency). After performing the sensitivity analyses and the multiple imputations, the findings of these analyses were compared with those from the complete-case analyses.

\section{Univariate descriptive statistics}

Univariate descriptive statistics were computed. Means and standard deviations were calculated for resident characteristics and BRN staffing. For QoC outcomes, percentages of residents suffering from the outcome were calculated (frequency distribution).

(Multilevel) logistic regression analyses

For each QoC outcome, we estimated the relationship between the presence of BRNs in wards and QoC controlling for background characteristics, i.e. ward size, and residents' age, gender, length of stay, number of comorbidities, and care dependency status.

As the average time spent by BRNs per resident was low, we chose to dichotomize the BRN staffing variable, i.e. BRN not working on ward and BRN working on ward. Five control variables were recoded into categorical variables to avoid sparse cells and for the ease of interpretation. ${ }^{42}$ Ward size was recoded into 4 categories, i.e. fewer than 12 residents, 13-24 residents, 25-36 residents, more than 37 residents. Age was recoded into 4 categories, i.e. age 61-70, age 71-80, age 81-90, and age 91-110. Length of stay was recoded into 6 categories, i.e. 0-1 years, 1-2 years, 2-3 years, 3-4 years, 4-5 years, and longer than 5 years. The number of comorbidities was recoded into 5 categories: 1 comorbidity, 2 comorbidities, 3 comorbidities, 4 comorbidities, and 5 or more comorbidities. The total CDS score of each resident was changed into 1 of 5 categories (completely dependent (1) - completely independent (5)).

Due to differences in the care provided in somatic and psychogeriatric wards, separate analyses were performed among residents living in somatic and psychogeriatric wards. Ideally, to take into account possible correlations between residents living in the same ward and/or LTCF, 3-level logistic regression analyses should have been conducted in which residents were nested in wards and wards were nested in LTCFs. However, as some LTCFs were included with only one ward, it was not possible to conduct 3-level analyses examining the possible impact of wards and LTCFs simultaneously. These analyses led to estimation problems. Alternatively, two different 2-level logistic regression analyses were performed using a generalized estimating equation (GEE) approach. In these multilevel analyses, residents (level 1) were nested in wards (level 2) or residents (level 1) were nested in LTCFs (level 2). To test the correlation within residents living in the same ward or in the same LTCF, the intraclass correlation coefficient (ICC) was considered. Additionally, for each QoC outcome, a general logistic regression analysis was conducted for the resident level, not taking into account any hierarchy of data. 


\section{Ethical considerations}

All data were extracted from an existing database (LPZ), in which we received permission to conduct secondary analyses. The LPZ received ethical approval from the Medical Ethics Review Committee (METC) of the University Hospital Maastricht and Maastricht University.

\section{RESULTS}

Univariate descriptive statistics

Ward and resident characteristics

From the 282 participating wards, 117 were somatic wards (2,604 residents) and 165 were psychogeriatric wards (3,541 residents). Resident's mean age was 84 years (SD \pm 8 ) and $73 \%$ of the residents were female. Their mean length of stay was 2.9 years (1057 days $(S D \pm 1055)$ ), and on average, residents had 3 comorbidities (SD \pm 1 ). The mean CDS was 2.4 (SD \pm 1.2), meaning that, on average, residents were functionally dependent.

\section{Presence of $B R N$}

$57 \%$ of the wards employed a BRN, who delivered, on average, 4.8 minutes of care per resident per day (0.08 NHPRD, SD \pm 0.08$)$. The BRN conducted direct care practices on $91 \%$ of the wards that employed a BRN, and indirect care practices on $80 \%$ of the wards. On wards where the BRN had direct care practices, the average time spent on these practices was 3.6 minutes per resident per day (0.06 NHPRD, SD \pm 0.07$)$. On wards where the BRN had indirect care practices, the average time spent on these practices was 1.2 minutes per resident per day (0.02 NHPRD, SD \pm 0.02$)$.

QoC

From the residents that participated in our study, on average, $2.6 \%$ suffered from nosocomial pressure ulcers (category 2-4), 10.4\% had experienced a fall, and $5.3 \%$ a medication incident. $7.2 \%$ of the residents had an indwelling urinary catheter and $19.6 \%$ received antipsychotic drugs. Table 2 shows a considerable variation in prevalence rates among residents between somatic (more likely to have a nosocomial pressure ulcer, medication incident or indwelling urinary catheter) and psychogeriatric wards (more likely to fall or use antipsychotic drugs). When analyzing the relationship between the presence of BRNs in wards and nosocomial pressure ulcers among residents living on psychogeriatric wards, residents who were completely independent (i.e., CDS 5; n=92) were excluded, as none of these residents suffered from nosocomial pressure ulcers. 
Table 2: Differences in resident characteristics and prevalence rates of quality of care outcomes among residents living in somatic and psychogeriatric wards

\begin{tabular}{|c|c|c|}
\hline & $\begin{array}{l}\text { Residents living in somatic } \\
\text { wards } \\
(n=2,604)\end{array}$ & $\begin{array}{l}\text { Residents living in } \\
\text { psychogeriatric wards } \\
(n=3,541)\end{array}$ \\
\hline \multicolumn{3}{|l|}{ Resident characteristics } \\
\hline Age in years (mean, SD) * & $83 \pm 9$ & $84 \pm 7$ \\
\hline Female (\%) * & 70 & 75 \\
\hline Length of stay in years and days (mean, SD) * & $3.1(1,132 \pm 1,200)$ & $2.7(1,002 \pm 930)$ \\
\hline Number of comorbidities (mean, SD) * & $3 \pm 1$ & $3 \pm 1$ \\
\hline Care dependency (mean, SD) ${ }^{a_{1} *}$ & $2.9 \pm 1.2$ & $2.1 \pm 1.1$ \\
\hline \multicolumn{3}{|l|}{ Quality of care outcomes } \\
\hline Nosocomial pressure ulcers (\%) * & $3.4(n=2,131)$ & $1.9(n=3,086)$ \\
\hline Medication incidents (\%) * & $6.2(n=2,307)$ & $4.6(n=3,451)$ \\
\hline Falls $(\%) *$ & $7.6(n=2,564)$ & $12.4(n=3,528)$ \\
\hline Antipsychotic drug use (\%) * & $15.2(n=2,296)$ & $22.6(n=3,434)$ \\
\hline Indwelling urinary catheter use (\%) * & $11.7(n=2,271)$ & $3.9(n=3,143)$ \\
\hline
\end{tabular}

Note: SD = standard deviation; ${ }^{a}$ degree to which the resident is dependent upon care provided by others is indicated on a 5-point scale (completely dependent (1) - completely independent (5)); * significantly different among residents living in somatic and psychogeriatric wards $(p<0.01)$; independent samples t-test or chi-square)

\section{(Multilevel) logistic regression analyses}

For each QoC outcome, the results of the multilevel and the general logistic regression analyses were almost identical, and the ICC was low. In addition, the results of complete case analyses and those from the sensitivity analyses, as well as the analyses with imputed data were almost identical. Therefore, we present only the results of the general logistic regression analyses for complete cases (Table 3).

As indicated in Table 3, among residents living in somatic wards that employed BRNs, the probability of experiencing a fall (odds ratio $1.44 ; 95 \% \mathrm{Cl} 1.06-1.96$ ) and receiving antipsychotic drugs (odds ratio 2.15; $95 \% \mathrm{Cl} 1.66-2.78$ ) was higher, whereas the probability of having an indwelling urinary catheter was lower (odds ratio 0.70; 95\% $\mathrm{Cl}$ 0.53-0.91). Among residents living in psychogeriatric wards that employed BRNs, the probability of experiencing a medication incident was lower (odds ratio $0.68 ; 95 \% \mathrm{Cl}$ 0.49-0.95). For residents from both ward types, the probability of suffering from nosocomial pressure ulcers did not significantly differ for residents living in a ward that employed BRNs. In addition, among residents living in somatic wards, the probability of experiencing a medication incident did not significantly differ for residents living in a ward that employed BRNs. Among residents living in psychogeriatric wards, the probability of experiencing a fall, receiving antipsychotic drugs, or having an indwelling 
urinary catheter did not significantly differ for residents living in a ward that employed BRNs.

Table 3: Associations between presence of BRNs and quality of care indicators*

\begin{tabular}{|c|c|c|c|c|}
\hline Outcome measure & Ward type & $\begin{array}{l}\text { OR (BRN on } \\
\text { ward vs. no } \\
\text { BRN on ward) }\end{array}$ & $95 \% \mathrm{Cl}$ & $p$-value \\
\hline \multicolumn{5}{|l|}{ Nosocomial pressure ulcers } \\
\hline & Somatic & 0.68 & $0.42-1.10$ & 0.12 \\
\hline & Psychogeriatric & 0.79 & $0.46-1.38$ & 0.41 \\
\hline \multicolumn{5}{|l|}{ Medication incidents } \\
\hline & Somatic & 1.17 & $0.82-1.67$ & 0.39 \\
\hline & Psychogeriatric & 0.68 & $0.49-0.95$ & 0.02 \\
\hline \multicolumn{5}{|l|}{ Falls } \\
\hline & Somatic & 1.44 & $1.06-1.96$ & 0.02 \\
\hline & Psychogeriatric & 1.10 & $0.89-1.36$ & 0.38 \\
\hline \multicolumn{5}{|l|}{ Antipsychotic drug use } \\
\hline & Somatic & 2.15 & $1.66-2.78$ & 0.00 \\
\hline & Psychogeriatric & 1.06 & $0.89-1.26$ & 0.51 \\
\hline \multicolumn{5}{|c|}{ Urinary indwelling catheter use } \\
\hline & Somatic & 0.70 & $0.53-0.91$ & 0.01 \\
\hline & Psychogeriatric & 0.96 & $0.64-1.43$ & 0.83 \\
\hline
\end{tabular}

Note: * Fully adjusted models estimating the relationship between the presence of BRNs and quality of care controlling for background characteristics, i.e. ward size, and residents' age, gender, length of stay, amount of comorbidities, and care dependency status.

BRNs = baccalaureate-educated registered nurses $; O R=$ odds ratio $; 95 \% \mathrm{Cl}=95 \%$ confidence interval around OR

\section{DISCUSSION AND CONCLUSIONS}

In our study, there was no consistent relationship found between the presence of BRNs in wards and several QoC indicators, controlling for background characteristics. Among residents living in somatic wards that employed BRNs, an increased probability of experiencing a fall and receiving antipsychotic drugs was found, and a decreased probability of having an indwelling urinary catheter. No significant differences were detected for nosocomial pressure ulcers and medication incidents. For residents living in psychogeriatric wards that employed BRNs a decreased probability of experiencing a medication incident was found, whereas the probability for developing any of the other QoC outcomes did not significantly differ.

Two systematic reviews also reported inconsistent findings on QoC indicators. ${ }^{2,3}$ For this study, there are several factors that need to be taken into consideration. First, only 
$57 \%$ of the wards employed a BRN, who delivered, on average, 4.8 minutes of care per resident per day. BRN staffing levels may not have been high enough to establish better QoC outcomes. For comparison, in a recent Swiss study among 402 wards from 155 nursing homes, on average $32 \%$ of all full-time equivalents (FTEs) per ward were RNs. ${ }^{43}$ In a recent US study among nursing homes in Colorado, ${ }^{10}$ RNs spent on average 36 minutes of care per resident per day. As with all other studies examining the relationship between $\mathrm{RN}$ staffing and QoC, both studies did not indicate the educational background of RNs.

Second, for residents living in both types of wards, the prevalence of QoC problems seems low compared to studies conducted in other countries. However, differences in operationalization and measurement methods have to be considered when comparing prevalence rates to other studies, ${ }^{44}$ making comparisons difficult. ${ }^{26}$ The prevalence of nosocomial pressure ulcers was especially low, which may explain why the probability of suffering from nosocomial pressure ulcers did not significantly differ among residents living in wards that did or did not employ BRNs. For both ward types, antipsychotic drug use was the most prevalent QoC problem, yet the prevalence rate of $19.6 \%$ was low compared to prevalence rates in other countries. For example, in a study among Belgian nursing home residents the prevalence rate was 32.9\%. ${ }^{45}$ Nevertheless, the fact that, in our sample, one resident out of every five was provided with antipsychotic drugs, could be a signal of inappropriate drug use. Only unnecessary antipsychotic drug use should be considered as poor QoC. In this study, we were not able to distinguish between (in)appropriate antipsychotic drug use.

Third, the practices of BRNs working in Dutch LTCFs may not differ from those conducted by other nursing staff, meaning that BRNs are not employed optimally to benefit from their unique contribution to QoC outcomes. It seems that most BRNs are responsible for multiple wards, which is reflected in the low amount of time spent per resident per day. BRNs might only see residents that are in acute, complex care situations (e.g., when a decision whether or not to hospitalize the resident has to be made), instead of looking at each resident's overall care plan.

The findings of this study should be interpreted carefully. The cross-sectional design provides no information about causality. For example, we cannot say whether the employment of BRNs in somatic wards led to an increased probability of receiving antipsychotic drugs or whether BRNs were employed due to high antipsychotic drug use. As some LTCFs were included with only one ward, it was not possible to conduct 3level analyses examining the possible impact of wards and LTCFs simultaneously. Moreover, we had to focus on BRNs alone, not taking into consideration the contribution of other nursing staff, nursing home medical specialists and allied professionals working in Dutch LTCFs. In addition, due the low average amount of time BRNs spent on wards, we could only distinguish between wards that did or did not employ BRNs, not taking into consideration the actual amount of time BRNs worked on the wards. To compare BRN staffing among wards, we calculated NHPRDs. However, 
BRNs may only deliver care to residents with the most complex care problems. In our analyses, we distinguished between residents living in somatic and psychogeriatric wards, while in practice, the difference may not be that clear-cut, e.g., some residents living in somatic wards may suffer from dementia or residents living in psychogeriatric wards from somatic diseases as well. Finally, our analyses were limited to the QoC outcomes measured in the LPZ, while BRNs may influence other outcomes, e.g., outcomes related to quality of life of residents. Despite these limitations, our study is the first that provides insight into the relationship between the presence of BRNs in wards and QoC for Dutch LTCFs. As we made use of an existing data infrastructure (LPZ), the sample size was large $(6,145$ residents), and collected data was of good quality.

Although the Dutch government is making efforts to increase the number of BRNs working in elder care, the number of BRNs working in LTCFs is still low, as in $43 \%$ of the wards no BRNs were employed. Even for wards that employed BRNs, the mean amount of time spent per resident was low. For LTCFs it is therefore important to carefully think about how to best allocate BRNs on their wards. In recent years, there has been a call to shift emphasis back to the provision of essential nursing care, e.g., providing physical comfort and psychological support or establishing meaningful encounters between staff and residents. ${ }^{46,47}$ It might be the case that BRNs add particular value to improving essential nursing care, thus future studies should consider this. Recently, David Richards has posed the question whether nursing outcomes might need to be defined in terms of a concept called 'amalgamation of marginal gains'. 47,48 During a hospital visit Richards experienced that small, individual actions by nurses only had marginal impact on his well-being, while in total, all these 'small actions' significantly reduced his feelings of discomfort and anxiety. By focusing on isolated components of essential nursing care (e.g., communication), Richards stresses one may miss the 'power of amalgamation' ${ }^{47}$

In our study, we focused on the presence of BRNs in wards rather than considering staffing as a 'multidimensional construct'. ${ }^{49}$ Future studies should also consider the mediating and moderating role of staffing-related work processes and ward environment characteristics. For example, more BRNs in the mix of staff might lead to better teamwork and communication, that could result in better QoC. ${ }^{17,50}$ Other examples of work processes BRNs might have influence on are the coordination of care $^{51}$ and the collaboration between nursing staff and nursing home medical specialists or allied health professionals. ${ }^{52}$ In addition, BRNs might indirectly add value to QoC in LTCFs by acting as a clinical leader and coach for other nursing staff. ${ }^{53}$ Moreover, BRNs might also have an influence on ward environment characteristics like the organizational culture or the team climate, which were associated with better QoC in previous studies. ${ }^{54,55}$ Conducting mixed methods-studies, e.g. by combining direct observations with stakeholder interviews, may help to obtain more information on observable behavior (e.g., interactions with residents or other staff and other 'small actions') and unobservable cognitive work of BRNs leading to added value for residents, family members, and staff. ${ }^{53}$ 


\section{REFERENCES}

1. Aiken LH, Sloane DM, Bruyneel L, et al. Nurse staffing and education and hospital mortality in nine European countries: a retrospective observational study. Lancet. 2014;383:1824-1830.

2. Backhaus R, Verbeek H, Van Rossum E, Capezuti E, Hamers JPH. Nurse staffing impact on quality of care in nursing homes: a systematic review of longitudinal studies. J Am Med Dir Assoc. 2014;15:383-393.

3. Spilsbury K, Hewitt C, Stirk L, Bowman C: The relationship between nurse staffing and quality of care in nursing homes: A systematic review. Int J Nurs Stud. 2011;48:732-750.

4. Weech-Maldonado R, Meret-Hanke L, Neff MC, Mor V. Nurse staffing patterns and quality of care in nursing homes. Health Care Manage Rev. 2004;29:107-116.

5. Tolson D, Rolland Y, Andrieu S, et al. International association of gerontology and geriatrics: a global agenda for clinical research and quality of care in nursing homes. J Am Med Dir Assoc. 2011;12:184-189.

6. Boström AM, Rudman A, Ehrenberg A, Gustavsson J, Wallin L. Factors associated with evidence-based practice among registered nurses in Sweden: a national cross-sectional study. BMC Health Serv Res. 2013;13:165.

7. Auerbach DI, Staiger DO, Muench U, Buerhaus PI. The nursing workforce in an era of health care reform. N Engl J Med. 2013; 368:1470-1472.

8. Backhaus R, Verbeek H, Van Rossum E, Capezuti E, Hamers JPH. Future distinguishing competencies of baccalaureate-educated registered nurses in nursing homes. Geriatr Nurs. 2015;36:438-444.

9. Havig A, Skogstad A, Kjekshus L, Romoren T. Leadership, staffing and quality of care in nursing homes. BMC Health Serv Res. 2011;11:327.

10. Lee HY, Blegen MA, Harrington C. The effects of RN staffing hours on nursing home quality: A two-stage model. Int J Nurs Stud. 2014;51:409-417.

11. Hallberg IR, Cabrera E, Jolley D, et al. Professional care providers in dementia care in eight European countries; their training and involvement in early dementia stage and in home care. Dementia. 2016;15:931-957.

12. Onder G, Carpenter I, Finne-Soveri H, et al. Assessment of nursing home residents in Europe: the Services and Health for Elderly in Long TERm care (SHELTER) study. BMC Health Serv Res. 2012;12:5.

13. Van Nie-Visser NC, Schols JMGA, Meesterberends E, Lohrmann C, Meijers JMM, Halfens RJG. An international prevalence measurement of care problems: study protocol. J Adv Nurs. 2013;69:e18-e29.

14. Hofmann MT, Bankes PF, Javed A, Selhat M. Decreasing the incidence of falls in the nursing home in a cost-conscious environment: a pilot study. J Am Med Dir Assoc. 2003;4:95-97.

15. Leland NE, Gozalo P, Teno J, Mor V. Falls in newly admitted nursing home residents: a national study. J Am Geriatr Soc. 2012;60:939-945.

16. Sandoval Garrido FA, Tamiya N, Kashiwagi M, et al. Relationship between structural characteristics and outcome quality indicators at health care facilities for the elderly requiring long-term care in Japan from a nationwide survey. Geriatr Gerontol Int. 2014;14:301-308.

17. Schwendimann R, Zúñiga F, Ausserhofer D, Schubert M, Engberg S, De Geest S. Swiss Nursing Homes Human Resources Project (SHURP): protocol of an observational study. J Adv Nurs. 2014;70:915-926.

18. Biron AD, Lavoie-Tremblay M, Loiselle CG. Characteristics of work interruptions during medication administration. J Nurs Scholarsh. 2009;41:330-336.

19. Keohane $C A$, Bane AD, Featherstone $E$, et al. Quantifying nursing workflow in medication administration. J Nurs Adm. 2008;38:19-26.

20. Van der Putten MJ, Wetzels RB, Bor H, Zuidema SU, Koopmans RT. Antipsychotic drug prescription rates among Dutch nursing homes: the influence of patient characteristics and the dementia special care unit. Aging Ment Health. 2014;18:828-832.

21. Lemay CA, Mazor KM, Field TS, et al. Knowledge of and perceived need for evidence-based education about antipsychotic medications among nursing home leadership and staff. J Am Med Dir Assoc. 2013;14:895-900. 
22. Castle NG, Anderson RA. Caregiver staffing in nursing homes and their influence on quality of care: using dynamic panel estimation methods. Med Care. 2011;49:545-552.

23. Horn S, Buerhaus P, Bergstrom N, Smout R. RN staffing time and outcomes of long-stay nursing home residents: pressure ulcers and other adverse outcomes are less likely as RNs spend more time on direct patient care. Am J Nurs. 2005;105:58-70.

24. Fakih MG, Krein SL, Edson B, Watson SR, Battles JB, Saint S. Engaging health care workers to prevent catheter-associated urinary tract infection and avert patient harm. Am J Infect Control. 2014;42:S223S229.

25. Meijers JM, Candel MJ, Schols JM, Halfens RJ. Decreasing trends in malnutrition prevalence rates explained by regular audits and feedback. J Nutr. 2009;139:1381-1386.

26. Meijers JM, Schols JM, Van Bokhorst-de van der Schueren MA, Dassen T, Janssen MA, Halfens RJ. Malnutrition prevalence in the Netherlands: results of the annual Dutch national prevalence measurement of care problems. Br J Nutr. 2009;101:417-423.

27. Bours G, Halfens R, Lubbers M, Haalboom J. The development of a national registration form to measure the prevalence of pressure ulcers in the Netherlands. Ostomy Wound Manage. 1999;45:28-33.

28. Bours GJJW, Halfens RJG, Abu-Saad HH, Grol RTPM. Prevalence, prevention, and treatment of pressure ulcers: descriptive study in 89 institutions in the Netherlands. Res Nurs Health. 2002;25:99-110.

29. Dijkstra A, Buist G, Dassen T, Van den Heuvel W. Het meten van zorgafhankelijkheid met de ZorgAfhankelijkheidsSchaal, Care Dependency Scale (CDS): een handleiding. Groningen: Noordelijk Centrum voor Gezondheidsvraagstukken, Rijksuniversiteit Groningen; 1999.

30. Halfens RJG, Meesterberends E, Van Nie-Visser NC, et al. International prevalence measurement of care problems: results. J Adv Nurs. 2013;69:e5-e17.

31. Hannestad YS, Rortveit G, Sandvik H, Hunskaar S. A community-based epidemiological survey of female urinary incontinence:: the Norwegian EPINCONT Study. J Clin Epidemiol. 2000;53:1150-1157.

32. Meijers JMM, Van Bokhorst-de van der Schueren MAE, Schols JMGA, Soeters PB, Halfens RJG. Defining malnutrition: mission or mission impossible? Nutrition. 2010;26:432-440.

33. Neyens J, Dijcks B, Van Haastregt J, et al. The development of a multidisciplinary fall risk evaluation tool for demented nursing home patients in the Netherlands. BMC Public Health. 2006;6:74.

34. Thüroff JW, Abrams $P$, Andersson KE, et al. EAU Guidelines on Urinary Incontinence. Eur Urol. 2011;59:387-400.

35. De Meijer C, Koopmanschap M, D’ Uva TB, Van Doorslaer E. Determinants of long-term care spending: Age, time to death or disability? J Health Econ. 2011;30:425-438.

36. Huls M, De Rooij SE, Diepstraten A, Koopmans R, Helmich E. Learning to care for older patients: hospitals and nursing homes as learning environments. Med Educ. 2015;49:332-339.

37. Verkaik R, Francke AL, van Meijel B, Spreeuwenberg PMM, Ribbe MW, Bensing JM. The introduction of a nursing guideline on depression at psychogeriatric nursing home wards: effects on certified nurse assistants. Int J Nurs Stud. 2011;48:710-719.

38. Willemse B, Depla M, Smit D, Pot A. The relationship between small-scale nursing home care for people with dementia and staff's perceived job characteristics. Int Psychogeriatr. 2014;26:805-816.

39. Dijkstra A, Yont GH, Korhan EA, Muszalik M, Kedziora-Kornatowska K, Suzuki M. The care dependency scale for measuring basic human needs: an international comparison. J Adv Nurs. 2012;68:2341-2348.

40. European Pressure Ulcer Advisory Panel and National Pressure Ulcer Advisory Panel. Prevention and treatment of pressure ulcers: quick reference guide. Washington DC: National Pressure Ulcer Advisory Panel; 2009.

41. Sterne JAC, White IR, Carlin JB, et al. Multiple imputation for missing data in epidemiological and clinical research: potential and pitfalls. BMJ. 2009;338:b2393.

42. Zhang F, Lewis M, Yang G, Iriondo-Perez J, Zeng Y, Liu J. Apolipoprotein E polymorphism, life stress and self-reported health among older adults. J Epidemiol Community Health. 2008;62:e3.

43. Zúñiga F, Ausserhofer D, Hamers JPH, Engberg S, Simon M, Schwendimann R. Are staffing, work environment, work stressors, and rationing of care related to care workers' perception of quality of care? A cross-sectional study. J Am Med Dir Assoc. 2015;16:860-866. 
44. Schüssler S, Dassen T, Lohrmann C. Prevalence of care dependency and nursing care problems in nursing home residents with dementia: a literature review. International Journal of Caring Sciences. 2014;7:338352.

45. Azermai M, Elseviers M, Petrovic M, van Bortel L, Stichele RV. Assessment of antipsychotic prescribing in Belgian nursing homes. Int Psychogeriatr. 2011;23:1240-1248.

46. Kitson A, Conroy T, Kuluski K, Locock L, Lyons R. Reclaiming and redefining the fundamentals of care: nursing's response to meeting patients' basic human needs. Adelaide: School of Nursing, the University of Adelaide; 2013.

47. Richards DA. Complex interventions and the amalgamation of marginal gains: a way forward for understanding and researching essential nursing care? Int J Nurs Stud. 2015;52:1143-1145.

48. Cavendish M. Boy Racer. New York: Random House; 2010.

49. Arling G, Mueller C. Nurse staffing and quality: the unanswered question. J Am Med Dir Assoc. 2014;15:376-378.

50. Colon-Emeric CS, McConnell E, Pinheiro SO, et al. CONNECT for better fall prevention in nursing homes: results from a pilot intervention study. J Am Geriatr Soc. 2013;61:2150-2159.

51. Temkin-Greener $\mathrm{H}$, Zheng N, Katz P, Zhao H, Mukamel DB. Measuring work environment and performance in nursing homes. Med Care. 2009;47:482-491.

52. Van Bogaert P, Timmermans O, Weeks SM, van Heusden D, Wouters K, Franck E. Nursing unit teams matter: impact of unit-level nurse practice environment, nurse work characteristics, and burnout on nurse reported job outcomes, and quality of care, and patient adverse events - a cross-sectional survey. Int J Nurs Stud. 2014;51:1123-1134.

53. Dellefield ME, Castle NG, McGilton KS, Spilsbury K. The Relationship Between Registered Nurses and Nursing Home Quality: An Integrative Review (2008-2014). Nurs Econ. 2015;33:95.

54. Bower P, Campbell S, Bojke C, Sibbald B. Team structure, team climate and the quality of care in primary care: an observational study. Qual Saf Health Care. 2003;12:273-279.

55. Van Beek AP, Gerritsen DL. The relationship between organizational culture of nursing staff and quality of care for residents with dementia: questionnaire surveys and systematic observations in nursing homes. Int J Nurs Stud. 2010;47:1274-1282. 


\title{
CHAPTER 4
}

\author{
QUANTITY OF STAFF AND \\ QUALITY OF CARE IN DUTCH \\ NURSING HOMES: \\ A CROSS-SECTIONAL STUDY
}

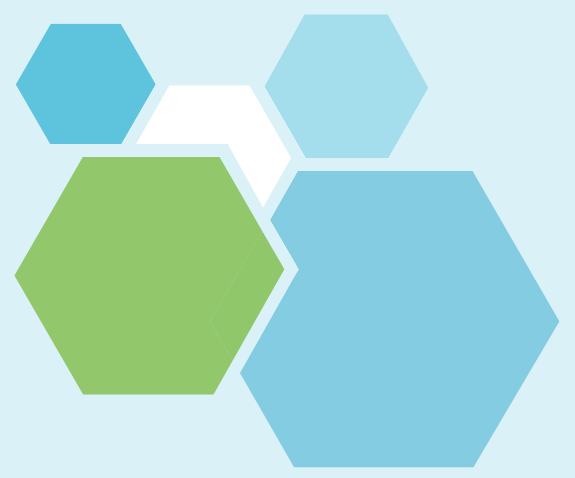

This chapter was published as:

Backhaus R, Van Rossum E, Verbeek H, Halfens RJG, Tan FES, Capezuti E, Hamers JPH. Quantity of Staff and Quality of Care in Dutch Nursing Homes : A Cross-Sectional Study. The Journal of Nursing Home Research Sciences 2016;2:90-93. 


\section{ABSTRACT}

The general belief is that the number of total staff hours per resident day (HPRD) and the staff mix (\% registered nurses/total staff) are associated with quality of care (QoC) in nursing homes. However, findings from studies examining these relationships are inconsistent. In this brief report, we present findings from a cross-sectional, observational study on the relationship between HPRD and clinical as well as staffreported QoC indicators. Data were collected in 55 nursing home wards that participated in the Dutch Prevalence Measurement of Care Problems in April 2014. We conducted adjusted (multilevel) logistic regression analyses for clinical outcomes and multilevel linear regression analyses for staff perception of QoC. Overall, we were unable to demonstrate a relationship between HPRD and QoC. Our findings underscore that focusing on quantity of nursing care might not improve QoC in nursing homes. The quality of the team should be taken into consideration as well. 


\section{INTRODUCTION}

In many European countries, concerns about nursing home quality have led to political discussions on increasing funding, as it is widely assumed that more resources are needed to improve quality of care $(\mathrm{Q} O \mathrm{C})$ in nursing homes. ${ }^{1} \mathrm{~A}$ general, persistent belief is that the number of total staff hours per resident day and the staff mix (\% registered nurses/total staff) are associated with QoC in nursing homes. Studies examining this relationship have mostly been conducted in the US and findings are inconsistent. ${ }^{2-5}$ Findings based on US data cannot be generalized to European countries, as educational backgrounds of nursing staff differ. For example, in the US, a minimum of 75 hours of initial training are required to become a certified nurse assistant, ${ }^{6}$ while in the Netherlands, the length of the educational program to become a nurse assistant is two years. First European studies show that evidence for a relationship between the number of total staff hours per resident day or the staff mix and QoC in nursing homes is lacking. ${ }^{7-10}$ Studies that find a positive relationship mostly analyzed facility-level data from large US databases (e.g., OSCAR (Online Survey, Certification and Reporting)) that are not primarily intended for research purposes. ${ }^{2,4}$ The accuracy of staffing data in such databases might be doubted, as they not necessarily reflect reality. ${ }^{5}$ Moreover, data on staffing and QoC in these databases do not necessarily cover the same time periods, meaning that researchers are analyzing non-contemporaneous data. ${ }^{4}$ In addition, in many studies, QoC is operationalized only as clinical resident outcomes, such as the prevalence of falls or nosocomial pressure ulcers. A limitation of these outcomes is that they only give an indication of QoC at one time point. As staffs' perception of QoC develops over time and is not based on isolated care components, ${ }^{7}$ it is an important addition to clinical outcomes. Staffs' perception of QoC has been used in evaluating care in other settings and is considered a valid proxy measure of QoC. ${ }^{7,11}$

Evidence on the relationship between quantity of staff or the staff mix and staffs' perception of QoC in nursing homes is lacking. In this brief report, we present data on the relationship between the number of staff hours per resident as well as the staff mix and QoC in Dutch nursing homes. We included clinical outcomes as well as staff perception of QoC to assess these relationships.

\section{METHODS}

A cross-sectional, observational study was conducted among 55 wards within 21 nursing homes that participated in the Dutch Prevalence Measurement of Care Problems (LPZ) in April 2014. The LPZ is a cross-sectional point prevalence measurement of several care problems (such as pressure ulcers and fall incidents), taking place annually on the same day in different health care settings. ${ }^{12}$ Unlike in some other countries, a national database on staffing and QoC is lacking in the Netherlands ${ }^{13}$ and participation in the LPZ is voluntary. Data collection takes place on one day at facility, ward, and resident level, using standardized questionnaires that are based on 
psychometrically tested instruments or existing guidelines and literature reviews. The questionnaires are developed and regularly updated in collaboration with expert groups. In this study, we only used data measured on resident level (resident characteristics and prevalence of clinical QoC outcomes). Data are collected according a standardized protocol. Within each ward, two health care professionals collected data on resident level. One professional was working on the residents' ward and one was from another ward. Inter-rater reliability between observers was tested to be good (Cohen's kappa 0.87). ${ }^{12,14,15}$ The Medical Ethics Review Committee of the University Hospital Maastricht and Maastricht University approved the study protocol (METC14-4057).

Resident characteristics (age, gender, length of stay, comorbidities, care dependency status) as well as clinical QoC outcomes (dichotomized (yes/no)) nosocomial pressure ulcers, falls, antipsychotic drug use, medication incidents, urinary indwelling catheters - were extracted from the LPZ database. To obtain insight into staff perception of QoC, at least five staff members from every ward were invited to assess the QoC on their ward. They graded the overall QoC on their ward (grade 1-10, higher score indicating better $\mathrm{QOC}$ ) and were asked to which extent they agreed with the statement "In case a family member had to be admitted to a nursing home now, I would recommend this ward" (completely not agree (1) - completely agree (5)).

Based on their ward roster, ward managers provided average total direct care staff hours for an average day within the last seven days. Based on the actual ward roster, they described the number of direct care staff members that worked on that day (morning (7:00 am - 12:00 noon), day (12:00 noon - 5:00 pm), evening (5:00 pm 11:00 pm), and night (11:00 pm - 7:00 am) shifts), each staff members' educational background and the exact start and finish time of their shift. Direct care staff consisted of (certified) nurse assistants, nurse aids, specially trained feeding assistants, trainees, untrained staff, and vocationally or baccalaureate-educated registered nurses (RNs). For each ward the total direct care staff hours per resident per day were calculated, further referred to as hours per resident per day (HPRD). In addition, the staff mix was calculated for each ward.

Separate analyses were performed for somatic and psychogeriatric wards due to differences in prevalence of clinical outcomes. We estimated the relationship between HPRD and QOC (dependent variable), adjusting for ward size and resident characteristics (i.e., age, gender, length of stay, number of comorbidities, and care dependency status). We conducted (multilevel) logistic regression analyses for clinical outcomes and multilevel linear regression analyses (random intercept) for staff perception of QoC. 


\section{RESULTS}

\section{Descriptive statistics are described in Table 1.}

Table 1: Differences in study variable characteristics among somatic and psychogeriatric wards

\begin{tabular}{|c|c|c|}
\hline & $\begin{array}{l}\text { Somatic wards } \\
\qquad(n=24)\end{array}$ & $\begin{array}{l}\text { Psychogeriatric wards } \\
\qquad(n=31)\end{array}$ \\
\hline \multicolumn{3}{|l|}{ Ward characteristics ( $n=55)$} \\
\hline Total direct care staffing (HPRD; mean, SD) & $3.06 \pm 0.58$ & $3.15 \pm 0.40$ \\
\hline - Baccalaureate-educated RN staffing (HPRD; mean, SD) & $0.02 \pm 0.03$ & $0.02 \pm 0.05$ \\
\hline - Vocationally trained RN staffing (HPRD; mean, SD) & $0.16 \pm 0.21$ & $0.15 \pm 0.15$ \\
\hline - Certified nurse assistant staffing (HPRD; mean, SD) & $1.91 \pm 0.34$ & $1.77 \pm 0.38$ \\
\hline - Nurse assistant staffing (HPRD; mean, SD) & $0.41 \pm 0.43$ & $0.64 \pm 0.38$ \\
\hline - Nurse aide staffing (HPRD; mean, SD) & $0.08 \pm 0.17$ & $0.12 \pm 0.25$ \\
\hline Staff mix (\% RN/total staff; mean, SD) & $0.05 \pm 0.06$ & $0.05 \pm 0.05$ \\
\hline Ward size (mean, SD) & $27 \pm 8$ & $27 \pm 10$ \\
\hline Resident characteristics & 617 residents & 821 residents \\
\hline Age in years (mean, SD) & $79 \pm 11$ & $83 \pm 9$ \\
\hline Female (\%) & 65 & 72 \\
\hline Length of stay in years and days (mean, SD) & $2.7(1,002 \pm 1,225)$ & $2.6(959 \pm 873)$ \\
\hline Number of comorbidities (mean, SD) & $3 \pm 1$ & $3 \pm 1$ \\
\hline Care dependency (scale range: $1-5$; mean, SD) & $2.7 \pm 1.1$ & $2.1 \pm 1.1$ \\
\hline Staff characteristics & 104 staff members & 153 staff members \\
\hline Number of work hours per week (mean, SD) & $29.45 \pm 5.17$ & $29.18 \pm 5.48$ \\
\hline \multicolumn{3}{|l|}{ Educational background } \\
\hline - Baccalaureate-educated RN (\%) & $2.9(n=3)$ & $2.0(n=3)$ \\
\hline - Vocationally trained RN (\%) & $18.3(n=19)$ & $10.5(n=16)$ \\
\hline - Certified nurse assistant (\%) & $78.8(n=82)$ & $87.6(n=134)$ \\
\hline \multicolumn{3}{|l|}{ Quality of care outcomes } \\
\hline Clinical outcomes $^{\dagger}$ & 617 residents & 821 residents \\
\hline Nosocomial pressure ulcers (\% residents) ${ }^{\ddagger}$ & $5.2(n=597)$ & $2.4(n=803)$ \\
\hline Medication incidents (\% residents) & $7.1(n=562)$ & $4.7(n=790)$ \\
\hline Falls (\% residents) ${ }^{\ddagger}$ & $6.1(n=604)$ & $12.0(n=817)$ \\
\hline Antipsychotic drug use (\% residents) ${ }^{\ddagger}$ & $10.7(n=561)$ & $26.6(n=790)$ \\
\hline Indwelling urinary catheter use (\% residents) ${ }^{\ddagger}$ & $17.0(n=601)$ & $4.0(n=806)$ \\
\hline \multicolumn{3}{|l|}{ Staff-reported outcomes } \\
\hline Grade overall quality of care (scale range: $1-\underline{10}$; mean, SD) $)^{\ddagger \S}$ & $7.4 \pm 0.9$ & $7.7 \pm 0.7$ \\
\hline Recommending the ward (scale range: $1-5 ;$ mean, SD) ${ }^{\ddagger \S}$ & $3.5 \pm 1.0$ & $3.9 \pm 0.9$ \\
\hline
\end{tabular}

Note: SD = standard deviation; * degree to which the resident is dependent upon care provided by others is indicated on a 5-point scale (completely dependent (1) - completely independent (5)); † Nosocomial pressure ulcers: Resident suffers from at least one nosocomial pressure ulcer category 2-4 ${ }^{12}$; Medication incidents: Resident had at least one medication incident during the last 30 days (Omitted dose, wrong dose, wrong time taken, wrong drug, wrong drug administration); Falls: Resident has fallen at least once during the last 30 days; Antipsychotic drug use: Antipsychotic drug use during last 7 days; Indwelling urinary catheter use: Resident has an indwelling urinary catheter in place at the time; $\neq$ significantly different among somatic and sychogeriatric wards ( $p<.01$; independent samples t-test or chi-square); $\S$ underlined score is the most favorable score 
Overall, a relationship between HPRD and clinical resident outcomes was not found. For each outcome, the multilevel and the fixed-effects logistic regression analyses were almost identical, and the ICC was low (ICC $\leq 0.04$ ). Therefore, only the results of the fixed-effects logistic regression are reported (Table 2). For residents from both ward types, the probability of experiencing nosocomial pressure ulcers, falls, antipsychotic drug use, or urinary indwelling catheters was not significantly associated with HPRD (with odds ratios between 0.80 and 1.94; Table 2). For residents living in psychogeriatric wards, however, higher HPRD were associated with a higher probability of experiencing a medication incident (odds ratio 3.93; 95\% Cl 1.27-12.18). This was not demonstrated for residents living in somatic wards (odds ratio 0.98). In addition, a relationship between HPRD and staff-reported QoC for both ward types was not found, as HPRD were not significantly associated with staff-reported QoC (with parameter estimates ranging from -0.22 to 0.09 ; Table 2 ). Due to the low \% of RNs in the participating wards, we were unable to estimate the relationship between staff mix and QoC.

Table 2: Associations between quantity of staff and quality of care indicators

\begin{tabular}{|c|c|c|c|c|}
\hline Clinical indicators $^{*}$ & Ward type & OR & $95 \% \mathrm{Cl}$ & p-value \\
\hline \multirow[t]{2}{*}{ Nosocomial pressure ulcers } & Somatic & 1.56 & $0.61-3.97$ & .36 \\
\hline & Psychogeriatric & 1.21 & $0.31-4.76$ & .79 \\
\hline \multirow[t]{2}{*}{ Medication incidents } & Somatic & 0.98 & $0.41-2.34$ & .97 \\
\hline & Psychogeriatric & 3.93 & $1.27-12.18$ & .02 \\
\hline \multirow[t]{2}{*}{ Falls } & Somatic & 1.94 & $0.84-4.50$ & .12 \\
\hline & Psychogeriatric & 1.59 & $0.81-3.10$ & .18 \\
\hline \multirow[t]{2}{*}{ Antipsychotic drug use } & Somatic & 1.23 & $0.59-2.55$ & .58 \\
\hline & Psychogeriatric & 0.98 & $0.61-1.57$ & .93 \\
\hline \multirow[t]{2}{*}{ Urinary indwelling catheter use } & Somatic & 0.90 & 0.51-1.59 & .72 \\
\hline & Psychogeriatric & 0.80 & $0.26-2.45$ & .70 \\
\hline Staff-reported indicators $^{\dagger}$ & Ward type & $b$ & SE & p-value \\
\hline \multirow[t]{2}{*}{ Grade overall quality of care } & Somatic (ICC: 0.41) & -0.22 & 0.33 & .52 \\
\hline & Psychogeriatric (ICC: 0.30) & 0.09 & 0.26 & .72 \\
\hline \multirow[t]{2}{*}{ Recommending the ward } & Somatic (ICC: 0.26) & -0.18 & 0.32 & .58 \\
\hline & Psychogeriatric (ICC: 0.34) & 0.04 & 0.32 & .91 \\
\hline
\end{tabular}

Note: $\mathrm{OR}=$ odds ratio; $95 \% \mathrm{Cl}=95 \%$ confidence interval around $\mathrm{OR}$; $\mathrm{SE}=$ standard error; ICC= intraclass correlation coefficient; * General logistic regression analyses: Fully adjusted models estimating the relationship between quantity of staff and clinical quality of care controlling for background characteristics, i.e. ward size, and residents' age, gender, length of stay, number of comorbidities, and care dependency status. + Multilevel linear regression analyses (random intercept): Fully adjusted models estimating the relationship between quantity of staff and staff-reported quality of care controlling for background characteristics, i.e. ward size and residents' mean age, gender ( $\%$ female), length of stay, number of comorbidities, and care dependency status 


\section{DISCUSSION}

Our data analyses showed that the quantity of nursing staff was not related to clinical indicators as well as staff-perceived QoC in nursing homes. This is in line with the conclusions drawn in prior European studies. ${ }^{7-10}$

Some methodological limitations should be considered. Due to the cross-sectional design, our findings should be interpreted carefully. Considering staff-reported QoC can be seen as a strength, as staffs' perception of QoC is an important addition to clinical outcomes. However, staff-reported QoC is based on the subjective views of individual staff members who may interpret the concept of QoC differently. Moreover, we were unable to test the relationship between staff mix and QoC. A strength of the study was that data were of good quality as we made use of an existing data infrastructure (LPZ), collected actual staffing data directly from ward rosters and all data were collected at the same point in time.

Our findings underscore that adding extra manpower will not per se lead to better QoC in European nursing homes. Nevertheless, probably there is a minimum HPRD threshold below which the probability of poor QoC outcomes is higher. However, focusing on quantity of nursing care alone might be too simple to improve QoC. The relationship between staffing and QoC seems to be more complex. Instead of focusing on the quantity of staff, one should therefore consider the quality (i.e., skills and competencies) of the team. In future studies, the quality of the team should be considered as well. Besides educational backgrounds of nursing staff, the ward environment and work processes might contribute to QoC in nursing homes. Tentative evidence suggests that better educated staff, a positive organizational culture and good teamwork, communication and coordination may lead to better QoC in nursing home wards. ${ }^{7}$

More information on the effects of ward environment (e.g., team climate) and work processes (e.g., communication or coordination), as well as the optimal allocation of nursing staff from different educational backgrounds is needed. 


\section{REFERENCES}

1. Giorgio LD, Filippini M, Masiero G. Is higher nursing home quality more costly? Eur J Health Econ. 2016;17:1011-1026

2. Backhaus R, Verbeek H, van Rossum E, Capezuti E, Hamers JPH. Nurse staffing impact on quality of care in nursing homes: a systematic review of longitudinal studies. J Am Med Dir Assoc. 2014;15:383-393.

3. Corazzini KN, Anderson RA, Mueller C, Thorpe JM, McConnell ES. Licensed practical nurse scope of practice and quality of nursing home care. Nurs Res. 2013;62:315-324.

4. Spilsbury K, Hewitt C, Stirk L, Bowman C. The relationship between nurse staffing and quality of care in nursing homes: A systematic review. Int J Nurs Stud. 2011;48:732-750.

5. Castle N. Nursing home caregiver staffing levels and quality of care. J App/ Gerontol. 2008;27:375-405.

6. Han K, Trinkoff AM, Storr CL, Lerner N, Johantgen M, Gartrell K. Associations between state regulations, training length, perceived quality and job satisfaction among certified nursing assistants: cross-sectional secondary data analysis. Int J Nurs Stud. 2014;51:1135-1141.

7. Zúñiga $F$, Ausserhofer D, Hamers JPH, Engberg S, Simon M, Schwendimann R. Are staffing, work environment, work stressors, and rationing of care related to care workers' perception of quality of care? A cross-sectional study. J Am Med Dir Assoc. 2015;16:860-866.

8. Huizing AR, Hamers JPH, De Jonge J, Candel M, Berger MP. Organisational determinants of the use of physical restraints: a multilevel approach. Soc Sci Med. 2007;65:924-933.

9. Havig AK, Skogstad A, Kjekshus LE, Romøren TI. Leadership, staffing and quality of care in nursing homes. BMC Health Serv Res. 2011;11:327.

10. Heeren P, Van de Water G, De Paepe L, Boonen S, Vleugels A, Milisen K. Staffing levels and the use of physical restraints in nursing homes: a multicenter study. J Gerontol Nurs. 2014;40:48-54.

11. Aiken LH, Sermeus W, Van den Heede K, et al. Patient safety, satisfaction, and quality of hospital care: cross sectional surveys of nurses and patients in 12 countries in Europe and the United States. BMJ. 2012;344:e1717.

12. Van Nie-Visser NC, Schols JMGA, Meesterberends E, Lohrmann C, Meijers JM, Halfens RJ. An international prevalence measurement of care problems: study protocol. J Adv Nurs. 2013;69:e18-e29.

13. Onder G, Carpenter I, Finne-Soveri H, et al. Assessment of nursing home residents in Europe: the Services and Health for Elderly in Long TERm care (SHELTER) study. BMC Health Serv Res. 2012;12:5.

14. Meijers JM, Candel MJ, Schols JM, Van Bokhorst-de van der Schueren MA, Halfens RJ. Decreasing trends in malnutrition prevalence rates explained by regular audits and feedback. J Nutr. 2009;139:1381-1386.

15. Meijers JM, Schols JM, Van Bokhorst-de van der Schueren MA, Dassen T, Janssen MA, Halfens RJ. Malnutrition prevalence in The Netherlands: results of the annual Dutch national prevalence measurement of care problems. Br J Nutr. 2009;101:417-423. 


\title{
CHAPTER 5
}

\author{
WORK ENVIRONMENT \\ CHARACTERISTICS ASSOCIATED \\ WITH QUALITY OF CARE IN DUTCH \\ NURSING HOMES: \\ A CROSS-SECTIONAL STUDY
}

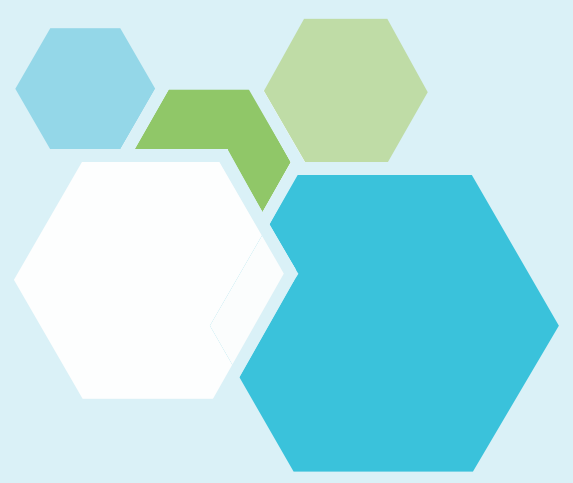

This chapter was published as:

Backhaus R, Van Rossum E, Verbeek H, Halfens RJG, Tan FES, Capezuti E, Hamers JPH.

Work Environment Characteristics Associated with Quality of Care in Dutch Nursing

Homes : A Cross-Sectional Study. International Journal of Nursing Studies 2017;66:15-22. 


\section{ABSTRACT}

Background: A lack of relationship between direct care staffing levels and quality of care, as found in prior studies, underscores the importance of considering the quality of the work environment instead of only considering staff ratios. Only a few studies, however, have combined direct care staffing with work environment characteristics when assessing the relationship with quality of care in nursing homes.

Objectives: To examine the relationship between direct care staffing levels, work environment characteristics and perceived quality of care in Dutch nursing homes.

Design: Cross-sectional, observational study in cooperation with the Dutch Prevalence Measurement of Care Problems

Settings: Twenty-four somatic and 31 psychogeriatric wards from 21 nursing homes in the Netherlands.

Participants: Forty-one ward managers and 274 staff members (registered nurses or certified nurse assistants) from the 55 participating wards.

Methods: Ward rosters were discussed with managers to obtain an insight into direct care staffing levels (i.e, total direct care staff hours per resident per day). Participating staff members completed a questionnaire on work environment characteristics (i.e., ward culture, team climate, communication and coordination, role model availability, and multidisciplinary collaboration) and they rated the quality of care in their ward.

Data were analyzed using multilevel linear regression analyses (random intercept). Separate analyses were conducted for somatic and psychogeriatric wards.

Results: In general, staff members were satisfied with the quality of care in their wards. Staff members from psychogeriatric wards scored higher on the statement 'In the event that a family member had to be admitted to a nursing home now, I would recommend this ward'. A better team climate was related to better perceived quality of care in both ward types ( $p \leq 0.020)$. In somatic wards, there was a positive association between multidisciplinary collaboration and agreement by staff of ward recommendation for a family member $(p=0.028)$. In psychogeriatric wards, a lower score on market culture ( $p$ $=0.019)$, better communication/coordination $(p=0.018)$ and a higher rating for multidisciplinary collaboration $(p=0.003)$ were significantly associated with a higher grade for overall quality of care. Total direct care staffing, adhocracy culture, hierarchy culture, as well as role model availability were not significantly related to quality of care. 
Conclusions: Our findings suggest that team climate may be an important factor to consider when trying to improve quality of care. Generating more evidence on which work environment characteristics actually lead to better quality of care is needed. 


\section{INTRODUCTION}

Nursing staff, including certified nurse assistants, nurse aides and registered nurses, provide most of the round-the-clock direct care in nursing homes. Even though the relationship between direct care staffing levels and quality of care (QoC) in nursing homes has been assessed in many, mostly US based, studies, the relationship is unclear as studies provide inconsistent conclusions. ${ }^{1-3}$ Worldwide, little progress has been made on establishing minimum nursing home staffing standards, whereas these might positively affect the $\mathrm{QoC}$ and quality of life of nursing home residents. ${ }^{4}$ However, improvements in the QoC in nursing homes in the future cannot simply focus on numbers and educational backgrounds of direct care staff. A lack of relationship between direct care staffing levels and QoC, as found in prior studies, underscores the importance of considering the quality of the work environment instead of just the staff ratios. ${ }^{1,5}$ Only a few studies, however, have combined direct care staffing with work environment characteristics when assessing the relationship with QoC in nursing homes. ${ }^{5,6}$ Therefore, comprehensive theoretical models, integrating direct care staffing and other work environment characteristics are scarce, ${ }^{7}$ while at the same time, evidence on the relationship between work environment characteristics and QoC increases. ${ }^{7}$

Different work environment characteristics have been identified as determinants for QoC in prior studies. For example, ward environment characteristics such as positive work culture and a good team climate have been associated with better QoC in nursing homes. ${ }^{7}$ Also, work processes like good communication and coordination among direct care staff have been associated with better QoC in nursing homes. ${ }^{8,9}$ Evidence from the hospital setting suggests that multidisciplinary collaboration, such as between nurses and physicians, might lead to better QoC as well, but evidence for the nursing home sector is still scarce. ${ }^{10}$ In the international literature, increasing attention is paid to the presence of role models as a determinant for QoC. A role model is a staff member whose work is emulated by other team members. ${ }^{11}$ In countries like the US and Canada, role modeling is considered part of advanced roles such as nurse practitioner, nurse consultant or nurse specialist. ${ }^{12}$ To our knowledge, the relationship between the presence of role models within a team and $\mathrm{QoC}$ in nursing homes has not been reported in the research literature. Nevertheless, we hypothesize that the presence of a role model in a ward might be associated with better QoC, as role models assist other direct care staff to deal more effectively with challenging or complex situations. ${ }^{13}$ Based on a literature review, we developed the model presented in Figure 1, suggesting that work environment characteristics might mediate the relationship between staffing levels and QoC. In this study, all these factors that possibly determine QoC in nursing homes, i.e., direct care staffing levels, ward environment characteristics (work culture, team climate), as well as work processes (communication and coordination, multidisciplinary collaboration, presence of role models) will be considered jointly when 
examining the relationship with QoC (Figure 1). In addition, specific attention will be paid to the selection of $\mathrm{QoC}$ outcomes. Nursing home QoC is predominantly operationalized as clinical outcomes for residents such as the prevalence of falls or medication incidents. Others have utilized staff perception of QoC, ${ }^{5}$ ince this has been found suitable in other settings. ${ }^{14}$ Using direct care staff members as informants of the overall QoC in nursing homes might be an underexplored opportunity, as they have insights into aspects of QoC that are not necessarily documented in medical records or resident files. ${ }^{15}$ Staff perception of QoC is not based on isolated components of QoC, e.g. a resident falls or there is a medication incident, but develops over time, and thus provides a more comprehensive view of the residents' care. ${ }^{5}$

The aim of our study is to examine the relationship between direct care staffing levels, work environment characteristics and perceived QoC in Dutch nursing homes. In our study, parts of the theoretical model presented in Figure 1 will be tested to infer this relationship.



Figure 1: Theoretical model of the relationship between direct care staffing levels, ward environment characteristics, work processes and staff-reported quality of care

Note: Bold arrows are tested in this study. 


\section{METHODS}

\section{Study design}

We conducted this study in cooperation with the Dutch Prevalence Measurement of Care Problems (LPZ: Landelijke Prevalentiemetingen Zorgproblemen). The LPZ database includes a cross-sectional point prevalence measurement of several care problems (such as pressure ulcers and medication incidents) and takes place annually on the same day in different healthcare settings. ${ }^{16}$ Health care organizations participate voluntarily. All nursing homes participating in the LPZ measurement in April 2014 were invited to participate in our study. Among nursing homes that agreed to participate, a cross-sectional, observational study was conducted between April and June 2014. The study was conducted in Dutch.

\section{Setting and participants}

Fifteen organizations with 44 nursing homes were invited to participate in our additional study. Within Dutch nursing homes, three different wards can be distinguished: somatic wards for residents with physical disabilities, psychogeriatric wards for residents with dementia, and rehabilitation wards that provide sub-acute rehabilitation. Somatic and psychogeriatric wards provide long-term, residential care, whereas rehabilitation wards provide short-term, skilled nursing care. In our study, we focused on somatic and psychogeriatric wards. Wards for residents with specific diseases such as Huntington's disease, were excluded. If the director of an invited organization gave consent to participate in the study, ward managers from this organization were invited to participate in a brief, voluntary interview concerning nurse staffing. Ward managers that gave informed consent were interviewed (via telephone). In addition, the ward managers were asked to distribute a digital questionnaire to at least five staff members working in their ward. The questionnaire was implemented using the online survey tool SurveyMonkey (www.surveymonkey.com) and included questions on ward environment characteristics, work processes, and staff-reported QoC. The choice to ask for at least five staff members and not for all staff members from a ward was made to keep participation in this study feasible. Staff members that were registered nurses or certified nurse assistants and worked at least 12 hours per week in one of the somatic or psychogeriatric wards were considered eligible for participation. The ward managers were asked to invite, to the extent possible, staff members with different educational background and years of work experience. All resident data were extracted from the LPZ database. Data about the residents were collected by two health care professionals, one who works on the resident's ward and one on another ward. These health care professionals received standardized training on how to collect resident level data. ${ }^{16}$ Good inter-rater reliability testing between the two observers has been previously reported (Cohen's kappa 0.87). ${ }^{17}$ 


\section{Data sources, variables and operationalization}

In Table 1, the main variables, their operationalization and the data sources are presented. All measurement instruments were selected based on their psychometric properties (validity, reliability), clinical utility and appropriateness for the Dutch nursing home setting and population. To the extent that it is possible, previously tested scales were used for measurement. The ward manager interview, the questionnaire completed by staff members and the information extracted from the LPZ database were in Dutch. Interview questions were pretested for comprehensibility with ward managers, and questions included in the questionnaire were pretested with direct care staff (registered nurses, certified nurse assistants) from two nursing home organizations that did not participate in our study.

\section{Resident characteristics}

Residents' age, gender, nursing home length of stay, number of comorbidities and care dependency status were extracted from the LPZ database (Table 1). Care dependency was assessed with the Care Dependency Scale (CDS). ${ }^{18}$ The CDS covers 15 activities for which the degree to which the resident is dependent upon care provided by others is indicated on a 5-point scale (completely dependent (1) - completely independent (5)): eating and drinking, incontinence, body posture, mobility, day/night pattern, getting dressed and undressed, body temperature, hygiene, avoiding danger, communication, contact with others, awareness of rules and values, daily activities, recreational activities, and learning ability. ${ }^{16}$ For each resident, the total score (sum of 15 items) was divided by 15 to obtain a mean score.

\section{Direct care staffing levels and ward size}

Data on direct care staffing levels and the ward size (number of residents living in ward) were collected via ward managers. Ward managers provided data both on how many residents were living in the ward and on the nurse staffing for an average day within the last 7 days. Based on the actual ward roster, this included the number of direct care staff members that worked on that day (morning (7:00 am - 12:00 noon), day (12:00 noon - 5:00 pm), evening (5:00 pm - 11:00 pm), and night (11:00 pm - 7:00 am) shifts), each staff member's educational background and the exact start and finish time of their shift. The educational background of direct care staff working in Dutch nursing home varies and is explained in Table 1. Data were obtained via (telephone) interviews and based on actual schedules. Total staff hours per resident day (HPRD) were calculated by dividing the total direct care staff hours for that specific day by the number of residents living in the ward. 
Table 1: Study variables and their measurement

\begin{tabular}{|c|c|}
\hline Variable & Measurement \\
\hline \multicolumn{2}{|l|}{ Ward characteristics } \\
\hline Total direct care staffing levels & $\begin{array}{l}\text { Total direct care staff hours per resident per day (HPRD): } \\
\text { Direct care staff consist of nurse aides, nurse assistants, certified } \\
\text { nurse assistants (comparable to licensed practical/vocational } \\
\text { nurses in the US }{ }^{19} \text { ), vocationally trained registered nurses, } \\
\text { baccalaureate-educated registered nurses, specially trained } \\
\text { feeding assistants, trainees, and untrained staff. }\end{array}$ \\
\hline Ward size & Number of residents living in ward \\
\hline Ward type & Psychogeriatric/somatic nursing care ward \\
\hline \multicolumn{2}{|l|}{ Resident characteristics } \\
\hline Gender & Man/woman \\
\hline Age & Age in years \\
\hline Length of stay & Number of days \\
\hline Comorbidities & $\begin{array}{l}\text { Number of comorbidities ( } \underline{0}-24 *) \text { : } \\
\text { Infectious illness; cancer; endocrine, nutritional or metabolic } \\
\text { illness/disease; diabetes mellitus; disease of blood or blood related organs; } \\
\text { psychological disorders; dementia; nervous system disorder (excluding } \\
\text { cerebrovascular accident (CVA)); spinal cord lesion/paraplegia; cardio } \\
\text { vascular disease; CVA/hemiparesis; respiratory disorder/diseases, including } \\
\text { nose and tonsils; disorder/disease of the digestive tract, including intestinal } \\
\text { obstruction, peritonitis, hernia, liver, gallbladder, pancreas; } \\
\text { disorder/disease of kidney/urinary tract, sexual organs; skin } \\
\text { disorder/disease; motor disorder/disease; congenital disorders; injury } \\
\text { resulting from accident(s), undesirable consequences of accident(s); } \\
\text { symptoms and abnormal clinical or lab findings, not elsewhere classified; } \\
\text { overdose/substance abuse/addiction; disease of the eye; disease of the } \\
\text { ear; pregnancy, child birth; external factors for disease }\end{array}$ \\
\hline Care dependency & Care Dependency Scale ${ }^{18}$ \\
\hline \multicolumn{2}{|l|}{ Ward environment characteristics } \\
\hline Organizational culture & $\begin{array}{l}\text { Four different culture types from the competing values } \\
\text { framework (CVF) for long-term care } \text { ch }^{20}\end{array}$ \\
\hline Team climate & Dutch 14-item version of the team climate inventory $(\mathrm{TCl})^{21}$ \\
\hline \multicolumn{2}{|l|}{ Work process characteristics } \\
\hline Communication/coordination & $\begin{array}{l}\text { Communication/coordination subscale from the Work } \\
\text { Environment and Perceived Work Effectiveness in Nursing Homes } \\
\text { questionnaire }\end{array}$ \\
\hline Role model availability & Employee has a role model working in the ward (yes/no) \\
\hline Multidisciplinary collaboration & Grade ranging from $1-\underline{10}^{*}$ \\
\hline \multicolumn{2}{|c|}{ Staff-reported quality of care outcomes } \\
\hline Grade overall quality of care & Grade ranging from $1-\underline{10}^{*}$ \\
\hline Recommending the ward & $\begin{array}{l}\text { "In the event that a family member had to be admitted to a } \\
\text { nursing home now, I would recommend this ward" (completely } \\
\text { not agree (1) - completely agree }(\underline{5}) \text { )* }\end{array}$ \\
\hline
\end{tabular}

Note: * underlined score is the most favourable score 


\section{Ward environment characteristics}

Ward environment characteristics (organizational culture, team climate) were assessed by a questionnaire completed by staff members (registered nurses or certified nurse assistants). Organizational culture was measured with the Dutch version ${ }^{20}$ of the competing values framework (CVF) for long-term care. ${ }^{22}$ The six CVF items cover six domains: dominant organizational characteristic, administration, management style, organizational 'glue' (i.e., relational characteristics that connect the ward members together, for example, 'loyalty, trust and commitment' or 'formal procedures, rules and policies'), strategic emphasis and criteria for success. For each domain, staff members had to rank order four statements from 1 to 4 , with 4 best describing the culture on their ward. Each statement belongs to a specific culture type: clan, adhocracy, market or hierarchy. ${ }^{20}$ Clan culture is associated with shared goals and values, strong cohesion and a sense of collective identity. Adhocracy culture is characterized by the ability to adapt quickly to new opportunities and deal successfully with changes. In a market culture, there is a focus on profit, competitiveness and productivity. A hierarchy culture is characterized by centralized decisions, structures and rules. ${ }^{20}$ Usually, more than one type of culture characterizes an organization. ${ }^{20,23}$ Therefore, we calculated a total score


Dutch applying back-translation and validated the scale in the Dutch nursing home setting. As the CVF is an ipsative or 'forced choice' scale and the scores on one subscale are dependent on scores on the other subscales, the internal consistency reliability cannot be tested. ${ }^{22}$ Team Climate was measured with the Dutch 14-item version of the Team Climate Inventory $(\mathrm{TCl}){ }^{21}$ The $\mathrm{TCl}$ measures four factors that contribute to the team climate: participative safety, support for innovation, vision, and task orientation. The underlying rationale is that effective team performance is often reached when team activities are characterized by these factors. ${ }^{24}$ Staff members had to score for each item on a 5-point scale (strongly disagree - strongly agree), with higher scores indicating a better team climate. The score for each item was summed up to determine a total scale score. Strating and Nieboer ${ }^{21}$ translated the TCl into Dutch and tested the construct validity, reliability, predictive validity and temporal stability of the scale, concluding that the psychometric properties were acceptable. For the four subscales of the $\mathrm{TCl}$, they found Cronbach's alphas ranging from 0.73-0.80. In our sample, the Cronbach's alpha for the total scale was 0.90 , indicating good internal consistency.

\section{Work processes}

In the questionnaire completed by staff members, work processes were assessed as well. Communication and coordination were measured with the communication/coordination subscale from the Work Environment and Perceived Work Effectiveness in Nursing Homes questionnaire that measures the degree to which communication between staff members is uninhibited, accurate, timely and effective, and focuses on effectiveness of procedures for coordinating tasks and job responsibilities'. ${ }^{9}$ This subscale consists of 15 items, which are scored on a 5-point scale (strongly disagree - strongly agree). The score for each item was summed up to determine a total scale score, with higher scale scores indicating better communication/coordination. No Dutch version of the Work Environment and 
Perceived Work Effectiveness in Nursing Homes questionnaire existed. To assure crosscultural validity, the communication and coordination subscale was translated according to the forward-backward translation guidelines from Beaton et al. ${ }^{25}$ The final Dutch version was tested for comprehensibility with direct care staff members (registered nurses, certified nurse assistants) to make sure that all items were appropriate for the Dutch nursing home setting. In our sample, the Cronbach's alpha of the scale was 0.80 , indicating acceptable internal consistency. To obtain insight into the existence of role models, staff members were asked whether or not they had a colleague (not necessarily a higher-educated colleague, but, for example, another certified nurse assistant) they viewed as a professional role model. In addition, the multidisciplinary collaboration between direct care staff, physicians and paramedics was graded by staff members (grade ranging from 1-10 with higher scores indicating better multidisciplinary collaboration).

\section{Staff-reported quality of care outcomes}

In the questionnaire completed by staff members, two questions on QoC were included. Participating staff members graded the overall QoC on their ward (grade 1-10, a higher score indicating better $\mathrm{QoC}$ ) and indicated to what extent they agreed with the statement "In the event that a family member had to be admitted to a nursing home now, I would recommend this ward" (completely not agree (1) - completely agree (5)).

\section{Statistical analyses}

Data analyses were performed with SPSS for Windows (version 22). Missing items were replaced by ward averages ( $n=2$ ) or respondents' mean score on the scales $(n=4)$. In addition, $8.9 \%(n=23)$ of the respondents did not rank order all statements within organizational culture domains correctly. Statements that were not rank ordered correctly were considered as missing and were imputed using multiple imputation techniques.

Differences in staff-reported QoC between somatic and psychogeriatric wards were found using independent samples t-tests, thus all subsequent analyses were considered separately. Means and standard deviations of ward, resident, staff, ward environment, work process characteristics as well as QoC were computed. To examine the relationship between direct care staffing levels, ward environment characteristics, work processes (independent variables) and staff-reported QoC (dependent variable), multilevel linear regression analyses (random intercept) were conducted, in which staff (level 1) was nested in wards (level 2). Intraclass correlation coefficients (ICC) were calculated to test the correlation between staff members working in the same ward. To test for multicollinearity among the independent variables, variance inflation factors (VIFs) were calculated. As all VIFs were lower than 5, no multicollinearity problem existed. ${ }^{26}$ For each dependent variable (staff-reported QoC outcomes), two different analyses were conducted. First, we conducted fully-adjusted analyses in which we controlled for background characteristics (i.e. ward size and residents' mean age, 
gender (female/male), length of stay, and number of comorbidities). Due to the low spread of the care dependency status variable, care dependency status was not considered as a background characteristic in the analyses. Second, we conducted unadjusted analyses in which we did not control for background characteristics. Likelihood ratios (-2LL) were considered to assess whether the adjusted or the unadjusted models fitted better (lower likelihood ratio values indicating a better fit).

\section{Ethical considerations}

The Medical Ethics Review Committee (METC) of the University Hospital Maastricht and Maastricht University approved the study protocol (METC14-4-057). Participation was voluntary and anonymous, and participants were informed that their answers would be treated as strictly confidential. No identifying information on the participants was collected. Ward managers had no access to the questionnaires completed by staff members and did not know which staff members had or had not completed the questionnaire. All data about the residents were extracted from an existing database (LPZ).

\section{RESULTS}

\section{Descriptive statistics}

Eight out of fifteen invited nursing home organizations participated in our study. The study was conducted in 21 nursing homes and 55 wards (31 psychogeriatric and 24 somatic). In total, 1438 residents were living in the 55 included wards, 617 lived in a somatic and 821 in a psychogeriatric ward. Ward and resident characteristics are described in Table 2.

\section{Staff characteristics}

Staff members amounting to 274 from the 55 included wards completed the questionnaire of ward environment and work process characteristics; 17 respondents were excluded from the analyses, as they did not complete the digital questionnaire. In total, 257 respondents were included in the analyses, of whom $84.0 \%$ were certified nurse assistants, $13.6 \%$ vocationally trained registered nurses, and $2.3 \%$ baccalaureateeducated registered nurses (Table 2).

\section{Ward environment and work process characteristics}

Staff members from somatic as well as psychogeriatric wards gave high mean ratings for the team climate (Table 2). For both types of wards, staff members scored, on average, highest on clan culture, followed by hierarchy, adhocracy and market culture. Also, the communication and coordination in their wards were rated as good. $73 \%$ of somatic ward staff members and $71 \%$ of psychogeriatric ward staff members indicated that they 
had a colleague whom they saw as role model for themselves. In general, staff members were satisfied with the collaboration among the various disciplines.

Table 2: Differences in study variable characteristics among somatic and psychogeriatric wards

\begin{tabular}{|c|c|c|}
\hline & $\begin{array}{l}\text { Somatic wards } \\
\qquad(n=24)\end{array}$ & $\begin{array}{l}\text { Psychogeriatric wards } \\
\qquad(n=31)\end{array}$ \\
\hline \multicolumn{3}{|l|}{ Ward characteristics $(n=55)$} \\
\hline Total direct care staffing (HPRD; mean, SD) & $3.06 \pm 0.58$ & $3.15 \pm 0.40$ \\
\hline Ward size (mean, SD) & $27 \pm 8$ & $27 \pm 10$ \\
\hline Resident characteristics & 617 residents & 821 residents \\
\hline Age in years (mean, SD) & $79 \pm 11$ & $83 \pm 9$ \\
\hline Female (\%) & 65 & 72 \\
\hline Length of stay in years and days (mean, SD) & $2.7(1,002 \pm 1,225)$ & $2.6(959 \pm 873)$ \\
\hline Number of comorbidities (mean, SD) & $3 \pm 1$ & $3 \pm 1$ \\
\hline Care dependency (scale range: $1-5 ;$ mean, SD) ${ }^{a}$ & $2.7 \pm 1.1$ & $2.1 \pm 1.1$ \\
\hline Staff characteristics & 104 staff members & 153 staff members \\
\hline Number of work hours per week (mean, SD) & $29.45 \pm 5.17$ & $29.18 \pm 5.48$ \\
\hline \multicolumn{3}{|l|}{ Educational background } \\
\hline Baccalaureate-educated RN (\%) & $2.9(n=3)$ & $2.0(n=3)$ \\
\hline Vocationally trained RN (\%) & $18.3(n=19)$ & $10.5(n=16)$ \\
\hline Certified nurse assistant (\%) & $78.8(n=82)$ & $87.6(n=134)$ \\
\hline \multicolumn{3}{|l|}{ Ward environment characteristics } \\
\hline Team climate (scale range: 14-70; mean, SD) & $53 \pm 7$ & $55 \pm 6$ \\
\hline Clan culture (scale range: 6-24; mean, SD) & $18 \pm 3(n=100)$ & $19 \pm 3(n=134)$ \\
\hline Adhocracy culture (scale range: 6-24; mean, SD) & $14 \pm 3(n=100)$ & $15 \pm 3(n=134)$ \\
\hline Market culture (scale range: 6-24; mean, SD) & $11 \pm 4(n=100)$ & $10 \pm 3(n=134)$ \\
\hline Hierarchy culture (scale range: 6-24; mean, SD) & $17 \pm 3(n=100)$ & $16 \pm 3(n=134)$ \\
\hline \multicolumn{3}{|l|}{ Work process characteristics } \\
\hline Communication/coordination (scale range: 15-75; mean, SD) & $54 \pm 7$ & $56 \pm 6$ \\
\hline Role model (\% staff having a role model) & 73 & 71 \\
\hline Multidisciplinary collaboration (scale range: 1-10; mean, SD) & $7.3 \pm 0.8$ & $7.2 \pm 0.9$ \\
\hline Staff-reported quality of care outcomes & 104 staff members & 153 staff members \\
\hline Grade overall quality of care (scale range: $1-10 ;$ mean, SD)* & $7.4 \pm 0.9$ & $7.7 \pm 0.7$ \\
\hline Recommending the ward (scale range: $1-5$; mean, SD)* & $3.5 \pm 1.0$ & $3.9 \pm 0.9$ \\
\hline
\end{tabular}

Note: $\mathrm{SD}=$ standard deviation $;{ }^{\text {a }}$ degree to which the resident is dependent upon care provided by others is indicated on a 5-point scale (completely dependent (1) - completely independent (5)); * significantly different among somatic and psychogeriatric wards $(p<.01$; independent samples t-test) 
Staff-reported quality of care

Overall, staff members from both somatic and psychogeriatric wards were satisfied with the overall QoC in their wards, while staff members working in psychogeriatric wards gave, on average, higher ratings (Table 2). More staff members from psychogeriatric wards agreed with the statement 'In the event that a family member had to be admitted to a nursing home now, I would recommend this ward', with a mean score of 3.9 from psychogeriatric staff compared to 3.5 from somatic staff (scale range: completely not agree (1) - completely agree (5)).

\section{Factors influencing quality of care in nursing homes}

The results of the multilevel regression analyses are reported in Table 3. As the - $2 \mathrm{LL}$ was lower for the unadjusted models and the adjusted models were not significantly better (corresponding p-values > 0.05), unadjusted models should be preferred. The parameter estimates from the adjusted (controlling for ward size, residents' mean age, gender (female/male), length of stay, and number of comorbidities) and unadjusted models were comparable. In both ward types, somatic and psychogeriatric, team climate was associated with QoC. Better team climate was significantly related to better staff perceptions for overall QoC ( $p=0.003$ and $p=0.020)$ and agreement by staff of ward recommendation for a family member $(p=0.000$ and $p=0.009)$. In somatic wards, there was a positive association between multidisciplinary collaboration and agreement by staff of ward recommendation for a family member $(p=0.028)$. In psychogeriatric wards, a lower score on market culture ( $p=0.019$ ), better communication/coordination $(p=0.018$ ) and a higher rating for multidisciplinary collaboration ( $p=0.003$ ) were significantly associated with a higher grade for overall QoC. Total direct care staffing, adhocracy culture, hierarchy culture, as well as role model availability were not significantly related to QoC. 


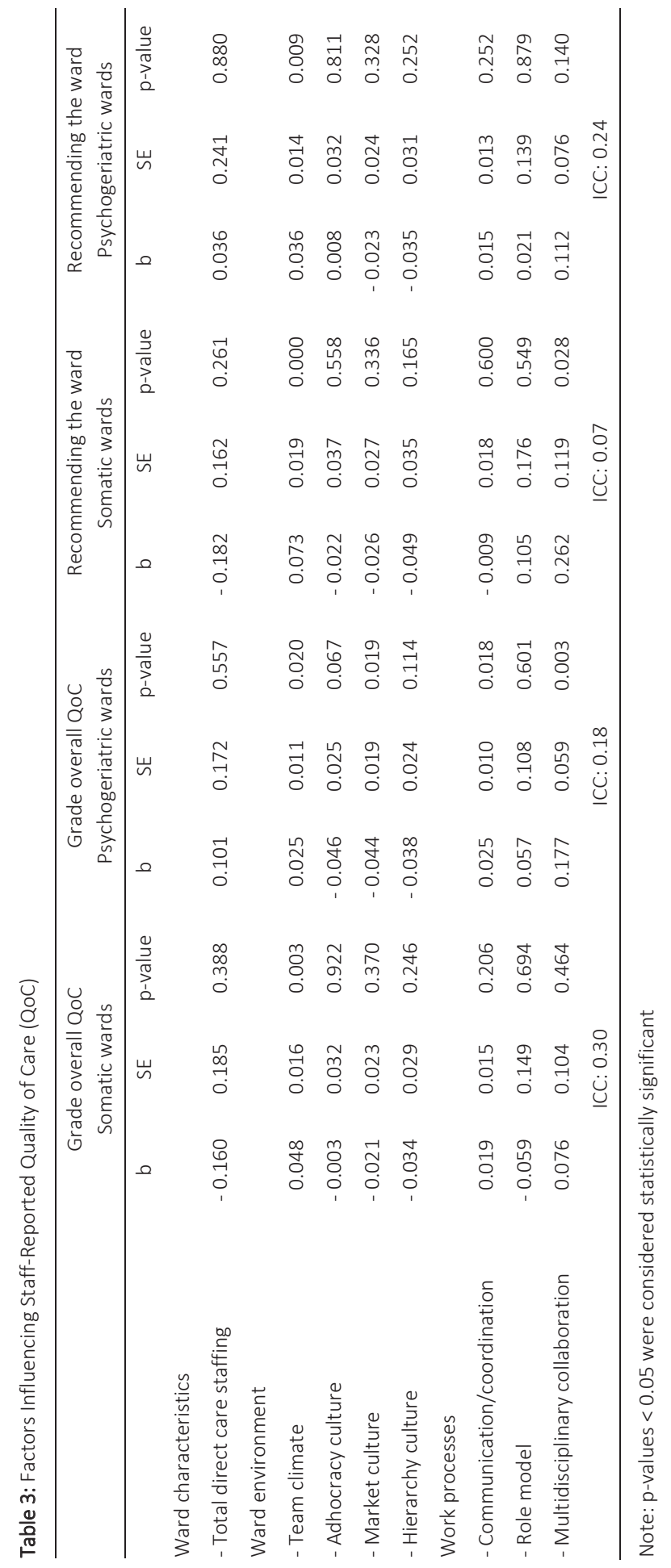




\section{DISCUSSION AND CONCLUSION}

Overall, the findings of this study only partly confirm our theoretical model (Figure 1). In our study, team climate was the only factor consistently associated with staff-reported QoC. Significant associations were also found for market culture, communication/coordination, and multidisciplinary collaboration, although these were not consistent across ward types. Contrary to our expectations, no significant associations were found for total direct care staffing, adhocracy culture, hierarchy culture and role model availability.

On average, staff members from both somatic and psychogeriatric wards were satisfied with the overall QoC in their wards. This is in agreement with the findings from van Beek and Gerritsen, ${ }^{20}$ who found that $72 \%$ of staff members rated the QoC in their ward as good to very good. Also, in a recent Swiss study, ${ }^{5} 93 \%$ of staff members perceived the QoC in their ward as good. Our finding that team climate was consistently associated with staff-reported QoC is similar to those of Zúñiga et al., ${ }^{5}$ who found that 'teamwork and safety climate' was the most important factor associated with staffreported QoC. Bosch et al. ${ }^{24}$ assessed the team climate in 67 Dutch nursing home wards in 2005. Even though they did not find a relationship between team climate and QoC, respondents' scores on team climate were comparable with the high mean ratings for team climate found in our study. Prior evidence suggests that a better team climate is not only associated with better QoC, but also with higher job satisfaction of direct care staff working in nursing homes. ${ }^{27}$

In psychogeriatric wards, a lower score on market culture was associated with a higher grade for overall QoC. In a study from van Beek and Gerritsen, ${ }^{20}$ conducted among Dutch psychogeriatric nursing home wards, market culture was also negatively associated with staff perceived QoC. As was the case with our study, staff members scored, on average, highest on clan culture, followed by hierarchy, adhocracy and market culture. In addition, better communication and coordination were significantly related to a higher rating for overall QoC in psychogeriatric wards. Compared with the findings from Temkin-Greener et al., ${ }^{9}$ staff members in our study perceived the communication and coordination in their wards as somewhat better. Good communication and coordination may improve QoC as it allows for timely responses to changes in residents' health, functional or mental status, as well as timely revisions in residents' care plans. ${ }^{28}$

In both ward types, a higher grade for multidisciplinary collaboration was associated with better perceived QoC. In somatic wards, better multidisciplinary collaboration was associated with higher scores on ward recommendation and in somatic wards with a higher grade for overall QoC. The fact that staff members in general were satisfied with the multidisciplinary collaboration might be partly explained by the employment pattern of physicians and other health professionals in Dutch nursing homes. In the 
Netherlands, medical nursing home care is provided by specifically trained nursing home medical specialists, who are, like all other health professionals (e.g., psychologists, physical therapists, speech therapists), employed by the nursing home. ${ }^{29}$ This might lead to a more coherent collaboration between professionals from different disciplines.

We were unable to demonstrate any relationship between total direct care staffing, adhocracy culture, hierarchy culture, or role model availability and staff-reported QoC. Also, in the study from Zúñiga et al., ${ }^{5}$ total direct care staffing levels were not associated with staff-reported QoC. This may indicate that staff satisfaction may not be improved by adding extra manpower. In a recent study conducted among Dutch hospital nurses, ${ }^{30}$ nurses reported that they were more dissatisfied with their role than with the actual staffing levels, as they felt a lack of authority and autonomy in decision-making. Prior studies indicated that having autonomy within the workplace enhanced the care provided by nurses. ${ }^{31,32}$ For example, in the study from McCabe et al., ${ }^{31}$ staff's autonomy was associated with self-efficacy and confidence in working with aged care residents. The fact that we were unable to demonstrate a relationship between adhocracy as well as hierarchy culture and staff-reported QoC confirms the findings from van Beek and Gerritsen. ${ }^{20}$ Also, role model availability was not significantly related to staff-reported QoC. One explanation for the lack of effect might be that we only distinguished between staff members that did or did not have a colleague they saw as role model for themselves, not considering, for example, the extent to which staff members are actually motivated and inspired by their role model. In addition, in many cases, no registered nurses were working in the wards. Registered nurses may be more suitable as role models compared to certified nurse assistants.

The findings of this study should be interpreted carefully. Due to the cross-sectional design we could only assess associations and not imply any cause and effect relationships. Moreover, as the largest proportion of staff members consisted of certified nurse assistants and only a few registered nurses were working in the participating wards, we were unable to assess the relationship between the staff mix (i.e., percentage of registered nurses working in a ward) and staff-reported QoC. In some wards, no registered nurses completed the questionnaire, meaning that only the perspective of certified nurse assistants could be considered. Even though we provided ward managers with criteria for the selection of participating staff members (i.e., different educational background and years of experience), we cannot ensure that the staff members chosen by the ward managers were representative of all staff members that worked in a ward. Regarding staff-reported QoC as outcome variables of our study, a potential weakness may be that staff members interpret the concept of QoC differently based on their individual perceptions. In addition, especially the certified nurse assistants may be unable to recognize all QoC deficits in their wards. Due to the fact that only a few registered nurses participated, we were unable to test for differences in QoC reported by registered nurses and certified nurse assistants. 
However, Zúñiga et al. ${ }^{5}$ did not find differences in the ratings of registered nurses and nurse aides. A strength of the study was that we collected actual staffing data directly from the ward managers and all data were collected at the same point in time.

Our proposed theoretical model should further be refined in future longitudinal studies, considering work environment characteristics as potential mediators. Future studies could consider a combination of staff-reported QoC and clinical resident outcomes. Generating more evidence on which work environment characteristics actually lead to better QoC in nursing homes may help to improve QoC in future nursing homes, as this knowledge would enable ward managers to select better targeted improvement strategies. Our findings suggest that team climate may be an important factor ward managers should consider when trying to improve QoC in their wards. 


\section{REFERENCES}

1. Backhaus R, Verbeek H, Van Rossum E, Capezuti E, Hamers JPH. Nurse staffing impact on quality of care in nursing homes: a systematic review of longitudinal studies. J Am Med Dir Assoc. 2014;15;383-393.

2. Bostick JE, Rantz MJ, Flesner MK, Riggs CJ. Systematic review of studies of staffing and quality in nursing homes. J Am Med Dir Assoc. 2006; 7:366-376.

3. Spilsbury K, Hewitt C, Stirk L, Bowman C. The relationship between nurse staffing and quality of care in nursing homes: a systematic review. Int J Nurs Stud. 2011;48:732-750.

4. Mueller C, Arling G, Kane R, Bershadsky J, Holland D, Joy A. Nursing home staffing standards: their relationship to nurse staffing levels. Gerontologist. 2006;46:74-80.

5. Zúñiga F, Ausserhofer D, Hamers JPH, Engberg S, Simon M, Schwendimann R. Are staffing, work environment, work stressors, and rationing of care related to care workers' perception of quality of care? A cross-sectional study. J Am Med Dir Assoc. 2015;16:860-866

6. Flynn L, Liang Y, Dickson GL, Aiken LH. Effects of nursing practice environments on quality outcomes in nursing homes. J Am Geriatr Soc. 2010;58:2401-2406.

7. Schwendimann R, Zúñiga F, Ausserhofer D, Schubert M, Engberg S, De Geest S. Swiss Nursing Homes Human Resources Project (SHURP): protocol of an observational study. J Adv Nurs. 2014;70:915-926.

8. Colon-Emeric CS, McConnell E, Pinheiro SO, et al. CONNECT for better fall prevention in nursing homes: results from a pilot intervention study. J Am Geriatr Soc. 2013;61:2150-2159.

9. Temkin-Greener $\mathrm{H}$, Zheng N, Katz P, Zhao H, Mukamel DB. Measuring work environment and performance in nursing homes. Med Care. 2009;47:482-491.

10. Van Bogaert $P$, Timmermans $O$, Weeks SM, Van Heusden D, Wouters K, Franck E. Nursing unit teams matter: Impact of unit-level nurse practice environment, nurse work characteristics, and burnout on nurse reported job outcomes, and quality of care, and patient adverse events - a cross-sectional survey. Int J Nurs Stud. 2014;51:1123-1134.

11. Johnson J. Nursing professional development specialists as role models. J Nurses Prof Dep. 2015;31:297299.

12. Elliott N, Begley C, Sheaf G, Higgins A. Barriers and enablers to advanced practitioners' ability to enact their leadership role: A scoping review. Int J Nurs Stud. 2016;60:24-45.

13. Backhaus R, Verbeek H, Van Rossum E, Capezuti E, Hamers JPH. Future distinguishing competencies of baccalaureate-educated registered nurses in nursing homes. Geriatr Nurs. 2015;36:438-444.

14. Aiken LH, Sermeus W, Van den Heede K, et al. Patient safety, satisfaction, and quality of hospital care: cross sectional surveys of nurses and patients in 12 countries in Europe and the United States. BMJ. 2012;344:e1717.

15. McHugh MD, Stimpfel AW. Nurse reported quality of care: a measure of hospital quality. Res Nurs Health. 2012;35:566-575.

16. Van Nie-Visser NC, Schols JMGA, Meesterberends E, Lohrmann C, Meijers JMM, Halfens RJG. An International prevalence measurement of care problems: study protocol. J Adv Nurs. 2013;69:e18-e29.

17. Meijers JM, Schols JM, Van Bokhorst - de van der Schueren MA, Dassen T, Janssen MA, Halfens RJ. Malnutrition prevalence in the Netherlands: results of the annual Dutch national prevalence measurement of care problems. Br J Nutr. 2009;101:417-423.

18. Dijkstra A, Yont GH, Korhan EA, Muszalik M, Kedziora-Kornatowska K, Suzuki M. The care dependency scale for measuring basic human needs: an international comparison. J Adv Nurs. 2012;68:2341-2348.

19. Verkaik R, Francke AL, Van Meijel B, Spreeuwenberg PMM, Ribbe MW, Bensing JM. The introduction of a nursing guideline on depression at psychogeriatric nursing home wards: effects on certified nurse assistants. Int J Nurs Stud. 2011;48:710-719.

20. Van Beek AP, Gerritsen DL. The relationship between organizational culture of nursing staff and quality of care for residents with dementia: questionnaire surveys and systematic observations in nursing homes. Int J Nurs Stud. 2010;47:1274-1282. 
21. Strating MM, Nieboer AP. Psychometric test of the Team Climate Inventory-short version investigated in Dutch quality improvement teams. BMC Health Serv Res. 2009;9:126.

22. Scott-Cawiezell J, Jones K, Moore L, Vojir C. Nursing home culture: a critical component in sustained improvement. J Nurs Care Qual. 2005;20:341-348.

23. Scott T, Mannion R, Marshall M, Davies $H$. Does organisational culture influence health care performance? A review of the evidence. J Health Serv Res Policy. 2003;8:105-117.

24. Bosch M, Halfens RJG, Van der Weijden T, Wensing M, Akkermans R, Grol R. Organizational culture, team climate, and quality management in an important patient safety issue: nosocomial pressure ulcers. Worldviews Evid Based Nurs. 2011;8:4-14.

25. Beaton DE, Bombardier C, Guillemin F, Ferraz MB. Guidelines for the process of cross-cultural adaptation of self-report measures. Spine. 2000;25:3186-3191.

26. García CB, García J, López Martín MM, Salmerón R. Collinearity: revisiting the variance inflation factor in ridge regression. J App/ Stat. 2015;42:648-661.

27. Schwendimann R, Dhaini S, Ausserhofer D, Engberg S, Zúñiga F. Factors associated with high job satisfaction among care workers in Swiss nursing homes - a cross sectional survey study. BMC Nurs. 2016;15:1-10.

28. Zheng NT, Temkin-Greener H. End-of-life care in nursing homes: the importance of CNA staff communication. J Am Med Dir Assoc. 2010;11:494-499.

29. Huls M, De Rooij SE, Diepstraten A, Koopmans R, Helmich E. Learning to care for older patients: hospitals and nursing homes as learning environments. Med. Educ. 2015;49:332-339.

30. Van Oostveen CJ, Mathijssen E, Vermeulen H. Nurse staffing issues are just the tip of the iceberg: a qualitative study about nurses' perceptions of nurse staffing. Int J Nurs Stud. 2015;52:1300-1309.

31. McCabe MP, Mellor D, Karantzas G, Von Treuer K, Davison TE, O'Connor D. Organizational factors related to the confidence of workers in working with residents with dementia or depression in aged care facilities. Aging Ment Health. 2015;1-7.

32. Suhonen R, Charalambous A, Stolt M, Katajisto J, Puro M. Caregivers' work satisfaction and individualised care in care settings for older people. J Clin Nurs. 2013;22:479-490. 



\section{CHAPTER 6}

\section{FUTURE DISTINGUISHING \\ COMPETENCIES OF \\ BACCALAUREATE-EDUCATED \\ REGISTERED NURSES IN \\ NURSING HOMES}

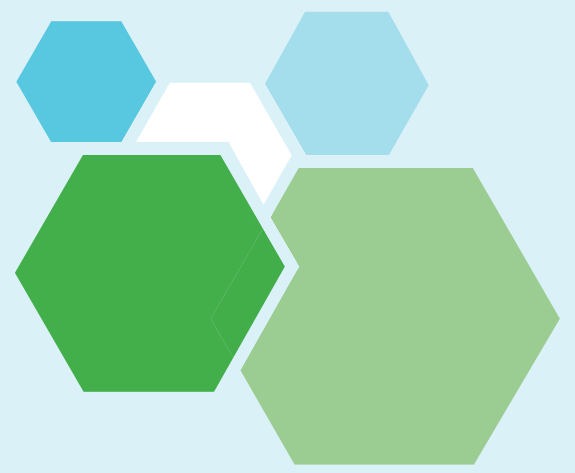

This chapter was published as:

Backhaus R, Verbeek H, Van Rossum E, Capezuti E, Hamers JPH. Future Distinguishing Competencies of Baccalaureate-Educated Registered Nurses in Nursing Homes. 


\section{ABSTRACT}

In view of the likelihood that the complexity of care required by those admitted to nursing homes will continue to increase, an expert consensus study was conducted to reach consensus on the competencies which distinguish baccalaureate-educated registered nurses from other nursing staff working in nursing homes. Thirty-one international experts, identified through literature and our professional network, participated in a two-round web-based survey and an expert meeting. Experts reached consensus on 16 desirable competencies, including some not traditionally associated with nursing expertise e.g. being a team leader, role model and coach within the nursing team. These findings suggest that revision of current nursing curricula, nurse training programs and nursing home job profiles might be needed to meet the medically and psychologically complex needs of nursing home residents. 


\section{INTRODUCTION}

Worldwide, the aging of the population will increase demand for long-term care services. ${ }^{1,2}$ Older adults want to stay at their own homes as long as possible, so only the most frail and dependent residents enter nursing homes. ${ }^{3,4}$ In the future nursing homes will not only have to grapple with higher demand for services and the increasingly complex needs of residents, but also with significant workforce shortages and poorly trained staff. ${ }^{2}$ The role of nursing staff is likely to become more complex as there is a trend to offer residents more personal choice, the diversity of care options is increasing and technological innovations continue. ${ }^{5}$ Future nursing home employees are likely to work with robotic care providers making continuous use of new electronic monitoring and decision-making systems. ${ }^{5}$ In addition to the shift to home and community based care for long-term living and support, other significant changes are occurring in health care systems worldwide that are influencing the resident population in nursing homes. ${ }^{5,6}$ First, an increase in the use of sub-acute care for short term rehabilitation (v. medical-surgical or rehabilitation hospital) is resulting in more residents with acute needs and an increase in the flow of residents in and out of the nursing home. ${ }^{7}$ Second, long-stay residents are most likely to have moderate to severe dementia. ${ }^{8}$ Other changes are country-specific. For example in the United States (US), there is a growth in accountable care organizations (ACOs) requiring nursing homes to be preferred partners in health systems as well as an increased focus on nursing home quality benchmarks., In the Netherlands, nursing home care is increasingly organized in smallscale and homelike environments, especially for people with dementia, in which nursing staff have integrated tasks. ${ }^{10}$

These changes demand explicit competencies of highly-skilled staff. Recent evidence suggests that an increase in baccalaureate-educated registered nurses (BRNs) leads to better quality of care $(\mathrm{Q} O \mathrm{C})$ in hospitals, ${ }^{11}$ but the impact on nursing home care is less clear. $^{12,13}$ We opine that an increase in the proportion of BRNs would lead to an improvement in quality of life and QoC for nursing home residents. We assume that BRNs in nursing homes, similar to BRNs in hospitals, may be better able to deal with the more complex care needs and could supervise less educated staff. However budget constraints have meant that there are few registered nurses (RNs) working in nursing homes and little is known about their unique contribution to QoC. ${ }^{14}$

As in many countries few RNs hold a baccalaureate degree, BRNs are an especially scarce resource in nursing homes, so obtaining more information on how to allocate them in nursing homes is desirable. Understanding how best to allocate BRNs depends on reaching a consensus on the competencies, i.e. skills, knowledge and attitudes, which will, in future, distinguish BRNs from other nursing staff. An understanding of what distinguishes BRNs from other nursing staff would help nursing home administrators to make informed decisions about human resource allocation ${ }^{15}$ and could also inform future BRN educational programs. When reviewing current 
competency profiles, we found that information was lacking on specific competencies for BRN in nursing homes, although worldwide, a tremendous amount of BRN competency profiles exists. For example in the US, there are more than 600 registered nurse to bachelor of science in nursing (RN-BSN) programs alone. ${ }^{16}$ However, BRN competency profiles in the US are often based on a general profile, the American Association of Colleges of Nursing (AACN) Essentials, ${ }^{17}$ not mentioning setting-specific (i.e. nursing home) competencies for BRNs. We identified only four existing BRN-specific competency profiles ${ }^{18-21}$ that are focusing on the care for older adults (Table 1). Although different nurse competency profiles for dementia care exist, none specifically addresses BRNs. ${ }^{22}$ Only few profiles exist that focus on the nursing home setting, for example the "Nursing home culture change competencies for nurses". 23 Distinguishing profiles for BRNs are missing.

Table 1: Overview of limitations of existing BRN competency profiles for the care of older adults

\begin{tabular}{|c|c|c|c|c|c|c|}
\hline $\begin{array}{l}\text { Name of the } \\
\text { BRN } \\
\text { competency } \\
\text { profile }\end{array}$ & Country/Year & Developed by & $\begin{array}{l}\text { Specific for } \\
\text { nursing home } \\
\text { setting }\end{array}$ & $\begin{array}{l}\text { Distinguishing } \\
\text { competencies } \\
\text { of BRNs form } \\
\text { other nursing } \\
\text { staff }\end{array}$ & $\begin{array}{l}\text { Future- } \\
\text { relevancy } \\
\text { explicitly } \\
\text { mentioned }^{\text {a }}\end{array}$ & $\begin{array}{l}\text { Broad } \\
\text { overview of } \\
\text { competencies }^{b}\end{array}$ \\
\hline $\begin{array}{l}\text { Recommended } \\
\text { Baccalaureate } \\
\text { Competencies } \\
\text { and Curricular } \\
\text { Guidelines for } \\
\text { the Nursing } \\
\text { Care of Older } \\
\text { Adults }^{18}\end{array}$ & $\begin{array}{l}\text { United } \\
\text { States/ } \\
2010\end{array}$ & $\begin{array}{l}\text { American } \\
\text { Association of } \\
\text { Colleges of } \\
\text { Nursing / } \\
\text { Hartford Institute } \\
\text { for Geriatric } \\
\text { Nursing }\end{array}$ & 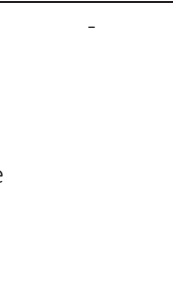 & - & $\checkmark$ & $\checkmark$ \\
\hline $\begin{array}{l}\text { Competencies } \\
\text { for the RN to } \\
\text { deliver person- } \\
\text { centered long- } \\
\text { term care }{ }^{19}\end{array}$ & $\begin{array}{l}\text { Canda/ } \\
2012\end{array}$ & $\begin{array}{l}\text { Research group } \\
\text { (McGilton et al.) }\end{array}$ & - & - & $\checkmark$ & - \\
\hline $\begin{array}{l}\text { Baccalaureate } \\
\text { Gerontological } \\
\text { and Geriatric } \\
\text { Nursing } \\
\text { Competence } \\
\text { Profile }^{20}\end{array}$ & $\begin{array}{l}\text { The Netherlands/ } \\
2012\end{array}$ & $\begin{array}{l}\text { Working group } \\
\text { (members from } \\
\text { educational and } \\
\text { health care } \\
\text { organizations) }\end{array}$ & - & - & $\checkmark$ & $\checkmark$ \\
\hline $\begin{array}{l}\text { Working areas } \\
\text { of } \\
\text { academically } \\
\text { qualified } \\
\text { registered } \\
\text { nurses }^{21}\end{array}$ & $\begin{array}{l}\text { Germany/ } \\
2014\end{array}$ & $\begin{array}{l}\text { German Nursing } \\
\text { Council/ German } \\
\text { Society of } \\
\text { Nursing Science }\end{array}$ & $\checkmark$ & $\checkmark$ & - & - \\
\hline
\end{tabular}

Note: ${ }^{a}$ Considering changes that are occurring in health care systems; ${ }^{b}$ i.e., not restricted to a specific area of expertise. 
The aim of this study was to obtain insight into the competencies, which should in the future, distinguish BRNs from other nursing staff (e.g. registered nurses or licensed nurses) in nursing homes. As nursing home systems develop ${ }^{5,24}$ it is important to ensure that BRNs are equipped with competencies that will enable them to meet future demands.

\section{METHOD}

We conducted an expert consensus study to obtain insight into the competencies which should, in the future, distinguish BRNs from other nursing staff. The study consisted of three different steps. First, a survey on future tasks and distinguishing competencies of BRNs in nursing homes was completed by expert panelists. Second, an expert meeting was held to discuss and interpret the findings of this survey with the expert panelists. Third, a final survey was completed by the expert panelists, aimed to determine the degree of consensus on the future distinguishing competencies of BRNs working in nursing homes.

\section{The expert panel}

Forty-one experts from various countries were identified through a literature search and our professional network. We defined an expert as an academically qualified person experienced in institutional long-term care and having expertise in the current work of BRNs in nursing homes in his or her country. We did not include BRNs since they would likely be influenced by their current working conditions and thus would have trouble envisioning alternative views of staff allocation. All identified experts were invited to participate in the consensus study.

\section{Survey on future tasks and distinguishing competencies of BRNs in nursing homes}

The survey was developed by our research team, who are all researchers in geriatrics. The survey had five parts. Part 1 asked about background information (age, gender, etc.). Part 2 consisted of questions on the current pattern of employment of BRNs in nursing homes (proportion of nursing home staff who are BRNs, current responsibilities of BRNs in the nursing home context). Part 3 was designed to elicit the respondent's opinions about the future of nursing homes, including opinions about the minimum and ideal proportion of nursing home staff that should be bachelor-educated in 2030 . In Part 4 the respondent described how he or she foresaw the responsibilities of BRNs in nursing homes in 2030, by answering questions such as "In your opinion, how much time will bachelor-educated registered nurses working in nursing home wards/units (in 
your country in the year 2030) in general spend on clinical responsibilities?". Part 5 asked the respondent to list 3-5 competencies specific to BRNs which he or she believed would be important in the nursing home context in 2030. Answering the questions in Parts 2 through 4 was intended to prompt the respondent to consider the competencies that should in future distinguish BRNs from other nursing staff.

All participating experts received an e-mail with a link to the digital survey which was implemented using the online survey tool Surveymonkey (http://www.surveymonkey.com). One researcher (RB) made a list of all competencies mentioned by the experts. The research team evaluated this initial list and deleted repetitions and combined similar competencies to eliminate redundancy. Next, the research team discussed all competencies and clustered them, based on their content, into 4 themes: leadership and coaching; communication; evidence-based practice; client assessment and geriatric expertise.

\section{Expert meeting to review the future distinguishing competencies mentioned by experts}

The experts who had completed the survey were invited to participate in an expert meeting, to discuss the analysis and interpretation of the survey results, paying particular attention to the competencies which would, in the future, distinguish BRNs from other nursing staff in nursing homes. The expert meeting was held during the November 2014 annual scientific meeting of the Gerontological Society of America. The two-hour discussion was led by a moderator (EvR) and two co-moderators (EC; JPHH). The discussion was audiotaped (and subsequently transcribed) while two other members of the research team (RB; HV) took contemporaneous notes.

Our analysis and the clustering into the 4 themes were discussed during the expert meeting. In addition, we asked which of the competencies on the list generated from the survey would really distinguish BRNs from other nursing staff with respect to each individual theme. Thinking about the specific contribution of BRNs to the care provided in nursing homes in the future helped the experts to answer these questions.

Final survey to determine the degree of consensus on the future distinguishing competencies

The list of distinguishing competencies, discussed and revised during the expert meeting, was presented in the final survey. Experts who completed the first survey received an e-mail with a link to the digital survey which was, again, implemented using the online survey tool Surveymonkey (http://www.surveymonkey.com). The experts were asked to assess each competency for application as a future role specific to BRNs in nursing homes. In line with other research we considered that consensus had been 
reached on a competency when there was at least $75 \%{ }^{25,26}$ agreement among the experts.

Based on a discussion during the expert meeting we also included five short questions about the (future) employment of master-educated registered nurses (advanced nursing roles such as nurse practitioners) in nursing homes in the survey.

\section{Ethical considerations}

The Medical Ethics Review Committee (METC) of the Atrium Medical Centre (METC Atrium-Orbis-Zuyd) approved the study protocol (METC number 14-N-97).

Participation in the study was voluntary. Participants were informed that their answers would be treated as strictly confidential and no identifying information about participants was collected. Participants in the expert meeting provided written informed consent to participation.

\section{RESULTS}

Thirty-six experts agreed to participate in the survey study; 31 completed the initial survey (86\%) and 28 (78\%) completed both surveys. Five experts participated in the expert meeting.

Initial survey on future tasks and distinguishing competencies of BRNs in nursing homes

Twenty-four of the 31 experts who responded to the initial survey were women. The mean age of survey respondents was 51 years (range: 39-66 years) and 27 were registered nurses. The experts came from four different continents (North America, Europe, Asia and Australia) and 14 different countries: United States (6), Canada (2), Germany (4), the Netherlands (4), Belgium (2), Sweden (2), Norway (1), Finland (1), Switzerland (1), United Kingdom (1), Spain (2), Australia (2), South Korea (2) and Taiwan (1).

In most countries only a small proportion of nursing staff in nursing homes were BRNs. Across our sample as a whole the average proportion of nursing home nursing staff with a bachelor degree was 17\% (range across countries: 1-70\%). On average, experts thought that by 2030 at least 39\% (range: 10-100\%) of the nursing staff in nursing homes should be bachelor-educated, and suggested that ideally this proportion should be 53\% (range: 15-100\%). Experts thought that in the future BRNs would spend a similar proportion of their time on clinical responsibilities (e.g. skilled nursing procedures) but less time on managerial responsibilities (e.g. supervision, staffing the 
ward/unit) and more time on specialist responsibilities (e.g. staff education, care innovation, quality management).

The experts identified 118 competencies distinguishing BRNs from other nursing staff. After eliminating redundancies this was reduced to a list of 38 competencies (see Table 2). Eighteen competencies were related to leadership and coaching, 3 to communication, 7 to evidence-based practice and 10 to client assessment and geriatric expertise. Frequently mentioned competencies included "being a leader on the ward/nursing unit," "being a staff and family coach/mentor," or "able to evaluate the situation of a client in its entirety." Our experts stressed that references to 'leadership' were not related to formal leadership positions, but to informal leadership as a role model and coach. 


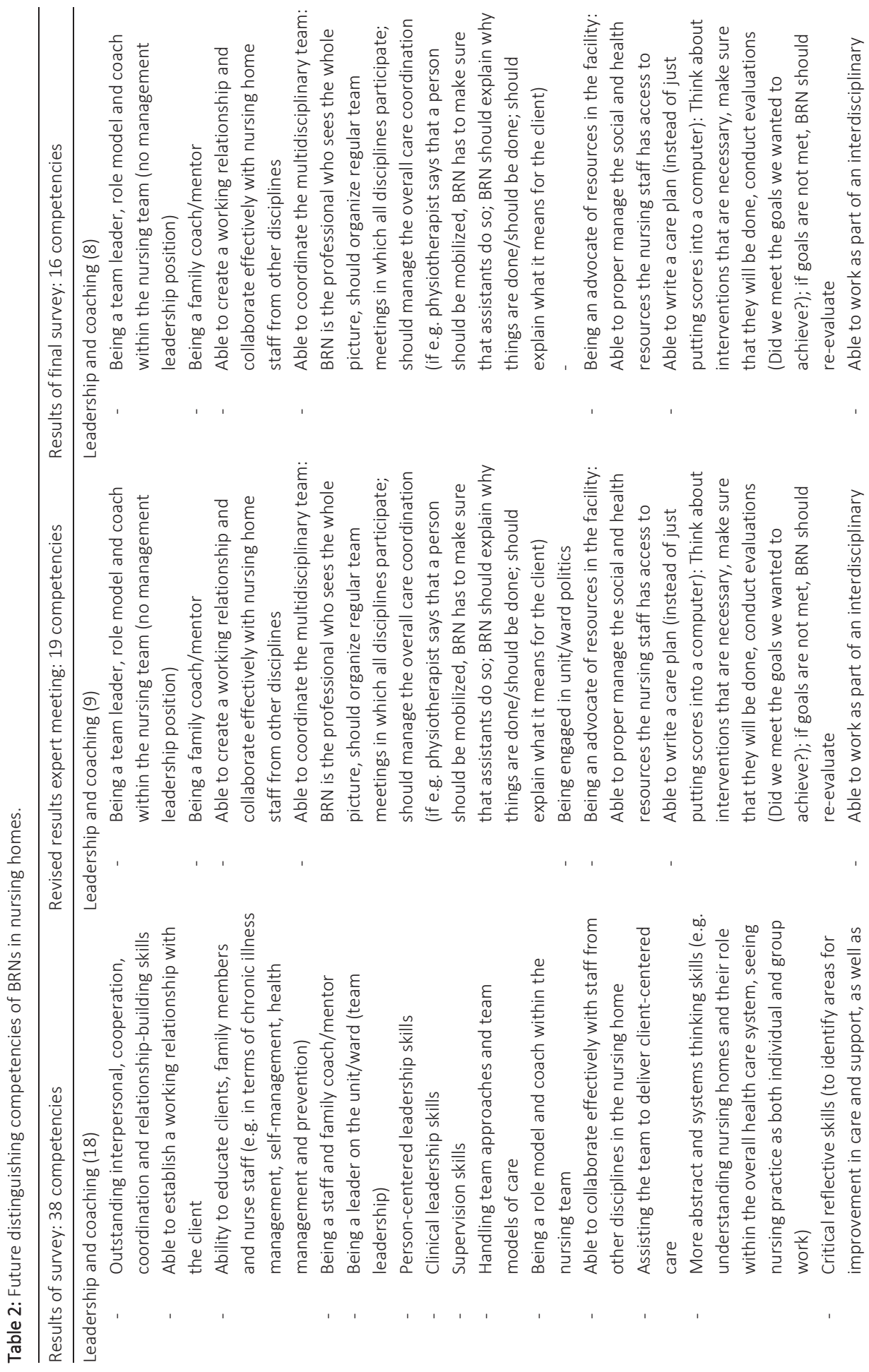




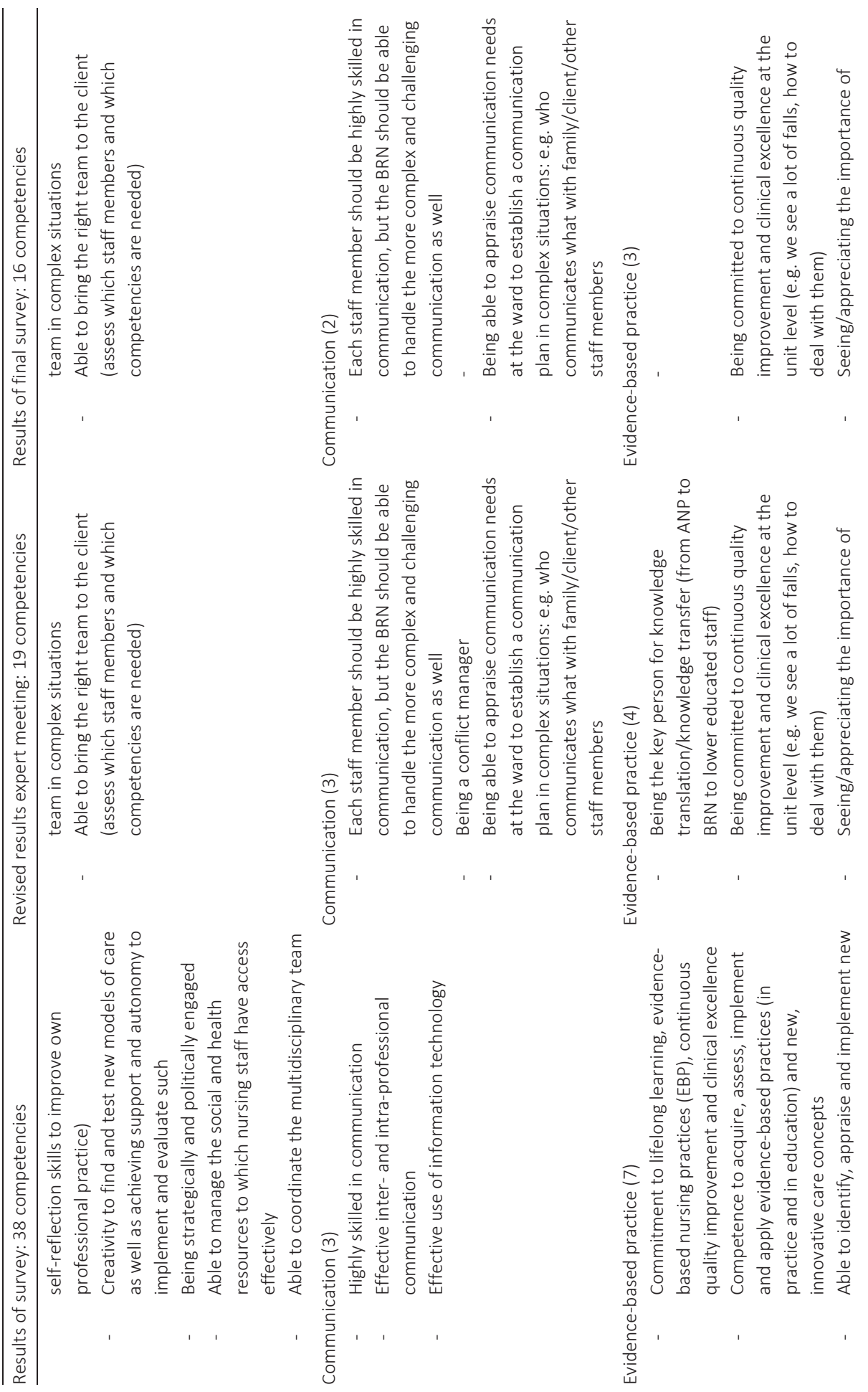














\section{Expert meeting to review the future distinguishing competencies mentioned by experts}

The participants of the expert meeting came from four different countries: United States (2), Canada (1), the Netherlands (1), and Switzerland (1).

During the expert meeting the clustering into the 4 themes was presented and discussed. From the list of 38 competencies, some competencies were discussed as being too general to be considered as distinguishing competency. Examples are "being strategically and politically engaged," "effective use of information technology," and "international exchange of ideas" (see Table 2). In addition, the experts suggested to revise the definitions of some competencies (see Table 2): for example, the competency "highly skilled in communication" was revised to "All staff should be highly skilled in communication, but the BRN should be able to handle the more complex and challenging communication as well." Based on the feedback from the experts, the research team reduced the list to 19 competencies.

The experts stressed that the competencies that distinguish BRNs from other nursing staff would depend partly on the context. Some competencies would only be relevant to specific settings (e.g. small-scale units, sub-acute rehabilitation etc.). The experts stressed that successful implementation of innovations in care practices would depend on collaboration between advanced practice nurses (APNs) and BRNs. Although nurses in both roles would be involved in front-line delivery of care, the experts saw the APNs as the clinical leaders, providing evidence on best practice and ensuring evidence informed delivery of care. APNs were seen as having more abstract and systems thinking skills, whereas it was envisioned that BRNs would oversee implementation of best practice guidelines at an operational level.

Final survey to determine the degree of consensus on the future distinguishing competencies

In the final survey, consensus was reached on 16 distinguishing competencies which would be desirable in BRNs working in nursing homes in the future. As Table 2 shows, half of the competencies (8) were related to leadership and coaching e.g. "being a team leader, role model and coach within the nursing team" or "able to create a working relationship and collaborate effectively with nursing home staff from other disciplines". Two of the remaining competencies were related to communication, three to evidencebased practice and three to client assessment and geriatric expertise.

The experts noted that the definitions of some competencies lacked clarity, as they were too general or were similar to other competencies. One expert suggested that examples of each competency should be provided. It was also emphasized that the BRN should be seen as part of a larger team: "Each member of the team has a distinct scope 
of practice and contribution to make, but in the end, all of them are responsible for the care of the resident and the family. So it is a team's responsibility to gather the evidence, implement best practice, and make decisions. It is a team's responsibility that the communication is optimal and that there are no conflicts. Focusing on the [bachelor-educated] RN alone will not change the care practices." (Nursing scientist, North America, respondent 23)

Most experts (75\%) agreed that, ideally, BRNs and ANPs should work in a tandem. However, experts stressed that, as a starting point, more BRNs should be employed in the nursing homes: "Masters may indeed have more abstract thinking skills than bachelors. However, bachelors have more of these skills than the certified nurse assistants who now form the largest professional group in nursing homes. So the first step must be to involve more bachelor-educated registered nurses, and I certainly expect that they will make a difference." (Nursing scientist, Europe, respondent 4)

\section{DISCUSSION}

In this study, experts from different countries reached consensus on 16 competencies which in the future would be desirable for BRNs working in nursing homes. The identification of these competencies is an important step towards ensuring that the training of BRNs equips them with the competencies they will need to meet future demands. In every phase of our consensus exercise, most of the competencies identified were related to leadership and coaching, whereas traditionally more attention is paid to the development of expert knowledge and skills i.e. competencies related to nursing expertise and technical nursing skills.

Our findings are in line with the work from a collaboration between the Hartford Institute for Geriatric Nursing, the Pioneer Network and the Coalition of Geriatric Nursing Organizations. ${ }^{23}$ This collaboration reported that competencies beyond those traditionally associated with the nurse expert role would be needed to facilitate persondirected care and culture change in nursing homes. This was a US-based study whose objective was to identify nurse competencies which would facilitate culture change in nursing homes, ${ }^{23}$ whereas our study was broadened to consider the international context and competencies which should distinguish BRNs from other nursing staff working in nursing homes in the future.

In both survey rounds response rates were high (86\% and 78\%) and in their comments the experts stressed the importance of our consensus exercise; nevertheless the findings of our study should be interpreted carefully in view of some limitations of the research. Although the experts were selected carefully their opinions may not be representative. Another group of experts might have emphasized different competencies. As with all expert forecasts, there is a degree of uncertainty attached to their opinions and predictions. The extent to which the duties of BRNs of the future will 
require the competencies identified by the experts will depend on how the nursing homes of the future actually function. The implications of between-country differences in BRN training and the nursing home context were not considered. Within-country differences between nursing home contexts (e.g. small-scaled living vs. 'regular' ward or nursing unit) were not taken into account either. However, we believe that the overarching competencies identified in our consensus study are relevant to all nursing home contexts and that in the first instance it is important to focus on such general competencies.

When making staffing decisions, nursing home administrators should allocate BRNs in a way that allows them to act in a liaison role among nursing home residents, family members and the multidisciplinary nursing home staff and to serve as role models for other staff. Currently BRNs in nursing homes often carry out care tasks that could be delegated to other members of the team or fulfill management positions; these duties do not reflect the competencies which distinguish them from other nursing staff. Management support will be crucial to changing staffing practices. After further elaboration the BRN competencies identified in this research could be included in nursing home job profiles in many countries, paying particular attention to the specific nursing home context.

Our findings also have implications for development of BRN curricula and training programs in many countries. In the future particular attention should be paid to leadership and coaching competencies, which are not explicitly covered in most training programs at present. In addition, our findings also suggest that competencies related to evidence-based practice, communication and client assessment and geriatric expertise will be important. Most BRN training programs already pay attention to the development of competencies related to evidence-based practice and communication, but more attention should be paid to ensuring that BRNs have opportunities to acquire geriatric expertise. The findings of this study should be considered when updating existing BRN competency profiles or developing new ones. Our list of desirable distinguishing competencies provides a starting point for national level discussion with diverse stakeholders about which BRN competencies will be relevant in the future.

\section{CONCLUSION}

An important finding was that BRN competencies other than those traditionally associated with the nurse expert role were considered important. Paying more attention to these competencies might lead to improvements in nursing home care. For example, enabling BRNs to liaise between nursing home residents, family members and the multidisciplinary nursing home staff might lead to better collaboration and more person-centered care. Explicitly promoting BRNs as role models for other staff might 
help other nursing staff deal more effectively with, for example, more challenging resident behavior or new technological innovations.

Although there is continuing uncertainty about the future of nursing homes, our findings suggest that revision of current nursing curricula, nurse training programs and nursing home job profiles might be needed to meet the medically and psychologically complex needs of nursing home residents. 


\section{DISTINGUISHING COMPETENCIES OF BACCALAUREATE-EDUCATED RNS}

\section{REFERENCES}

1. Hasson H, Arnetz JE. Nursing staff competence, work strain, stress and satisfaction in elderly care: a comparison of home-based care and nursing homes. J Clin Nurs. 2008;17:468-481.

2. Katz PR. An international perspective on long term care: Focus on nursing homes. J Am Med Dir Assoc. 2011;12:487-492e1.

3. Rantz MJ, Skubic M, Alexander G, et al. Improving nurse care coordination with technology. Comput Inform Nurs. 2010;28:325-332.

4. Thoma-Lürken T, Bleijlevens MHC, Lexis MAS, Hamers JPH, De Witte LP. An overview of potential laborsaving and quality-improving innovations in long-term care for older people. J Am Med Dir Assoc. 2015;16:482-489.

5. Sloane PD, Zimmerman S, D'Souza MF. What will long-term care be like in 2040? N C Med J. 2014;75:326-330.

6. Arling G, Tu W, Stump TE, Rosenman MB, Counsell SR, Callahan CM. Impact of dementia on payments for long-term and acute care in an elderly cohort. Med Care. 2013;51:575-581.

7. Maly MB, Lawrence S, Jordan MK, et al. Prioritizing partners across the continuum. J Am Med Dir Assoc. 2012;13:811-816.

8. Sanford AM, Orrell M, Tolson D, et al. An International Definition for "Nursing Home". J Am Med Dir Assoc. 2015;16:181-184.

9. McLellan RK, Sherman B, Loeppke RR, et al. Optimizing health care delivery by integrating workplaces, homes, and communities: how occupational and environmental medicine can serve as a vital connecting link between accountable care organizations and the patient-centered medical home. J Occup Env Med. 2012;54:504-512.

10. Verbeek H, Van Rossum E, Zwakhalen SM, Ambergen T, Kempen GIJM, Hamers JPH. The effects of smallscale, homelike facilities for older people with dementia on residents, family caregivers and staff: design of a longitudinal, quasi-experimental study. BMC Geriatr. 2009;9:3.

11. Aiken LH, Sloane DM, Bruyneel L, et al. Nurse staffing and education and hospital mortality in nine European countries: a retrospective observational study. Lancet. 2014;383:1824-1830.

12. Spilsbury K, Hewitt C, Stirk L, Bowman C. The relationship between nurse staffing and quality of care in nursing homes: A systematic review. Int J Nurs Stud. 2011;48:732-750.

13. Backhaus R, Verbeek H, Van Rossum E, Capezuti E, Hamers JPH. Nurse staffing impact on quality of care in nursing homes: A systematic review of longitudinal studies. J Am Med Dir Assoc. 2014;15:383-393.

14. Weech-Maldonado R, Meret-Hanke L, Neff MC, Mor V. Nurse staffing patterns and quality of care in nursing homes. Health Care Manage Rev. 2004;29:107-116.

15. Van Eeden K, Moeke D, Bekker R. Care on demand in nursing homes: A queueing theoretic approach. Health Care Manag Sci. 2016;19:227-240.

16. McEwen M, White MJ, Pullis BR, Krawtz S. Essential content in RN-BSN programs. J Prof Nurs. 2014;30:333-340.

17. American Association of Colleges of Nursing. Essentials of baccalaureate education for professional nursing practice. Washington, DC: AACN; 2008. Available from: http://www.aacn.nche.edu/educationresources/BaccEssentials08.pdf.

18. American Association of Colleges of Nursing/Hartford Institute for Geriatric Nursing. Recommended Baccalaureate Competencies and Curricular Guidelines for the Nursing Care of Older Adults. Washington, DC: AACN; 2010.

Available from: http://www.aacn.nche.edu/geriatric-nursing/AACN_Gerocompetencies.pdf.

19. McGilton $\mathrm{KS}$, Heath $\mathrm{H}, \mathrm{Chu} \mathrm{CH}$, et al. Moving the agenda forward: a person-centered framework in longterm care. Int J Older People Nurs. 2012;7:303-309.

20. Stuurgroep HBO-VGG. Competentieprofiel HBO Verpleegkundige Gerontologie-Geriatrie (HBO-VGG). Waalwijk: Stuurgroep HBO-VGG; 2012.

Available from: http://www.hbo-vgg.net/download/file:competentieprofiel-hbo-vgg-juni2012.htm. 
21. Deutscher Pflegerat e.V. (DPR, German Nurse Council)/Deutsche Gesellschaft für Pflegewissenschaft e.V. (DGP, German Society of Nursing Science). Berlin/Duisburg: DPR/DGP; 2014. Available from: http://www.deutscher-pflegerat.de/presse/pressemitteilungen/2014-12-09_PP_dpr_dgp_arbeitsfelderakademisch-ausgebildeter-pflegefachpersonen_08-14.pdf.

22. Traynor $V$, Inoue $K$, Crookes $P$. Literature review: understanding nurse competence in dementia care. J Clin Nurs. 2011;20:1948-1960.

23. Mueller C, Burger S, Rader J, Carter D. Nurse competencies for person-directed care in nursing homes. Geriatr Nurs. 2013;34:101-104.

24. Tolson D, Rolland Y, Andrieu S, et al. International Association of Gerontology and Geriatrics: A global agenda for clinical research and quality of care in nursing homes. J Am Med Dir Assoc. 2011;12:184-189.

25. Eskes AM, Maaskant JM, Holloway S, et al. Competencies of specialised wound care nurses: a European Delphi study. Int Wound J. 2014;11:665-674.

26. Boerner KE, Coulombe JA, Corkum P. Core competencies for health professionals' training in pediatric behavioral sleep care: A Delphi study. Behav Sleep Med. 2015;13:265-284. 


\title{
CHAPTER 7
}

\author{
BLAZING A TRAIL FOR \\ BACCALAUREATE-EDUCATED \\ REGISTERED NURSES IN \\ NURSING HOMES: \\ EXPERIENCES OF ADMINISTRATORS \\ AND NURSING STAFF
}

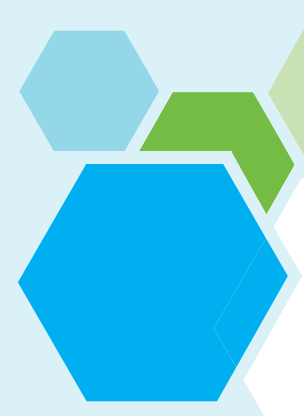

This chapter was submitted for publication as:

Backhaus R, Verbeek H, Van Rossum E, Capezuti E, Hamers JPH. Blazing a Trail for Baccalaureate-Educated Registered Nurses in Nursing Homes : Experiences of Administrators and Nursing Staff. 


\section{ABSTRACT}

Aims: To learn how organizations employ baccalaureate-educated registered nurses (BRNs) in nursing homes and the perceived added value in care practices those organizations enjoy as a result of these nurses' employment.

Background: Although providing care for nursing home residents is complex and thus requires a high level of skill, organizations often struggle to recruit and retain BRNs. Some nursing home organizations do not employ BRNs at all. Among those that do, it is unknown how well these organizations make use of BRNs' expertise or if their roles are different from those of other staff.

Design: A content analysis of 26 individual and three group interviews was conducted in the Netherlands.

Methods: Interviews were held at the board-, management- and staff-level in six nursing home organizations. Data were collected between January 2016 and May 2016.

Results: Organizations that employ BRNs had different understandings of the role and positioning of nurses than those organizations that did not employ them. Organizations that employ BRNs reported fewer difficulties attracting them than those that did not. Nurses were employed differently within and between organizations. Nurses experienced a variety of difficulties as they transitioned into their new roles in nursing homes, including a lack of role clarity, challenges with role naming, inadequate support, reticence from direct care teams, and barriers that arose due to their own behavior. The added value of BRNs perceived by respondents differed between and within organizations.

Conclusion: Our findings suggest that there is no "one size fits all" approach to employing BRNs in nursing homes. To ensure the satisfaction of both BRNs and the organizations that employ them, careful implementation and evaluation of their role is crucial. 


\section{INTRODUCTION}

Despite the heterogeneity in nursing homes worldwide, ${ }^{1}$ there are some common trends regarding care provision. Nursing homes in most countries will need to address a rising demand for services, more complex resident needs and an increase in patient flow in the near future. In addition, working in nursing homes is becoming increasingly complex due to a focus on resident-centered care, technological innovations such as robotic care and electronic monitoring systems, and the expectation for nursing home employees to partner with residents and their families throughout the care delivery process. ${ }^{2}$ Above all, nursing homes must ensure a high quality of care (QoC), an important component of which is the uptake of evidence-based practices. ${ }^{3}$ Meeting these new demands while maintaining QoC requires capable, highly skilled direct care staff. $^{2,4,5}$ Baccalaureate-educated registered nurses (BRNs) are educated with the very critical thinking skills needed to tackle the challenge of complex resident care needs. Evidence from the hospital sector suggests that an increase in BRNs employed in hospitals leads to better QoC for patients. ${ }^{6}$ However, for the nursing home sector, this relationship is less clear. ${ }^{7,8}$ Recent research concluded that too few BRNs are working in Dutch nursing homes to ascertain a relationship with QoC. ${ }^{9}$ Indeed, in most countries, few RNs are involved in direct resident care and BRNs are an especially scarce resource in nursing homes. ${ }^{2,10}$

Difficulties recruiting and retaining BRNs in nursing homes may be due to the low status and salary typically ascribed to those working in this setting. ${ }^{11,12}$ To guarantee sufficient staff to deliver around-the-clock direct care, some nursing home organizations opt to recruit a greater number of lower-educated (and therefore lower paid) staff. In the US, this practice has led to a high proportion of licensed practical/vocational nurses compared to RNs in many nursing homes. In the Netherlands, there are more certified nurse assistants (CNAs) than RNs employed in nursing homes, but these CNAs have two to three years of vocational training that renders them comparable to the licensed practical/vocational nurses in the US. ${ }^{13}$ As a result, the roles of BRNs and lowereducated staff have become blurred and tasks are often allocated inappropriately, as was demonstrated in recent observations conducted in Dutch nursing homes. ${ }^{14}$ In organizations where BRNs have the same roles as CNAs, their expertise might become invisible. ${ }^{12}$ If their added value and expertise is not recognized in an organization, the ratios of BRNs might decrease even further, as lower-educated staff come to be seen as capable of fulfilling the same duties as the higher-earning BRNs. ${ }^{12}$ Therefore, it is important that the role of BRNs be differentiated from that of other professionals working in direct care (e.g., RNs, CNAs) and that each can work to their full scope of practice. $^{15}$ 


\section{Background}

Evidence from the hospital sector suggests that an increase in BRNs leads to higher QoC. ${ }^{6}$ For the nursing home sector, findings concerning this relationship are inconsistent for three potential reasons. ${ }^{7,8}$ First, compared to the hospital sector, few BRNs are working in direct resident care in the nursing home sector and, in general, direct care staff in nursing homes tend to be less educated. ${ }^{7}$ In the Netherlands, direct care staff include (B)RNs (4 years of training), CNAs (2-3 years of training), nurse assistants ( 2 years of training), nurse aides (0.5-1 year of training) and untrained staff. ${ }^{16}$ Even though a specific educational program was established at different universities of applied sciences throughout the Netherlands offering BRNs the opportunity to specialize in gerontology and geriatrics, BRNs remain scarce in Dutch nursing homes. ${ }^{10}$ In a recent study conducted in 95 Dutch long-term care facilities, it was found that BRNs were employed in nearly half of 282 participating wards. ${ }^{17}$ Second, research has yet to confirm whether nursing homes that do employ BRNs make use of their expertise, or differentiate their roles from those of other staff members such as nursing home medical specialists, speech therapists, physiotherapists, and psychologists. ${ }^{18}$ Third, a BRN who is employed in a nursing home typically works for several different wards, meaning that the time he or she spends in any one ward is often low. ${ }^{17}$ The relationship between QoC and BRN employment could be mediated by this practice of assigning BRNs to too wide a range of wards. Therefore, more information is needed regarding the ways in which nursing home organizations employ BRNs and what added value they perceive their work to have for resident care and the organization as a whole. These insights into the successful recruitment and retention of BRNs in some organizations may also help explain why other organizations hesitate to employ, or are unable to attract, BRNs.

\section{THE STUDY}

\section{Aim}

The aim of the study was to obtain insights into the ways organizations employ BRNs in nursing homes and the added value in care practices that these organizations believe their BRNs contribute. To meet this aim, we asked three research questions:

1. How are BRNs employed as professional leaders in nursing homes?

2. How did administrators and staff of nursing home organizations perceive the implementation of the BRN role?

3. How do administrators and staff of nursing home organizations perceive the added value in care practices of BRNs in nursing homes? 
Table 1: Interviewed participants per organization

\begin{tabular}{|c|c|c|}
\hline Organization & Interviewed participants per & Respondent number(s) \\
\hline \multirow[t]{8}{*}{ Organization A } & Individual interviews: & \\
\hline & - 1 board member & 4 \\
\hline & - 2 ward managers & 5,6 \\
\hline & -2 BRNs & 12,13 \\
\hline & Group interview: & \\
\hline & - 2 vocationally-trained RNs & 7,8 \\
\hline & - 1 CNA & 9 \\
\hline & - 2 nurse assistants & 10,11 \\
\hline \multirow[t]{8}{*}{ Organization B } & Individual interviews: & \\
\hline & - 1 board member & 22 \\
\hline & - 1 director & 21 \\
\hline & -2 ward managers & 23,24 \\
\hline & -2 BRNs & 30,31 \\
\hline & Group interview: & \\
\hline & - 4 CNAs & $25,26,27,28$ \\
\hline & - 1 nurse assistant & 29 \\
\hline \multirow[t]{8}{*}{ Organization C } & Individual interviews: & \\
\hline & - 1 board member & 32 \\
\hline & - 2 location managers & 33,34 \\
\hline & -2 BRNs & 39,40 \\
\hline & Group interview: & \\
\hline & - 1 vocationally-trained RN & 35 \\
\hline & - 1 CNA & 36 \\
\hline & - 2 nurse assistants & 37,38 \\
\hline \multirow[t]{3}{*}{ Organization D } & Individual interviews: & \\
\hline & - 1 director & 1 \\
\hline & - 2 ward managers & 2,3 \\
\hline \multirow[t]{3}{*}{ Organization E } & Individual interviews: & \\
\hline & - 1 board member & 19 \\
\hline & - 2 ward managers & 18,20 \\
\hline \multirow[t]{4}{*}{ Organization F } & Individual interviews: & \\
\hline & - 1 board member & 14 \\
\hline & - 1 director & 17 \\
\hline & - 2 ward managers & 15,16 \\
\hline
\end{tabular}




\section{Design}

Qualitative interviews were conducted between January 2016 and May 2016 using a content analysis design. ${ }^{19}$

\section{Participants}

Participants were from six nursing home organizations in the Netherlands. Nursing homes in the Netherlands provide long-term residential care for individuals with dementia (referred to as psychogeriatric) or physical disabilities (referred to as somatic), as well as short-term skilled care for rehabilitation of subacute conditions. ${ }^{18}$ We used purposive sampling to select participants who were expected to provide particularly rich information. ${ }^{19}$ These participants were drawn from two different "types" of organizations. One type were those that were nationally regarded as on the forefront of employing BRNs within their nursing homes. The other type were organizations that were shown in a previous study to have no or very few BRNs employed in their nursing homes. To ensure that the collected data represented a range of perspectives, we aimed to include participants from different organizational levels. In organizations that did employ BRNs, we invited board members, ward/location managers, staff members from direct care teams (including vocationally-trained RNs and CNAs) and BRNs themselves to participate. In organizations that did not employ BRNs, we invited board members and ward/location managers to participate. Two organizations (organizations $B$ and $F$ ) proposed to interview a director as well, as they were expected to provide richer information on the research topic. In a third organization (organization D), the director participated instead of a board member. See Table 1 for a detailing of the roles held by the individuals interviewed from each organization.

\section{Data collection}

Data were collected via semi-structured, face-to-face individual or group interviews conducted between January 2016 and May 2016. Board members, directors, managers, and BRNs were interviewed individually, while direct care staff members participated in group interviews. A topic list was used to structure the interviews (see Table 2). Interviews were held in a quiet room in the nursing homes. All interviews were conducted by the primary author. During group interviews, a second researcher was present to take notes and to verify that all interview topics were covered. 
Table 2: Interview topic list

\begin{tabular}{|c|c|c|}
\hline & $\begin{array}{l}\text { Organizations that } \\
\text { employ BRNs in } \\
\text { nursing homes }\end{array}$ & $\begin{array}{l}\text { Organizations that do } \\
\text { not or hesitate to } \\
\text { employ BRNs in } \\
\text { nursing homes }\end{array}$ \\
\hline \multicolumn{3}{|l|}{ Board members and directors } \\
\hline Vision of the employment of BRNs in nursing homes & $\checkmark$ & $\checkmark$ \\
\hline Experienced added value of BRNs in nursing homes & $\checkmark$ & - \\
\hline \multicolumn{3}{|l|}{ Ward/location managers } \\
\hline Vision of the employment of BRNs in nursing homes & $\checkmark$ & $\checkmark$ \\
\hline Positioning of BRNs in nursing homes & $\checkmark$ & - \\
\hline Implementation of BRN role in nursing homes & $\checkmark$ & - \\
\hline Experienced added value of BRNs in nursing homes & $\checkmark$ & - \\
\hline \multicolumn{3}{|l|}{ Direct care staff members } \\
\hline Experienced added value of BRNs in nursing homes & $\checkmark$ & - \\
\hline \multicolumn{3}{|l|}{ BRNs } \\
\hline Positioning of BRNs in nursing homes & $\checkmark$ & - \\
\hline Implementation of BRN role in nursing homes & $\checkmark$ & - \\
\hline Experienced added value of BRNs in nursing homes & $\checkmark$ & - \\
\hline
\end{tabular}

\section{Ethical considerations}

According to Dutch law, approval from an ethics committee was not needed, as no patients were involved in the study and the data collection was not burdensome (http://www.ccmo.nl/en/your-research-does-it-fall-under-the-wmo). Consent for participation and audiotaping was obtained prior to all interviews. Data were analyzed anonymously.

\section{Data analysis}

All interviews were audiotaped and transcribed verbatim by the primary author. Transcripts were read twice each before being analyzed using directed content analysis. $^{20}$ The primary author selected four interviews to analyze using a four-step approach that would determine the codes for the remaining transcripts. First, the text of all four transcripts was divided into "meaning units". ${ }^{21}$ Meaning units could consist of as little as one sentence or as much as entire paragraphs of a transcript. Second, all meaning units were condensed into short descriptions of the text (manifest content) and, when possible, interpretations of their underlying meanings (latent content) were documented. ${ }^{21}$ Third, the condensed meaning units were coded. Topics from the interview topic list were used as the basis for the initial codes. ${ }^{22}$ Text passages that 
could not be coded with the initial codes were given a new code. In the fourth and final step, depending on the breadth of a code, subsequent analyses were conducted to identify subcodes. ${ }^{20,22}$ To ensure quality of the analysis, two members of the research team separately analyzed these same four interviews using the same four-step approach. Their analyses were compared with those of the primary author. Consensus on small differences in the condensed meaning units and in coding was reached through discussion. It was concluded that the codes did not need to be changed. After that, all other interviews were analyzed by the primary author and all analyses were discussed with the research team. Based on the analyses of all interviews, the research team concluded that data saturation was reached, as no new themes emerged in the data.

\section{Validity and reliability/Rigour}

To enhance the quality and integrity of our study, different strategies were applied. Purposive sampling enabled us to continuously compare the views of different respondent groups (i.e., board members, managers, direct care staff, and BRNs) during the data analysis and to illuminate differences between these groups. ${ }^{23}$ Person and space triangulation enabled us to capture more complete insights into the issues under study. Person triangulation refers to the collection of data from different respondent groups, aimed at validating data by including multiple perspectives. ${ }^{24}$ Space triangulation refers to collecting data at multiple sites to test for cross-site consistency. ${ }^{24}$ Cross checking of the coding process by members of the research team led to a further refinement of the coding frame. ${ }^{23}$

\section{FINDINGS}

In this study, 26 individual interviews and three group interviews (with 14 participants in total) were held with staff members from six nursing home organizations. Table 3 provides insights into respondents' demographic and occupational characteristics. In the following three sections, the results of the interviews are presented as they apply to the three research questions. 
Table 3: Respondents' characteristics

\begin{tabular}{|c|c|c|}
\hline & $\begin{array}{l}\text { Organizations that } \\
\text { employ BRNs in } \\
\text { nursing homes }\end{array}$ & $\begin{array}{l}\text { Organizations that do } \\
\text { not or hesitate to } \\
\text { employ BRNs in } \\
\text { nursing homes }\end{array}$ \\
\hline Board members and directors $(n=8)$ & $(n=4)$ & $(n=4)$ \\
\hline Age (mean/range) & $58(55-64)$ & $55(43-60)$ \\
\hline Gender: Female & 2 & 2 \\
\hline Years of experience in current position (mean/range) & $12(8-17)$ & $3(0-8)$ \\
\hline RN background (Yes) & 3 & 1 \\
\hline Ward/location managers $(n=12)$ & $(n=6)$ & $(n=6)$ \\
\hline Age (mean/range) & $53(43-59)$ & $49(42-56)$ \\
\hline Gender: Female & 4 & 5 \\
\hline Years of experience in current position (mean/range) & $9(0-24)$ & $4(0-16)$ \\
\hline RN background (Yes) & 6 & 3 \\
\hline Direct care staff members $(n=14)$ & $(n=14)$ & \\
\hline Vocationally-trained RNs & 3 & \\
\hline CNAs & 6 & \\
\hline Nurse assistants & 5 & \\
\hline Age (mean/range) & $41(25-62)$ & \\
\hline Gender: Female & 14 & \\
\hline Years of experience in current position (mean/range) & $13(0-31)$ & \\
\hline $\operatorname{BRNs}(n=6)$ & $(n=6)$ & \\
\hline Age (mean/range) & $39(30-49)$ & \\
\hline Gender: Female & 6 & \\
\hline Years of experience in current position (mean/range) & $3(2-7)$ & \\
\hline
\end{tabular}

\section{Employing BRNs as professional leaders in nursing homes}

Interview data showed that, within and between the three participating organizations that employ BRNs within their nursing homes, there is great variation in the positioning, roles, tasks and responsibilities of BRNs (see Table 4). For example, BRNs in some organizations held formal positions within a direct care team, while in other organizations, they were positioned supernumerary to the teams (as institutional level consultants). BRNs were also expected to improve QoC in two different ways: some were instructed to focus on their specific areas of expertise (e.g., resident behavior), while others sought to improve QoC in general. 


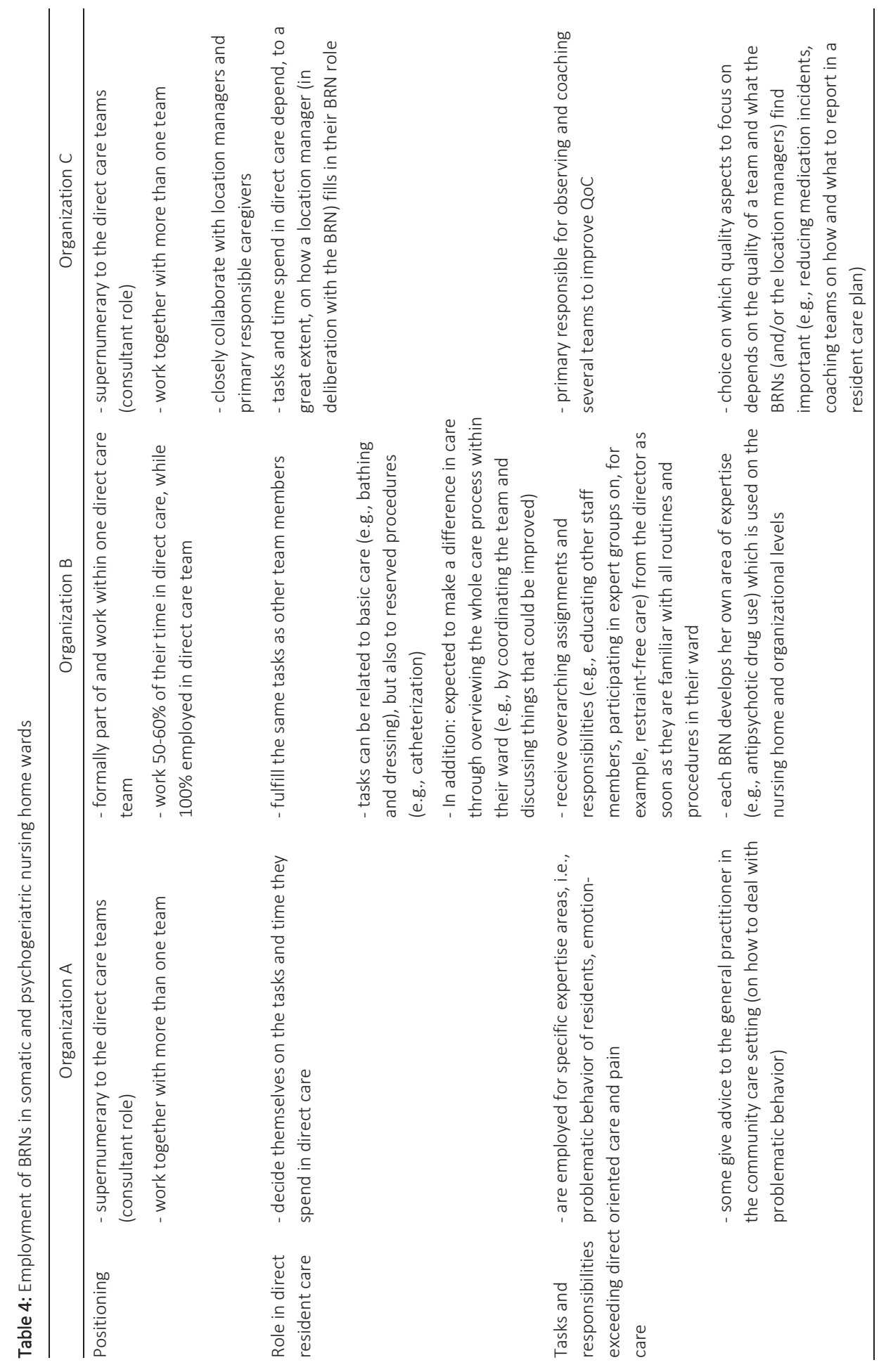


Although all nursing home board members shared the opinion that employing BRNs within their nursing home would be of added value, not all organizations actively employed them. Organizations that did employ BRNs in their nursing homes differed in their vision of how to position the BRNs in nursing homes, took responsibility for employing them, and reported fewer problems enticing them to work in their nursing homes.

Having a vision of BRNs in nursing homes

Board members of organizations that employ BRNs had a vision of how to utilize their skills to improve QoC. At the ward level, they expected BRNs to fulfill an informal, clinical leadership role for direct care teams. In their opinion, BRNs should serve as role models for direct care staff and help teams implement care innovations such as a new electronic resident record for each unit, as just one example. At the organizational level, BRNs were expected to advise managers, participate in working groups, or represent their organization in external meetings. All three board members preferred employing BRNs that received specialized training in gerontology and geriatrics. They mentioned that having a vision for BRNs within nursing homes is crucial for their successful employment:

If a board member has no vision on it, yes, then it is doomed. Because then you do it because your neighbor does. And that happens often. (Board member, organization C, respondent 32)

Board members of organizations that do not employ BRNs did not have a vision of how they might utilize them in their nursing homes and could not describe how they would employ them within their organization.

\section{Taking responsibility and making room for employing BRNs in nursing homes}

In the organizations that did not employ BRNs already, nobody felt primarily responsible for recruiting BRNs. Board members and managers felt partially responsible, but, at the same time, felt that the other party should take more responsibility. Board members stressed the lack of specific guidelines on this issue, and concluded that the decision to employ BRNs within nursing homes ultimately rested with the managers. According to board members, managers often opted to hire lower educated staff who could be paid less. At the same time, managers felt that the board members did not adequately support employment of BRNs within their nursing homes. For example, the managers highlighted that the board members did not facilitate additional education so that their vocationally trained RNs could become BRNs.

In one organization that does not regularly employ BRNs, respondents felt that it was difficult to "make room" for more highly educated staff. By contrast, in the organizations that do employ BRNs, board members felt that it was their responsibility to actively facilitate the employment of BRNs: 
By indicating that I find it important. That I stand behind it. And yet, even at that point, despite self-organizing teams, to outline a number of guidelines and to say, 'Still, I want to see that at least in every ward one or two baccalaureate-educated people are walking around.' And there is nothing wrong with it. And, for example, through giving our people the opportunity to follow a BRN educational program, which is facilitated by the company. (Board member, organization $\mathrm{B}$, respondent 22)

\section{Attracting BRNs to work in nursing homes}

The organizations that employ BRNs did not report that they have any problems recruiting or retaining them. By contrast, board members in organizations that did not employ BRNs mentioned that they experienced difficulties attracting BRNs to work in their nursing homes. For example, they reported that students who did their internships within the organization preferred to work in a hospital after graduation. Most of the managers from organizations that do not employ BRNs believed the work in somatic or psychogeriatric long-term nursing home wards lacks the complexity needed to attract BRNs.

\section{Implementing the $B R N$ role}

Most interviewed BRNs experienced difficulties when they started working in the nursing home where they were employed. These difficulties were related to role clarity, the naming of the BRN role, the extent to which they received support, the openness of the direct care teams, and how they were expected to work within the nursing team.

Role clarity

As the BRN role was a new one in the participating organizations, clear job descriptions were lacking. Most BRNs stressed that, especially in the beginning, they missed guidelines on how they should perform their role:

Sometimes I still have the feeling, 'Gosh, provide me with some guidelines or certainty.' Then you think, 'Am I now doing the work of a policy employee or of a manager? Or...?' That is always a consideration within myself, too, like, 'Am I doing my work well?' (BRN, organization C, respondent 39)

Board members in organizations that employed BRNs agreed that role clarity was a prerequisite for the successful implementation of the BRN role in nursing homes. Within their organizations, the roles and tasks BRNs fulfilled were differentiated from those that other staff members fulfilled. Role clarity received continuous attention from the board members of these organizations that employed BRNs. Despite this attention to clarifying their role, some of the BRNs felt they had to overcome resistance and win trust from coworkers, who believed the BRNs were taking over part of their role. 
Naming of the BRN role

Different organizations named the BRN role differently within their nursing homes. In two of the organizations that employ BRNs, respondents talked about "BRNs" or "BRNGGs" (referring to a BRN specialized in gerontology and geriatrics). In one organization, respondents used the term "behavior nurse" (i.e., a nurse that focuses on challenging dementia-related behavior) when talking about the BRNs. According to some respondents, referring to a BRN as "the BRN" might be problematic, as it could establish an unnecessary sense of hierarchy. By contrast, referring to a BRN as a "behavior nurse" provides more information about the specific role of the BRN within the organization. Thus, some respondents stressed that the name used to refer to BRNs within an organization can help make their role clearer.

\section{Support}

Most interviewed BRNs mentioned that, especially in the beginning, they felt it was important to have the opportunity to talk with other BRNs. In all organizations, BRNs working in nursing homes had close contact with one another. In one organization, the BRNs had formal meetings in which they could discuss the difficulties they were encountering. Some respondents stressed the importance of shadowing other BRNs when they first started working in a nursing home, to see how they had implemented their role.

In addition, BRNs reported that it was helpful to discuss difficulties with their managers. Similarly, the interviewed managers felt that it was important to support the BRNs:

It took some time before it was clear for us what the BRN was going to do. Like, 'That well, that not.' But one of the conditions, for me, was also that I sat together with her every week, like, 'Okay, what are you doing at the moment?' Etc. At one point she had too much on her plate. Well, setting priorities, requires also a bit of coaching from me towards her, I find that important. (Location manager, organization $\mathrm{C}$, respondent 34)

Nevertheless, the interviewed managers felt that not all managers within their organization were open to the employment of BRNs within their wards/locations:

You can see that managers agree with it or not. I expected that. Because you will come very close into such a team and in the beginning, there was also resistance, and I expected that to happen. [...] Because you get an inside look. And not everyone is open for that. Because sometimes you just have your things not properly regulated. (Ward manager, organization A, respondent 5) 
Direct care team openness

Not all BRNs experienced challenges when they started working in their nursing homes. Whether or not BRNs experienced difficulties also depended on the openness of their team. Teams that were open to change and were committed to improving QoC were not resistant to the addition of BRNs.

The behavior of the BRN within the direct care team

Whether or not BRNs experienced difficulties when they started working within the nursing home also partly depended on how the BRNs behaved when working with other staff members. Some BRNs mentioned that the direct care staff they worked with should not get the feeling that the BRNs stood "above" them, and they had to make efforts to be viewed as part of the team:

I have worked in direct resident care in a ward [...] and someone said: 'But you are the 'behavior consultant.' And I said: 'But I am also a nurse, and I will stay being a nurse at all times.' And then you notice that barriers disappear, because you are one of them, thus you will have much easier access. [...] I always say: "I find it very important that the BRN does not think, "I am now the BRN, I do my coordination tasks and you wash the residents and I tell you what you have to do." I think working in direct resident care is not an inferior task. As a nurse, that is part of your job, too. And I also like to do it. (BRN, organization A, respondent 12)

Some of the interviewed managers said that it is the role of a manager to monitor the BRN and ensure he or she does not attain a "lofty" position within the direct care teams.

\section{Perceived added value in care practice}

The added value of BRNs perceived by board- and management-level respondents differed from the added value perceived by direct care staff. Therefore, we distinguish between the experiences of board members, nursing home directors and ward/location managers, and the experiences of direct care staff members.

Experiences of board members, directors and ward/location managers

Board- and management-level respondents unanimously stressed that BRNs had added value for their nursing homes. Depending on how the BRNs were positioned within the organization, their perceived added value differed. In all organizations, respondents felt that the employment of BRNs was of added value for the way in which care was delivered, and that BRNs had positive effects on residents and other employees. Respondents felt that employing a BRN who serves as a coach for direct care teams and is consistently present in the ward improved the delivery of care. Having a BRN fill this 
coaching role meant that issues in the ward could be discussed, reflected upon, and directly addressed.

Depending on how the BRNs were positioned in the different nursing homes, board members and managers mentioned a range of positive resident outcomes, such as a decrease in the use of physical restraints. However, resident-level outcomes were not always easily measurable, as they demand conscious reflection on the BRN's way of working:

The effect that it has to look together at the client, 'What do you see? What do I see?' and that you talk about it. Taking five minutes of your time for that. To, together with the client, also to ask: 'I give you every morning black coffee, do you actually like that?' This effect has been enormous and has really given a boost to client-centeredness. (Board member, organization A, respondent 4)

On the employee level, board members from two organizations stressed that employing BRNs led to higher satisfaction among direct care staff. In one organization, it was added that the higher satisfaction led to a decrease in absenteeism. Different respondents had experienced that BRNs helped direct care staff to cope with the daily problems they experience in their practice.

Experiences of direct care staff members

The experiences of direct care staff members differed between organizations. When BRNs were employed in very specific areas of expertise (i.e., problematic resident behavior, pain), the interviewed direct care staff members who worked in psychogeriatric wards were very satisfied with the BRNs and perceived their added value:

But I see also, since we have her, changes in our ward, in the sense: we ourselves already take care of a good observation, but also through being more alert before calling in the behavior nurse. Indeed, also regarding pain, to, for example, already complete a pain-score list for a few days that we can hand over 'Look, this is what we have already seen.' (Vocationally-trained RN, organization A, respondent 8)

In somatic wards within that same organization, BRNs were seldom contacted for advice, as direct care staff considered the expertise of the BRNs (i.e., resident behavior, emotion-oriented care, pain) as less relevant for a somatic ward.

In the other two organizations, respondents were less positive regarding the addition of BRNs to their teams. In organization B, where BRNs are part of one direct care team, respondents reported that they did not experience any added value of the BRNs. The interviewed direct care staff members said they did not see the BRNs fulfilling the coordinating role they are expected to hold when working in direct resident 
care. However, these respondents stressed that the added value of a BRN sometimes depends on the individual BRN him- or herself:

But we have one BRN here, working in another ward, she is a topper. She also gives training sessions and does a lot of extra things. She also travels the whole country to see 'What is going on within your organization? What can we improve?' [...] I am sure that she is of added value. (CNA, organization B, respondent 25)

In organization $\mathrm{C}$, the interviewed respondents worked in a location that was taken over from a bankrupt organization half a year ago. In this location, the BRN role was new and respondents mentioned that they did not experience any added value of the BRNs since their role had not been made clear. These respondents stressed that their experiences might not be representative of those of other staff in the organization, as the role of BRNs had been further developed in other locations within their organization.

\section{DISCUSSION}

This study found that having a vision of how to utilize BRNs within nursing homes is a key factor in determining whether or not an organization employs BRNs in their nursing homes. Organizations that do not employ BRNs assume that BRNs do not want to work in nursing homes, while organizations that have a clear vision of how to implement their role are successful in employing and retaining BRNs. In organizations that employ BRNs, the percentage of board members and managers with an RN background was higher. Within and between the organizations that employ BRNs, there is great variation in the positioning, roles, tasks and responsibilities of BRNs. Difficulties BRNs experienced when they started working in the nursing homes were related to their role clarity, the framing of the BRN role, the extent to which they received support, the openness of the direct care teams, and their own behavior. The added value of BRNs perceived by direct care staff members differed between organizations.

Without clear guidelines from professional organizations and academic institutions to prepare BRNs for nursing home practice, individual organizations are left to independently determine how to employ BRNs within their organizations. The findings of this study suggest that the employment of BRNs within nursing homes requires board-level leadership, as board members are expected to have a vision of how to utilize BRNs within the organization and to actively facilitate their employment. In organizations lacking that vision, the role of the BRN is not distinguished from the roles of other staff. This helps to explain why these organizations experienced difficulties in attracting and retaining BRNs. None of the organizations that had a clear vision of how to employ BRNs experienced these difficulties. 
Even though our findings indicate that there is no "gold standard" for employing BRNs in nursing homes, they suggest different macro-, meso- and micro-level factors that could be considered when implementing the BRN role in nursing homes. On a macro-level, role clarity was seen as a prerequisite for the successful implementation of the BRN within the nursing home, which is closely related to having a vision of how to employ BRNs. McAiney et al., ${ }^{25}$ who evaluated a practice model for nurse practitioners (NPs) in long-term care homes, concluded that role clarity facilitated the implementation of the NP role, as it increased staff members' awareness of the knowledge and skills of NPs and led to better support from staff members. Respondents in our study stressed that it is necessary to inform and prepare staff members at all levels within the organization before implementing the BRN role, while paying attention to how the role is framed. Findings from McAiney et al. ${ }^{25}$ confirm that staff members should be engaged in the process of identifying the role that BRNs should fulfill within the organization before the BRN begins working there, as this process made the actual implementation of the NP role more successful in their study. Hospitals' experiences with implementing advanced practice nurse (APN) roles similarly suggest that, within organizations, consensus on role attributes (i.e., role scope, goals, core competencies, educational requirements) should be reached prior to role implementation. ${ }^{26}$

On a meso-level, BRNs in our study reported that they considered the support and openness of managers, direct care teams, and other BRNs to be important, as they expected a degree of resistance from other staff. Given the additional educational preparation in geriatrics and their role as consultants to other nurses and clinicians, the BRN role can be compared to the APN role in nursing homes. Barriers to the development of APN roles include physician resistance. ${ }^{27}$ Moreover, managers who were supportive when implementing the APN role, while at the same time trusting the APN and supporting them in implementing their role autonomously, were considered crucial for APN role achievement. ${ }^{26}$ Our results similarly suggest that support and openness of managers and direct care teams regarding role clarity for BRNs is an essential first step towards reducing resistance to their employment in nursing homes. In a recent study of the roles of BRNs who specialize in gerontology and geriatrics, Huizenga et al. ${ }^{10}$ concluded that these BRNs are pioneers who have few role models available to them, yet these same BRNs considered role modeling a prerequisite for good role development. While not every organization already employs BRNs that can serve as role models for new colleagues, our findings suggest that it might be wise for organizations interesting in hiring BRNs to start by employing at least two, since they could support each other and turn to one another for guidance. ${ }^{26}$ In addition, a virtual learning network among BRNs working in nursing homes might help BRNs develop their role.

On a micro-level, BRNs who work in nursing homes need to hone their communication skills so they can convey their affiliation with the other nurses and make clear that they are not filling a new role that is "above" that of others. Between 
organizations, opinions differed regarding whether BRNs should be formally part of a direct care team, or should only work together with these teams as institutional-level consultants. Based on the experiences of participants from two organizations, our findings suggest that one BRN working with several teams might be effective in improving QoC in nursing homes. Moreover, it might not be wise to employ a BRN within each ward. If a BRN is employed to work in direct resident care on a specific ward exclusively, he or she might develop close relationships with the team members. These close relationships might hinder the BRN's ability to fulfill an informal leadership role. By contrast, when working with too many teams, the time spent in any one ward would be low, potentially leading to an "out of sight, out of mind" mentality among the team members that could undercut the BRN's authority. ${ }^{25}$

BRNs working in the participating organizations were expected to improve QoC in two ways. BRNs either focused on improving QoC in their specific areas of expertise (e.g., residents' dementia-related behaviors) or on improving QoC in general. Both approaches were reported by participants as adding value to care practices; our findings do not suggest that one approach is more favorable than the other. Nevertheless, to use their unique competencies effectively and avoid unrealistic expectations, we suggest organizations provide focused direction for BRNs.

With regard to the perceived added value of BRNs in care practice, we found discrepancies between the experiences of board- and management-level participants and those of direct care staff members. In two of the three organizations, board members and managers seemed to be more satisfied with the BRNs than direct care staff. There were several potential reasons for this discrepancy. For one, board members and managers have a broader view of staff roles within their organization. In the process of implementing the BRN role, they likely considered how various staff impact resident, staff and organizational outcomes. It is possible that, during the interviews, they reflected on their ideas or expectations about the added value of BRNs instead of reporting their actual experiences.

In contrast, direct care staff members were not likely reflecting on the BRNs' potential to improve QoC in general, but instead reporting their actual experiences with the BRNs. In only one organization, the direct care staff members reported that the role of the BRNs was clear and that they experienced added value. In the organization where staff members reported that the BRNs performed the same tasks in direct care as they did, it might be the case that they did not believe the BRNs added any value, and were only differentiated by "a piece of paper." To ensure that organizations benefit from the work of BRNs, they should evaluate whether their staff members' perceptions of the BRN role align with the goals they hope to fulfill by employing BRNs. ${ }^{28}$ However, conducting well-planned evaluations of the effectiveness of BRNs is challenging. ${ }^{29}$ In our study, even though respondents reported that the employment of BRNs led to a decrease in physical restraint use or direct care staff absenteeism, it is possible that other factors led to these outcomes. 


\section{Limitations}

To our knowledge, this is the first study to explore how BRNs can be employed in nursing homes in a way that enhances QoC. Even though it was not our aim to provide a complete picture of all possible ways to employ BRNs, we acknowledge that the generalizability of our findings is limited due to the small sample size. Our data collection and analysis methods did not allow us to distinguish between male and female BRNs. However, our findings show that personal characteristics of BRNs might have an influence on how they fulfill their role. The gender of the BRN might be one of these characteristics.

\section{CONCLUSION}

This exploratory, qualitative study demonstrates that there is no "gold standard" for employing BRNs in nursing homes. Instead, it presents different factors that can contribute to the development of BRN roles in nursing homes. In addition, insights are provided into why some organizations succeed in employing BRNs in nursing homes, while other organizations do not.

The findings of this study should be used to inform future research. Future studies should aim to provide more insight into the tasks BRNs are actually performing in nursing homes, either through direct observations, or having BRNs complete logs or diaries where they track their activities. To inform policy, evaluations of the effectiveness (including cost) of employing BRNs in nursing homes should be conducted. However, it might be difficult to select generic outcome variables for these analyses.

Our findings suggest that BRNs might improve QoC in nursing homes and have different implications for practice. A careful implementation and evaluation of the BRN role seems crucial for their successful employment in nursing homes. The steps presented in the "participatory, evidence-based, patient-focused process, for guiding the development, implementation, and evaluation of advanced practice nursing" (PEPPA) framework ${ }^{30}$ may prove useful in guiding this process. 


\section{REFERENCES}

1. Sanford AM, Orrell M, Tolson D, et al. An international definition for "nursing home." J Am Med Dir Assoc. 2015;16:181-184.

2. Backhaus R, Verbeek H, Van Rossum E, Capezuti E, Hamers JPH. Future distinguishing competencies of baccalaureate-educated registered nurses in nursing homes. Geriatr Nurs. 2015;36:438-444.

3. Boström AM, Slaughter SE, Chojecki D, Estabrooks CA. What do we know about knowledge translation in the care of older adults? A scoping review. J Am Med Dir Assoc. 2012;13:210-219.

4. Katz PR. An international perspective on long term care: Focus on nursing homes. J Am Med Dir Assoc. 2011;12:487-492.

5. Sloane PD, Zimmerman S, D'Souza MF. What will long-term care be like in 2040? N C Med J. 2014;75:326-330.

6. Aiken LH, Sloane DM, Bruyneel L, et al. Nurse staffing and education and hospital mortality in nine European countries: A retrospective observational study. Lancet. 2014;383:1824-1830.

7. Spilsbury K, Hewitt C, Stirk L, Bowman C. The relationship between nurse staffing and quality of care in nursing homes: A systematic review. Int J Nurs Stud. 2011;48:732-750.

8. Backhaus R, Verbeek H, Van Rossum E, Capezuti E, Hamers JPH. Nurse staffing impact on quality of care in nursing homes: A systematic review of longitudinal studies. J Am Med Dir Assoc. 2014;15(6), 383-393.

9. Hamers JPH, Backhaus R, Beerens HC, Van Rossum E, Verbeek H. Meer is niet per se beter - De relatie tussen personele inzet en kwaliteit van zorg in verpleeghuizen. Maastricht: Datawyse Universiteire Pers Maastricht; 2016.

10. Huizenga $P$, Finnema $E$, Roodbol P. Learnt and perceived professional roles of a new type of nurse specialized in gerontology and geriatrics, a qualitative study. J Adv Nurs. 2016;72:1552-1566.

11. Eldh AC, Zijpp T, McMullan C, McCormack B, Seers K, Rycroft-Malone J. 'I have the world's best job' staff experience of the advantages of caring for older people. Scand J Caring Sci. 2015;30:365-373.

12. Phelan A, McCormack B. Exploring nursing expertise in residential care for older people: a mixed method study. J Adv Nurs. 2016;72:2524-2535.

13. Verkaik R, Francke AL, Van Meijel B, Spreeuwenberg PMM, Ribbe MW, Bensing JM. The introduction of a nursing guideline on depression at psychogeriatric nursing home wards: effects on certified nurse assistants. Int J Nurs Stud. 2011;48:710-719.

14. Tuinman A, De Greef MH, Krijnen WP, Nieweg RM, Roodbol PF. Examining time use of Dutch nursing staff in long-term institutional care: A time-motion study. J Am Med Dir Assoc. 2016;17:148-154

15. McGilton KS, Bowers BJ, Heath $\mathrm{H}$, et al. Recommendations from the international consortium on professional nursing practice in long-term care homes. J Am Med Dir Assoc. 2016;17:99-103.

16. Willemse B, Depla M, Smit D, Pot A. The relationship between small-scale nursing home care for people with dementia and staff's perceived job characteristics. Int Psychogeriatr. 2014;26:805-816.

17. Backhaus R, Van Rossum E, Verbeek H, et al. Relationship between RN staffing and quality of care in Dutch long-term care facilities. Gerontologist. 2015;55:806.

18. Huls M, De Rooij SE, Diepstraten A, Koopmans R, Helmich E. Learning to care for older patients: hospitals and nursing homes as learning environments. Med Educ. 2015;49:332-339.

19. Polit DF, Beck CT. Nursing research: Generating and assessing evidence for nursing practice. Philadelphia: Lippencott Williams \& Wilkins; 2008.

20. Hsieh HF, Shannon SE. Three approaches to qualitative content analysis. Qual Health Res. 2005;15:12771288.

21. Graneheim UH, Lundman B. Qualitative content analysis in nursing research: concepts, procedures and measures to achieve trustworthiness. Nurs Educ Today. 2004;24:105-112.

22. Utens CM, Dirksen CD, Van der Weijden T, Joore MA. How to integrate research evidence on patient preferences in pharmaceutical coverage decisions and clinical practice guidelines: a qualitative study among Dutch stakeholders. Health Policy. 2016;120:120-128. 
23. Barbour RS. Checklists for improving rigour in qualitative research: a case of the tail wagging the dog? Brit Med J. 2001;322:1115.

24. Polit DF, Beck CT. Nursing research: generating and assessing evidence for nursing practice. Philadelphia: Wolters Kluwer Health; 2017.

25. McAiney CA, Haughton D, Jennings J, Farr D, Hillier L, Morden P. A unique practice model for nurse practitioners in long-term care homes. J Adv Nurs. 2008;62:562-571.

26. Jokiniemi K, Haatainen K, Pietilä AM. From challenges to advanced practice registered nursing role development: qualitative interview study. Int J Nurs Pract. 2015;21:896-903.

27. Fougère B, Morley JE, Decavel F, et al. Development and implementation of the advanced practice nurse worldwide with an interest in geriatric care. J Am Med Dir Assoc. 2016;17:782-788.

28. Li J, Westbrook J, Callen J, Georgiou A, Braithwaite J. The impact of nurse practitioners on care delivery in the emergency department: a multiple perspectives qualitative study. BMC Health Serv Res. 2013;13:356-364.

29. Donald F, Martin-Misener R, Carter N, et al. A systematic review of the effectiveness of advanced practice nurses in long-term care. J Adv Nurs. 2013;69:2148-2161.

30. Bryant-Lukosius D, Dicenso A. A framework for the introduction and evaluation of advanced practice nursing roles. J Adv Nurs. 2004;48:530-540. 

CHAPTER 8

GENERAL DISCUSSION 

The aim of this dissertation was to examine direct nursing care staffing in nursing homes. Besides considering staffing levels, particular attention was paid to the competencies, tasks and employment of baccalaureate-educated registered nurses (BRNs), as they are expected to serve as informal leaders with the ability to lead improvements and redesign practice environments in nursing homes. More specifically, this dissertation aimed to provide insight into 1) the relationship between direct nursing care staffing and staff-related work environment characteristics and quality of care (QoC) in nursing homes; 2) future desirable distinguishing competencies of BRNs in nursing homes; and 3) how organizations employ BRNs in nursing homes and what is the added value they bring to practice. To meet these objectives, six studies were conducted (Chapters 2-7).

In this final chapter, the main findings of these studies are summarized, methodological and theoretical considerations are discussed, and future directions for practice, education, policy, and research are presented.

\section{MAIN FINDINGS}

In this dissertation, no consistent evidence was found for a positive relationship between direct nursing care staffing and QoC in nursing homes. In our systematic review, higher staffing levels were associated with both higher and lower QoC indicators. Moreover, major methodological weaknesses (e.g., timing of data collection, accuracy of staffing data) limited the interpretation of the results. In our cross-sectional studies, no consistent evidence was found for a relationship between a) the presence of BRNs and clinical QoC outcomes, and b) the number of direct nursing care staff to both clinical and staff-reported QoC outcomes. Clinical QoC outcomes in most wards were acceptable and, overall, staff members from both somatic and psychogeriatric wards were satisfied with the $\mathrm{QoC}$ in their wards. Team climate was the only work environment characteristic consistently associated with staff-reported QoC and seems an important factor to consider when improving QoC in nursing homes. Team climate refers to aspects like feeling safe, understood and supported, and having a shared vision.

As BRNs were expected to serve as informal leaders with the ability to lead improvements and redesign practice environments in nursing homes, particular attention was paid to their competencies, tasks and employment. Thirty-one experts from different countries reached consensus on 16 desirable competencies, which should, in the future, distinguish BRNs from other nursing staff working in nursing homes. As half of the competencies were related to leadership and coaching, competencies other than those traditionally associated with the nurse expert role are considered important. In our qualitative study, we found that having a vision on how to implement the BRN role within the nursing home is a key factor on whether or not an 
organization employs BRNs in their nursing homes. Within and between the organizations that employed BRNs, there was a large variation in the positioning, roles, tasks and responsibilities of BRNs. Difficulties that BRNs experienced when they started working in the nursing home were related to their role clarity, the naming of the BRN role, the extent to which BRNs received support, the openness of the direct care teams, and the interactions of the BRNs with other staff members. The added value direct care staff members experienced of BRNs differed among organizations.

\section{METHODOLOGICAL CONSIDERATIONS}

While specific methodological considerations regarding each study were described in the previous chapters, some general strengths and weaknesses of the study methods will be addressed further, i.e., the study design, the measurement of staffing, and the measurement of QoC.

\section{Study design}

To examine the relationship between direct nursing care staffing and $\mathrm{QoC}$ in nursing homes, two different observational study methods were conducted. First, a systematic review of longitudinal studies (Chapter 2), and second, cross-sectional studies, using data from the LPZ-database ("Landelijke Prevalentiemeting Zorgproblemen"), and from interviews and questionnaires (Chapters 3-5). Summarizing evidence from or conducting randomized controlled trials on staffing and $\mathrm{QoC}$ would have been most desirable to assess causal effects, but conducting and designing these studies in this domain is expensive, difficult and therefore only feasible on a small-scale. ${ }^{1}$ There are studies that approached a quasi-experimental design in situations where staffing levels were increased systematically on a large scale, ${ }^{1}$ for example, after policy changes in minimum staffing standards in the United States. ${ }^{2}$ Nevertheless, in most reported studies on staffing and QoC, observational designs are used. ${ }^{3}$ Although longitudinal observational designs are preferred, some are stronger than others. ${ }^{35}$ In our systematic review, we found that reported studies often use a panel-design, measuring staffing and QoC at different points in time. ${ }^{1,4}$ These panel-designs might not be better than crosssectional designs, as study participants might be different at each data collection point. ${ }^{5}$ In addition, we saw in our systematic review that the timing of measuring staffing characteristics was not described clearly in every included study. ${ }^{4}$

Ideally, studies should be able to demonstrate that changes in staffing levels precede changes in QoC outcomes. Nevertheless, even if this is the case, there still is a potential for bias, as changes in staffing levels might be a result of changes in resident characteristics (e.g., residents with more comorbidities), which preceded changes in QoC outcomes, too. ${ }^{6}$ In our study we used the LPZ-database. Although the LPZ is 
conducted twice a year, most organizations only participate once a year. In our case, conducting a longitudinal study based on the LPZ would have resulted in a panel-study, collecting data at two points in time, with a time lag of one year. We considered the possibility to conduct a prospective, longitudinal cohort study, comparing two subcohorts, i.e., nursing homes with higher and lower (BRN) staffing levels. However, in our cross-sectional studies, we saw little variance in staffing levels. For example, there were very few nursing homes with high BRN staffing levels. Moreover, too few (B)RNs were working in the participating wards to examine the relationship between professional staff mix (\% registered nurses/total staff) with QoC. We therefore concluded that conducting large-scale, longitudinal studies would not be appropriate. As little was known on the relationship between nurse staffing and $\mathrm{QoC}$ in Dutch nursing homes, conducting cross-sectional studies in collaboration with the LPZ was considered a relevant starting point to explore the relationship for the Dutch setting.

\section{Measurement of staffing}

In this dissertation, staffing data were collected at the ward level. This can be considered a strength, as many studies in this field examine staffing levels at facility level only. ${ }^{4}$ Considering staffing at the ward level enabled us to distinguish between somatic and psychogeriatric wards, while facility level analyses presume that the effect of staffing is the same for each ward in the facility. ${ }^{6}$ Nevertheless, analyzing staffing at the ward level means that one presumes that a) each resident within a ward receives the same amount of care, and b) the effect of staffing is the same for each resident, which might not necessarily be the case. Ideally, staffing should be measured at the level of the individual resident. This is hardly feasible in large-scale studies, as accurate measurements on individual level would require direct observations of staff-resident encounters. In terms of Donabedian's QoC framework, ${ }^{7}$ direct observations would provide more insight into process aspects of staffing (e.g., roles, tasks, how staff is scheduled, consistency of staff (same staff members taking care for same group of residents)), while in our large-scale study, only structural aspects of the concept were considered. ${ }^{1}$ Nevertheless, even when measuring staffing at individual level, it is hard to control for factors that may compensate lower staffing levels, like physician or allied professionals staffing levels, ${ }^{1}$ labor-saving technologies (e.g., digitalization of administrative processes, nurse call system, camera supervision), ${ }^{1,8,9}$ or the physical environment of the nursing home (e.g., length of hallways, location of medications and other equipment). ${ }^{35}$

\section{Measurement of QoC}

Two different methods were used to measure QoC in nursing homes. First, clinical outcomes that were measured in the LPZ (i.e., nosocomial pressure ulcers, medication 
incidents, falls, antipsychotic drug use, indwelling urinary catheter use) were considered (Chapters 3 and 4). Second, in a subsample of nursing home wards, staff-perceived QoC was also assessed (Chapters 4 and 5).

Clinical QoC outcomes were the most likely to be considered when assessing QoC in nursing homes. ${ }^{10}$ This was also found in our systematic review. ${ }^{4}$ More than a decade ago, various authors claimed that since QoC in nursing homes is a multi-dimensional construct, then developing a comprehensive measure reflecting the most important dimensions of QoC would be necessary. ${ }^{11-14}$ According to Donabedian, QoC consists of at least two interrelated dimensions, i.e., technical care (related to the management of health problems) and interpersonal processes (the psychosocial interaction between resident and staff member). ${ }^{7,15}$ With regard to technical care, nurse sensitive clinical outcomes have been studied widely to assess QoC in nursing homes. ${ }^{4}$ Interpersonal processes can be assessed by integrating resident, family and staff perspectives when assessing QoC in nursing homes, however, this is seldom done. ${ }^{16}$ To obtain insight into both dimensions of QoC in our studies, we combined clinical QoC outcomes with staffperceived QoC.

When assessing QoC, staff members may tend to consider QoC for all residents living in that ward. Ideally, we should have assessed resident and family perspectives, too, as they might provide insight into 'more subjective or interpersonal aspects of care $^{\prime}{ }^{17}$ and are more likely to only consider their personal experiences.

\section{THEORETICAL CONSIDERATIONS}

Our findings demonstrate that the prevalence of clinical QoC problems in the participating Dutch nursing home wards was low. Direct nursing care staff, in general, gave high mean ratings for QoC (overall QoC grade 7.7 in psychogeriatric wards and 7.4 in somatic wards on scale ranging from 1-10). They also agreed, to a great extent, with the statement 'In the event that a family member had to be admitted to a nursing home now, I would recommend this ward'. These findings suggest that there is a discrepancy between the negative public image of nursing homes and QoC as assessed in our studies.

As QoC is a multidimensional construct, ${ }^{7}$ our focus on clinical and staff-perceived QoC outcomes only accounts for some of the care that is provided. ${ }^{18,19}$ Obtaining insight into residents and family members' experiences may contribute to a more detailed understanding of $\mathrm{QoC}$. Therefore, to better acknowledge the multidimensionality of the concept, resident and family perspectives should also be considered in QoC assessments. Nevertheless, it might be impossible to encompass all dimensions of QoC. In addition, similar to outcomes, the structural and process aspects of QoC seem to be multidimensional, as our studies revealed that focusing on quantity of direct nursing care staff alone is inadequate to assess the relationship with QoC. Beyond numbers of 
staff, aspects that relate to the quality of staff should be considered. For example, staff members' skills and competencies, the roles they fulfill, or characteristics of a team's work environment.

Our results suggest that team climate may be an important work environment characteristic to consider when assessing QoC. When staff members have the feeling that the team climate is safe, they are more likely to openly address issues that could be improved. ${ }^{20}$ This may enable team-learning, e.g. by asking for help, discussing problems or learning from errors. ${ }^{21}$ Teams with increased learning behavior are expected to be more successful in dealing with innovations and change, ${ }^{20}$ as team members have learned to address their wishes and needs. ${ }^{21}$ In daily practice, team members might challenge the status quo more often, as they have learned to reflect on organizational issues that could be improved. ${ }^{21}$ Teams that are able to generate new ideas and working methods are, for example, better able to respond to changes in resident preferences. Therefore, a positive team climate that increases a team's innovativeness might contribute to better $\mathrm{QoC}$ in nursing homes. Besides improving QoC, a better team climate may also lead to an improvement of other work environment characteristics (e.g., better communication and coordination), which might also contribute to QoC. Thus, team climate may mediate the relationship between other work environment characteristics and QoC, and should be considered as a potential mediator.

Besides team climate, we found that clinical leaders who act as coaches might be an important aspect to consider when trying to improve QoC in nursing homes. Clinical nurse leaders are expected to be able to improve QoC at the bedside, without fulfilling a formal leadership position (e.g., ward/location manager). ${ }^{22-24}$ Stanley ${ }^{25}$ defines a clinical nurse leader as 'a clinician who is an expert in their field, and who, because they are approachable and effective communicators, are able to act as a role model. In this role they are empowered to motivate others to align their values and beliefs about nursing care to their daily practice. However, clinical nurse leaders' influence on QoC is primarily studied theoretically and should be explored further. Theoretically, clinical nurse leaders are expected to facilitate effective communication, strengthen intra- and interprofessional relationships, build and sustain teams that strive for a common goal, and provide daily support to direct nursing care staff. ${ }^{26}$ As they are present in the ward, they are considered to give feedback in real-time, to continuously monitor care delivery in a non-threatening way and to promote and sustain the uptake of evidence based practices through role modelling. ${ }^{26}$

In two of our studies (Chapters 6 and 7), the clinical nurse leader role in nursing homes was considered important and BRNs were expected to fulfill this role. Contrary to these expectations, one of our studies (Chapter 3) showed that in $43 \%$ of the 282 participating wards, no BRN was employed. In addition, in wards that employed a BRN, the amount of time spent on indirect care practices (e.g. staff education, coaching and care innovation projects) was low. This indicates that only few clinical nurse leaders are working in Dutch nursing homes. In other countries, master-educated RNs that work in 
direct resident care fulfill this clinical nurse leader role. ${ }^{26}$ However, in Dutch nursing homes, master-educated RNs often work as physician extenders. At the same time, based on what is theoretically expected from clinical nurse leaders, it is questionable whether or not BRNs are able to fulfill this role. For example, international experts in one of our studies (Chapter 6) stressed that a successful implementation of innovations in care practice may finally depend on the collaboration between master-educated RNs and BRNs. Experts saw master-educated RNs as those professionals providing evidence on best practice and ensuring evidence-informed care delivery, while BRNs were expected to oversee the implementation of best practice guidelines at an operational level. To conclude, our findings suggest that a reconsideration of the roles of mastereducated RNs and BRNs in Dutch nursing homes deserves attention.

\section{FUTURE DIRECTIONS}

The results of this dissertation have several implications for practice, education, policy and research.

\section{Practice}

Our findings showed that in 91\% of the wards that employed a BRN, BRNs spent at least part of their time on direct care. When working in direct care, their responsibilities may not differ from those of other direct nursing care staff members. If so, organizations may not be optimally utilizing them. To ensure that BRNs are employed to their full scope of practice, and lower-educated staff members are not going beyond their practice scope, ${ }^{27}$ the role of each staff member (e.g., BRN, RN, CNA) working in direct resident care should be differentiated. Nursing home organizations should develop a vision on the employment of each staff member to ensure that their goals, responsibilities and added value become clear for everyone in the organization, while assuring that they work together as a team. The development of such a vision requires buy-in at the board level.

Second, our findings suggest that nursing home organizations should assure that the team climate of direct nursing care teams is positive and safe. When staff members have the feeling that the team climate is safe, they are more likely to talk openly about and learn to reflect on issues that could be improved. ${ }^{20}$ A positive team climate might lead to staff members who challenge the status quo more often. ${ }^{21}$ Ideally, direct nursing care staff members should also feel safe when collaborating with other health professionals (e.g., nursing home medical specialists, psychologists, physical therapists) that are employed by the nursing home.

Third, findings of this dissertation indicate that informal clinical leaders who act as role models play an important role in nursing homes. Organizations should invest in 
clinical nurse leaders in direct resident care, as they might assist managers in creating a positive work environment for direct nursing care teams. ${ }^{23}$ Our findings suggest that BRNs, as the highest educated direct care staff members working in Dutch nursing homes, can likely fulfill this role. At the same time, our studies demonstrated that different types of role models exist. Therefore, nursing homes should determine which nurses are viewed by staff as role models, as they might have an influence (positive or negative) on a team's performance. Role models are not identified easily, and might therefore be an underutilized asset for the improvement of nursing home quality. ${ }^{23}$

\section{Education}

Our findings indicate that the amount of BRNs working in Dutch nursing homes is low. In our qualitative study (Chapter 7), directors and managers of organizations that do not actively employ BRNs within their nursing homes experience difficulties in attracting and retaining BRNs. BRN students complete internships in nursing homes, however, many students continue to stigmatize these as an undesirable work setting. ${ }^{28}$ One problem is that often, nursing homes are the first clinical setting where BRN students do their internships. ${ }^{29}$ These early placements mean that BRN students practice basic nursing skills (e.g., personal hygiene or bed baths) and thus view the experience in the nursing home as a setting that is not complex. ${ }^{27}$ These basic nursing skills can be acquired in another setting. Universities of applied sciences should also assign nursing home placements for BRN students in their third or fourth year of training, thus enabling students to obtain insight into the complexity of working in nursing homes. Such placements will facilitate BRN students to rethink the nursing home as a potential work setting. ${ }^{27}$

Second, internships should enable BRN students to gain meaningful experiences. As not all nursing homes employ BRNs, it is likely that BRN students are supervised by nonBRN staff working in nursing homes. Another challenge is that the understanding regarding the scope of the internship might be less clear for these organizations. ${ }^{28}$ Due to a lack of role models, organizations might not be able to position interns in a way that they receive meaningful experiences. As a consequence, the nursing home will remain an undesirable setting to work in. Educators should form local partnerships with nursing home organizations to assure that BRN students witness the complex work of BRNs in nursing homes and can practice at an expanded level. ${ }^{28}$ This will lead to an improvement of internships. Besides preparing BRN students for working with older adults, internships in nursing homes should clarify the role of BRNs in this setting, enticing graduates to work in nursing homes. ${ }^{28}$

Third, educational programs should equip graduates with skills and competencies that are considered necessary for working in future nursing homes. Even though it is impossible to forecast the future, it is for example expected that nursing homes will play an important role in providing complex psychogeriatric care, geriatric 
rehabilitation, and palliative care. Moreover, the role of technology is expected to increase. Specifically for BRN students, leadership and coaching competencies are considered important. The Dutch 'Bachelor Nursing 2020' profile ${ }^{30}$ was developed with the aim to equip BRN graduates with skills and competencies that meet future demands. Nevertheless, revisions of other curricula (e.g., CNAs) seem desirable, too.

\section{Policy}

As no consistent evidence for a positive relationship between direct nursing care staffing and QoC in nursing homes was found, the evidence base for implementing a generic minimum staffing standard for Dutch nursing homes is lacking. Still, probably there is a minimum staffing threshold below which the probability of poor QoC outcomes is higher. Instead of focusing on the quantity of staff, we welcome recent initiatives that instead consider team quality. ${ }^{31}$ At the same time, it might be desirable to explore further how direct nursing care staff working in Dutch nursing homes experiences staffing in their ward. A perceived lack of adequate staffing might lead to implicit rationing of care (also referred to as 'care left undone', 'missed care' or 'omitted care'), especially omitting social aspects of care delivery. ${ }^{32}$ Focusing on the completion of tasks (e.g., breakfast should be completed at a posted time, baths are given according to schedules) might result in (unnecessary) stress, ${ }^{33}$ leading to a feeling that staffing is not adequate. In this situation, reconsideration of work routines might change staffs' perception on staffing.

Second, due to a lack of scientific evidence, it is difficult to convince nursing home organizations that rethinking their current staff allocation (e.g., employing bettereducated staff) might improve QoC. The government should encourage local initiatives in which nursing home organizations experiment with a new mix of staff members or with more differentiated staff roles. Although the way in which these organizations have allocated their staff should not be employed as a blue-print by others, their 'lessons learned' could inspire and help other organizations to reconsider their staff allocation, too.

Third, future nursing home residents will have more influence on how their care is organized. In practice, this may mean that a balance has to be found between fulfilling resident needs and delivering care that is adequate and safe. Moreover, there might be discrepancies between preferences of residents themselves and what family members find important. Ideally when residents make choices regarding the organization of their care, they should be guided by staff that are competent to assess whether care delivery is adequate and safe, and are able, when needed, to mediate between residents and family members. It is likely that the current nursing home workforce is not prepared to guide residents in this process. Therefore, a careful preparation of the workforce (e.g., by training programs or coaching on the job) is considered desirable. 


\section{Research}

Methodological work is needed to improve measurement of resident perspectives of QoC. As resident preferences can change quickly, instruments that can provide insight into real-time preferences are desirable. Ideally, to make them feasible for improving daily QoC, these instruments should be user-friendly for direct nursing care staff members. At the same time, instruments should not be too burdensome for residents. Therefore, a careful evaluation of the appropriate frequency of various measures is recommended.

Second, conducting large-scale studies on the relationship between BRN staffing levels and $\mathrm{QOC}$ in Dutch nursing homes may not provide new insights since probably BRN staffing levels still are too low to adequately examine this relationship. Instead of studying staffing levels, more insight should be obtained into what BRNs are actually doing in nursing homes. Conducting direct observations may lead to a better understanding of how BRNs work together with direct nursing care staff members or how they contribute to QoC in nursing homes.

Third, our proposed theoretical model should be further refined in future longitudinal studies. These studies should consider work environment characteristics as potential mediators. More information on specific work environment characteristics (e.g., team climate) that contribute to QoC in nursing homes would provide an evidence base for the development of interventions aimed at improving these specific characteristics.

Fourth, while informal leaders who serve as role models for other staff members are considered a 'hidden treasure ${ }^{23}$ in healthcare in general and, due to the high amount of low-educated staff, in nursing homes in particular, a common understanding of and evidence for the role are lacking. ${ }^{22}$ Therefore, more insight should be obtained into characteristics of informal clinical leaders, their leadership behavior and the influence they have on team performance and QoC. In addition, it should be assessed whether or not BRNs are the professionals who should ideally fulfill this role in nursing homes. 


\section{REFERENCES}

1. Arling G, Mueller C. Nurse staffing and quality: the unanswered question. I Am Med Dir Assoc. 2014;15:376-378.

2. Lin $H$. Revisiting the relationship between nurse staffing and quality of care in nursing homes: an instrumental variables approach. J Health Econ. 2014;37:13-24.

3. Welton JM. Nurse staffing and patient outcomes: Are we asking the right research question? Int J Nurs Stud. 2016;63:A1-A2.

4. Backhaus R, Verbeek H, Van Rossum E, Capezuti E, Hamers JPH. Nurse staffing impact on quality of care in nursing Homes: a systematic review of longitudinal studies. J Am Med Dir Assoc. 2014;15:383-393.

5. Caruana EJ, Roman M, Hernández-Sánchez J, Solli P. Longitudinal studies. J Thoracic Dis. 2015;7:E537E540.

6. Griffiths P, Ball J, Drennan J, et al. Nurse staffing and patient outcomes: strengths and limitations of the evidence to inform policy and practice. A review and discussion paper based on evidence reviewed for the National Institute for Health and Care Excellence safe staffing guideline development. Int J Nurs Stud. 2016;63:213-225.

7. Donabedian A. The definition of quality and approaches to its assessment. Ann Arbor, MI: Health Administration Press; 1980.

8. Zúñiga F. The significance of staffing and work environment for quality of care and the recruitment and retention of care workers. Perspectives from the Swiss Nursing Homes Human Resources Project (SHURP). Basel: University of Basel; 2015.

9. Thoma-Lürken T, Bleijlevens MHC, Lexis MAS, Hamers JPH, De Witte LP. An overview of potential laborsaving and quality-improving innovations in long-term care for older people. J Am Med Dir Assoc. 2015;16:482-489.

10. Spilsbury K, Hewitt C, Stirk L, Bowman C. The relationship between nurse staffing and quality of care in nursing homes: A systematic review. Int J Nurs Stud. 2011;48:732-750.

11. Arling G, Kane R, Lewis T, Mueller C. Future development of nursing home quality indicators. Gerontologist. 2005;45:147-156.

12. Mor V, Angelelli J, Jones R, Roy J, Moore T, Morris J. Inter-rater reliability of nursing home quality indicators in the US. BMC Health Serv Res. 2003;3:1.

13. Mor V, Berg K, Angelelli J, Gifford, Morris J, Moore T. The quality of quality measurement in US nursing homes. Gerontologist. 2003;43:37-46.

14. Sangl J, Saliba D, Gifford D, Hittle D. Challenges in measuring nursing home and home health quality: Lessons from the first national healthcare quality report. Med Care. 2005;43:124-132.

15. Nakrem S, Vinsnes AG, Seim A. Residents' experiences of interpersonal factors in nursing home care: a qualitative study. Int J Nurs Stud. 2011;48:1357-1366.

16. Nakrem S, Vinsnes AG, Harkless GE, Paulsen B, Seim A. Nursing sensitive quality indicators for nursing home care: international review of literature, policy and practice. Int J Nurs Stud. 2009;46:848-857.

17. Li Y, Li Q, Tang Y. Associations between family ratings on experience with care and clinical quality-of-care measures for nursing home residents. Med Care Res Rev. 2016;73:62-84.

18. Dy SM, Herr K, Bernacki RE, Kamal AH, Walling AM, Ersek M, Norton SA. Methodological research priorities in palliative care and hospice quality measurement. J Pain Symptom Manage. 2016;51:155162.

19. Richards DA. Complex interventions and the amalgamation of marginal gains: A way forward for understanding and researching essential nursing care? Int J Nursing Stud. 2015;52:1143-1145.

20. Edmondson AC, Lei Z. Psychological safety: The history, renaissance, and future of an interpersonal construct. Annu Rev Organ Psychol Organ Behav. 2014;1:23-43. 


\section{GENERAL DISCUSSION}

21. Leicher $\mathrm{V}$, Mulder $\mathrm{RH}$. Team learning, team performance and safe team climate in elder care nursing. Team Performance Management: An International Journal. 2016;22:399-414.

22. Chávez EC, Yoder LH, editors. Staff nurse clinical leadership: a concept analysis. Nurs Forum. 2015;50:90100.

23. Downey M, Parslow S, Smart M. The hidden treasure in nursing leadership: Informal leaders. J Nurs Manag. 2011;19:517-521.

24. Lee $H$, Cummings GG. Factors influencing job satisfaction of front line nurse managers: a systematic review. J Nurs Manag. 2008;16:768-783.

25. Stanley D. Recognizing and defining clinical nurse leaders. Br J Nurs. 2006;15:108-118.

26. Bender M. Conceptualizing clinical nurse leader practice: an interpretive synthesis. J Nurs Manag. 2016;24:E23-E31.

27. McGilton KS, Bowers BJ, Heath $\mathrm{H}$, et al. Recommendations from the international consortium on professional nursing practice in long-term care homes. J Am Med Dir Assoc. 2016;17:99-103.

28. Nolet K, Roberts T, Gilmore-Bykovskyi A, et al. Preparing tomorrow's nursing home nurses: the Wisconsin long term care clinical scholars program. Gerontol Geriatr Educ. 2015;36:396-415.

29. Carlson E, Idvall E. Nursing students' experiences of the clinical learning environment in nursing homes: a questionnaire study using the CLES+ T evaluation scale. Nurse Educ Today. 2014;34:1130-1134.

30. Landelijk Overleg Opleidingen Verpleegkunde. Bachelor Nursing 2020: Een toekomstbestendig opleidingsprofiel 4.0. Stuurgroep Bachelor of Nursing 2020; 2015.

31. Zorginstituut Nederland. Kwaliteit verpleegzorg. https://www.zorginstituutnederland.nl/kwaliteit/kwaliteit+zorg+verpleeghuizen. Accessed November 7, 2016.

32. Zúñiga F, Ausserhofer D, Hamers JPH, Engberg S, Simon M, Schwendimann R. The relationship of staffing and work environment with implicit rationing of nursing care in Swiss nursing homes - A cross-sectional study. Int J Nurs Stud. 2015;52:1463-1474.

33. Syed I, Daly T, Armstrong P, Lowndes R, Chadoin M, Naidoo V. How do work hierarchies and strict divisions of labour impact care workers' experiences of health and safety? Case studies of long term care in Toronto. Jour Nursing Home Res. 2016;2:41-49 

Summary 
The aim of this dissertation is to examine direct nursing care staffing in nursing homes. Besides considering staffing levels, particular attention is paid to the competencies, tasks and employment of baccalaureate-educated registered nurses (BRNs), as these are expected to serve as informal leaders that have the ability to lead improvements and redesign practice environments in nursing homes. More specifically, this dissertation provides insight into the relationship between direct nursing care staffing, staff-related work environment characteristics and quality of care (QoC) in nursing homes. In addition, with a focus on BRNs, we report on future desirable distinguishing competencies of BRNs and how BRNs can be employed in nursing homes in ways that encourage their ability to add value to QoC.

Chapter 1 introduces the central concepts of this dissertation, i.e., 'nursing homes', 'direct nursing care staff' and 'quality of care', and presents the main objectives of this dissertation.

The results of a systematic review of 20 longitudinal studies examining the relationship between nurse staffing and $\mathrm{QoC}$ in nursing homes are presented in Chapter 2. No consistent evidence was found for a positive relationship between nurse staffing and QoC. Higher staffing levels were associated with better as well as lower QoC indicators. For example, for restraint use both positive (ie, less restraint use) and negative outcomes (ie, more restraint use) were found. With regard to pressure ulcers, we found that more staff led to fewer pressure ulcers and, therefore, better results, no matter who (registered nurse, licensed practical nurse/ licensed vocational nurse, or nurse assistant) delivered care. Furthermore, major methodological weaknesses (e.g., timing of data collection, accuracy of staffing data) limited the interpretation of the results.

Chapter 3 reports on a cross-sectional study on the relationship between the presence of BRNs and clinical QoC conducted among 282 wards and 6,145 residents from 95 Dutch long-term care facilities. 57\% of the included wards employed at least one BRN. In these wards, the mean amount of time BRNs spent per resident was low (4.8 minutes per resident per day). BRNs conducted direct care practices (personal and nursing care, e.g. help with activities of daily living) on $91 \%$ of the wards that employed a BRN, and indirect care practices (e.g. staff education, coaching, and care innovation projects) on $80 \%$ of these wards. We found a considerable variation in prevalence rates among residents between somatic (more likely to have a nosocomial pressure ulcer, medication incident or indwelling urinary catheter) and psychogeriatric wards (more likely to fall or use antipsychotic drugs). For both ward types, no consistent evidence was found for a relationship between the presence of BRNs and QoC, controlling for background characteristics. Among residents living in somatic wards that employed BRNs, the probability of experiencing a fall and receiving antipsychotic drugs was higher, whereas the probability of having an indwelling urinary catheter was lower. Among residents living in psychogeriatric wards that employed BRNs, the probability of experiencing a medication incident was lower. For residents from both ward types, the 
probability of suffering from nosocomial pressure ulcers did not significantly differ for residents in wards employing BRNs.

We then cross-sectionally assessed the relationship between the number of total staff hours per resident per day (HPRD) and QoC in 55 Dutch nursing home wards (Chapter 4). Besides clinical QoC, staff-reported QoC was assessed in this study. Staff members graded the overall QoC on their ward (grade 1-10, higher score indicating better QoC) and were asked to which extent they agreed with the statement "In case a family member had to be admitted to a nursing home now, I would recommend this ward". For residents from psychogeriatric and somatic wards, the probability of experiencing nosocomial pressure ulcers, falls, antipsychotic drug use, or urinary indwelling catheters was not significantly associated with HPRD. For residents living in psychogeriatric wards, however, higher HPRD were associated with a higher probability of experiencing a medication incident. This was not demonstrated for residents living in somatic wards. In addition, a relationship between HPRD and staff-reported QoC for both ward types was not found, as HPRD were not significantly associated with staffreported QoC (grade for overall QoC; recommending the ward). These findings underscore that focusing on quantity of care might not improve QoC in nursing homes.

Chapter 5 presents a cross-sectional study examining the relationship between direct care staffing levels (measured as HPRD), work environment characteristics and staff-perceived QoC in 55 Dutch nursing home wards. Overall, staff members were satisfied with the QoC in their wards. Staff members from psychogeriatric wards scored higher on ward recommendation. A better team climate was related to better perceived QoC in both ward types. In somatic wards, there was a positive association between multidisciplinary collaboration and agreement by staff of ward recommendation for a family member. In psychogeriatric wards, a lower score on market culture, better communication/coordination and a higher rating for multidisciplinary collaboration were significantly associated with a higher grade for overall QoC. Total direct nursing care staffing, adhocracy culture, hierarchy culture, as well as role model availability were not significantly related to QoC. Although our findings propose that team climate may be an important factor to consider when trying to improve QoC, more evidence on which work environment characteristics lead to better QoC in nursing homes is needed.

Besides considering staffing levels, this dissertation contributes to the development of BRN roles in nursing homes by providing insight into their competencies, tasks and employment in nursing homes. The aim of the study reported in Chapter 6 was to reach consensus on competencies, which should in the future, distinguish BRNs from other nursing staff (e.g., vocationally trained registered nurses, certified nurse assistants) in nursing homes. In this study, thirty-one experts from different countries reached consensus on 16 desirable competencies for BRNs working in nursing homes. Half of the competencies were related to leadership and coaching, two to communication, three to evidence-based practice and three to client assessment and geriatric expertise. Some of the competencies are not traditionally associated with nursing expertise e.g. being a 
team leader, role model and coach within the nursing team. Therefore, although there is continuing uncertainty about the future of nursing homes, our findings indicate that revision of current nursing curricula, nurse training programs and nursing home job profiles might be needed to meet the medically and psychologically complex needs of nursing home residents.

Chapter 7 contains a qualitative study, aimed at obtaining insight into how organizations employ BRNs in nursing homes and what is the perceived added value in care practices that organizations experience from their employment. This study found that having a vision on how to utilize the BRN role within the nursing home is a key factor on whether or not an organization employs BRNs in their nursing homes. Organizations that do not employ BRNs expect that BRNs do not want to work in nursing homes while organizations that have a clear vision on how to use their role are successful in employment and retention of BRNs. In organizations that employ BRNs, the percentage of board members and managers with an RN background was higher. Within and between the organizations that employ BRNs, there is a large variation in the positioning, roles, tasks and responsibilities of BRNs. Difficulties that BRNs experienced when they started working in the nursing home were related to their role clarity, the naming of the BRN role, the extent to which BRNs received support, the openness of the direct care teams, and the behavior of BRNs. The added value direct care staff members experienced differed between organizations. Our findings suggest that BRNs might improve QoC in nursing homes and provide different implications for practice. A careful implementation and evaluation of the BRN role seems crucial for a successful employment.

The final chapter (Chapter 8 ) summarizes the main findings of this PhD project. In addition, it discusses methodological considerations related to the cross-sectional study design, the collection of staffing data at ward level, the conceptualization of direct nursing care staffing (i.e., HPRD, BRN present or not), and the measurement of QoC. Moreover, theoretical considerations related to 'good QoC' and staff-related factors (i.e., informal leadership, team climate) that might contribute to good QoC in nursing homes are discussed. Finally, the chapter presents future directions for practice, education, policy, and research. For example, it is recommended that nursing home organizations differentiate the role of each team member (e.g., BRN, certified nurse assistant) and invest in informal leaders and positive team climates. To ensure that BRN students gain meaningful experiences during their nursing home internships, educators and nursing home organizations should form local partnerships. Moreover, it is considered desirable that government encourages local initiatives in which nursing home organizations experiment with a new mix of staff members or with more differentiated staff roles. Finally, future studies should further explore how team climate and informal leadership can contribute to 'good $\mathrm{QoC}^{\prime}$ in nursing homes. 
Samenvatting 
De kwaliteit van zorg in verpleeghuizen staat volop ter discussie. Steeds vaker komen incidenten in de zorg in de media, waarna er vaak gepleit wordt voor 'meer handen aan het bed'. De aanname daarbij is dat meer personeel leidt tot betere kwaliteit van zorg. Echter, er is weinig wetenschappelijke kennis beschikbaar over de relatie tussen de personele bezetting en de kwaliteit van zorg in verpleeghuizen. Dit proefschrift geeft meer inzicht in deze relatie. Naast de personeelsomvang en het opleidingsniveau van personeel in het algemeen, wordt specifiek gekeken naar de competenties, taken en inzet van HBO-verpleegkundigen. Van HBO-verpleegkundigen wordt verwacht dat zij een voorbeeldfunctie op de werkvloer kunnen vervullen, die bijdraagt aan een verbetering van de kwaliteit van zorg.

Concreet is de relatie onderzocht tussen personele bezetting, werkomgevingsfactoren en kwaliteit van zorg in verpleeghuizen. Daarnaast zijn wenselijke competenties van HBO-verpleegkundigen in kaart gebracht, die hen in de toekomst zouden moeten onderscheiden van andere medewerkers in de directe zorg. Ook is gekeken naar hoe HBO-verpleegkundigen in verpleeghuizen ingezet zouden kunnen worden, wat hun gepercipieerde meerwaarde is en wat er nodig is om de functie van de $\mathrm{HBO}$-verpleegkundige in het verpleeghuis te implementeren.

Hoofdstuk 1 introduceert de centrale concepten van dit proefschrift, namelijk 'verpleeghuizen', 'medewerkers in de directe zorg' en 'kwaliteit van zorg'. Daarnaast worden de doelstellingen van dit proefschrift gepresenteerd.

De resultaten van een systematisch literatuuronderzoek naar longitudinale studies over de relatie tussen personele bezetting en kwaliteit van zorg in verpleeghuizen worden gepresenteerd in Hoofdstuk 2. Er werd geen eenduidig bewijs gevonden voor een positief verband tussen de personele bezetting en de kwaliteit van zorg. Een hogere personeelsbezetting ging gepaard met betere, maar ook met slechtere kwaliteitsuitkomsten. Voor vrijheidsbeperkende maatregelen werden bijvoorbeeld positieve (minder vrijheidsbeperking) en negatieve (meer vrijheidsbeperking) uitkomsten gevonden bij een grotere personeelsinzet. Met betrekking tot decubitus vonden we dat meer personeel leidde tot minder decubitus en daardoor betere kwaliteit zorg, onafhankelijk van wie (verpleegkundige, verzorgende of helpende) de zorg leverde. Methodologische tekortkomingen van de geïncludeerde studies (onder meer timing van meetmomenten, kwaliteit van data over personeelsbezetting) beperkten de interpretatie van de resultaten.

Hoofdstuk 3 beschrijft een cross-sectionele studie naar de relatie tussen de inzet van $\mathrm{HBO}$-verpleegkundigen op een afdeling en klinische indicatoren voor kwaliteit van zorg (bijvoorbeeld decubitus en valpartijen). De studie werd verricht op 282 verpleeghuisafdelingen met in totaal 6145 bewoners van 95 Nederlandse zorginstellingen. Op 57\% van de afdelingen werd minimaal één HBO-verpleegkundige ingezet. Als $\mathrm{HBO}$-verpleegkundigen werden ingezet, leverden deze gemiddeld iets minder dan 5 minuten zorg per bewoner per dag. Daarvan besteedden zij gemiddeld ongeveer vier minuten aan directe zorgverlening en één minuut aan innovatietaken 
(zoals scholing, coaching of zorgvernieuwingstrajecten). Op 20\% van de afdelingen die een HBO-verpleegkundige inzetten, werd deze niet ingezet voor innovatietaken.

Met betrekking tot de prevalentie van zorgproblemen vonden we een duidelijk verschil tussen bewoners van somatische (meer decubitus, medicijnincidenten en kathetergebruik) en psychogeriatrische afdelingen (meer valpartijen en antipsychoticamedicatie). Voor beide type afdelingen werd geen duidelijke relatie gevonden tussen het inzetten van HBO-verpleegkundigen en de kwaliteit van zorg (gecorrigeerd voor achtergrondkenmerken). Bewoners van somatische afdelingen met een $\mathrm{HBO}$-verpleegkundige hadden bijvoorbeeld een grotere kans op valpartijen en antipsychoticagebruik, en een kleinere kans op kathetergebruik, in vergelijking met bewoners van afdelingen die geen HBO-verpleegkundige inzetten. Bewoners van psychogeriatrische afdelingen met een $\mathrm{HBO}$-verpleegkundige hadden een kleinere kans op medicijnincidenten, in vergelijking met bewoners van afdelingen die geen HBOverpleegkundige inzetten. Voor bewoners van beide soorten afdelingen werd voor decubitus geen significant verschil gevonden tussen afdelingen die wel of niet HBOverpleegkundigen inzetten.

De resultaten van een cross-sectionele studie naar de relatie tussen de personeelsbezetting (totale hoeveelheid geleverde zorg) en de kwaliteit van zorg in 55 Nederlandse verpleeghuisafdelingen worden beschreven in Hoofdstuk 4. De personeelsbezetting hebben we in kaart gebracht middels teamleider interviews (teamleiders baseerden zich daarbij op het dienstrooster). De uren geleverde zorg op een afdeling hebben we afgezet tegen het aantal bewoners op een afdeling en zo een indicator voor de totale hoeveelheid geleverde zorg berekend. Naast klinische kwaliteitsindicatoren hebben we gekeken naar de subjectief ervaren kwaliteit door medewerkers. Medewerkers gaven bijvoorbeeld een rapportcijfer voor de kwaliteit op hun afdeling en gaven aan in hoeverre zij het eens waren met de stelling "Als een familielid nu moet worden opgenomen, zou ik deze afdeling aanbevelen." Voor bewoners van psychogeriatrische en somatische afdelingen werd geen significante relatie gevonden tussen de hoeveelheid geleverde zorg en de kans op decubitus, valpartijen, antipsychotica- of kathetergebruik. Voor bewoners van psychogeriatrische afdelingen was een grotere hoeveelheid zorg gerelateerd aan een grotere kans op medicijnincidenten. Voor beide soorten afdelingen werden geen significante relaties gevonden tussen de hoeveelheid zorg en de door medewerkers ervaren kwaliteit. Deze resultaten suggereren dat meer medewerkers op een afdeling niet per se zullen leiden tot een betere kwaliteit van zorg in verpleeghuizen.

Hoofdstuk 5 bespreekt een cross-sectionele studie naar de relatie tussen de totale hoeveelheid zorg (uren per bewoner per dag), werkomgevingskenmerken en de subjectief ervaren kwaliteit door medewerkers (rapportcijfer en stelling over aanbevelen afdeling). Deze studie werd verricht in 55 Nederlandse verpleeghuisafdelingen, die ook in hoofdstuk 4 centraal stonden. Over het algemeen waren medewerkers tevreden over de kwaliteit van zorg op hun afdeling. Medewerkers 
van psychogeriatrische afdelingen waren meer geneigd hun afdeling aan te bevelen. Voor beide type afdelingen (psychogeriatrie, somatiek) vonden we dat een positief teamklimaat een positief effect had op de kwaliteit van zorg. Voor psychogeriatrische afdelingen vonden we dat een minder marktgerichte cultuur (wat betekent dat de sfeer minder competitief is en het bereiken van concrete resultaten en output minder centraal staat), een betere communicatie/coördinatie en een betere multidisciplinaire samenwerking gerelateerd waren aan een hoger rapportcijfer voor de kwaliteit van zorg. Voor somatische afdelingen werd een positieve relatie gevonden tussen de multidisciplinaire samenwerking en het aanbevelen van de afdeling.

Naast informatie over de personeelsomvang en het opleidingsniveau van personeel in verpleeghuizen, beoogt dit proefschrift ook een bijdrage te leveren aan de discussie over de rol van HBO-verpleegkundigen in verpleeghuizen. Hierbij lag de focus op hun competenties, taken en inzet. Het doel van de studie, beschreven in Hoofdstuk 6, was het bereiken van consensus over competenties, die in de toekomst de HBOverpleegkundige zou moeten onderscheiden van andere medewerkers in de directe zorg (waar hun meerwaarde ten opzichte van de overige medewerkers zou kunnen liggen; bijvoorbeeld MBO-verpleegkundigen, verzorgenden). In deze studie hebben 31 experts uit verschillende landen 16 competenties geïdentificeerd. De helft van deze competenties had betrekking op leiderschap en coaching, twee competenties hadden betrekking op communicatie, drie op evidence-based werken en drie op diagnostiek en geriatrische expertise. Sommige van deze competenties zijn eerder niet zo expliciet gekoppeld aan de expertise van verpleegkundigen, bijvoorbeeld 'rolmodel en coach voor het team zijn'. Het is dan ook belangrijk om de huidige opleidings- en scholingsprogramma's, maar ook functieprofielen kritisch te blijven toetsen op hun toekomstbestendigheid.

Hoofdstuk 7 beschrijft een kwalitatieve studie naar verschillen in hoe men HBOverpleegkundigen in verpleeghuizen inzet, wat hun meerwaarde is en wat er nodig is om de functie van $\mathrm{HBO}$-verpleegkundige beter in het verpleeghuis te implementeren. In deze studie vonden we dat het hebben van een visie over hoe men HBOverpleegkundigen wil inzetten binnen het verpleeghuis bepaalt of organisaties hen daadwerkelijk inzetten. Organisaties die hen niet inzetten, verwachten dat HBOverpleegkundigen niet in een verpleeghuis willen werken. Organisaties met een duidelijke visie over de inzet van HBO-verpleegkundigen lukt het deze te werven en te binden. Tussen en binnen organisaties die HBO-verpleegkundigen inzetten waren grote verschillen te zien wat betreft hun positionering, rollen, taken en verantwoordelijkheden. Moeilijkheden waar HBO-verpleegkundigen in het begin van hun aanstelling tegenaan liepen, waren bijvoorbeeld gerelateerd aan rolonduidelijkheid, de mate van ervaren steun en de openheid van de directe zorgteams. Tussen organisaties zat er verschil in de door medewerkers (MBO-verpleegkundigen, verzorgenden, helpenden) ervaren meerwaarde van HBO-verpleegkundigen. Onze resultaten wijzen erop dat de inzet van $\mathrm{HBO}$-verpleegkundigen lijkt te kunnen bijdragen 
aan een betere kwaliteit van zorg zoals ervaren door zorgorganisaties. Een zorgvuldige implementatie en evaluatie van de rol van HBO-verpleegkundigen is cruciaal voor hun succesvolle inzet.

In het laatste hoofdstuk, Hoofdstuk 8, worden de belangrijkste bevindingen van dit proefschrift samengevat. Ook worden methodologische overwegingen met betrekking tot onder meer het cross-sectionele studie design en het meten van kwaliteit van zorg besproken. Daarna volgen theoretische overwegingen met betrekking tot 'goede kwaliteit van zorg' en medewerker-gerelateerde factoren (informeel leiderschap, teamklimaat) die een bijdrage lijken te kunnen leveren aan 'goede kwaliteit van zorg' in verpleeghuizen. Ten slotte worden aanbevelingen gedaan voor de praktijk, opleidingen, beleid en onderzoek. Een belangrijke implicatie is bijvoorbeeld om roldifferentiatie bij zorgmedewerkers te creëren en te investeren in informele leiders en een positief teamklimaat. Informele leiders en een positief teamklimaat kunnen bijdragen aan het lerend vermogen van een team, iets wat ook belangrijk wordt gevonden in het nieuwe kwaliteitskader verpleeghuiszorg. Om te waarborgen dat studenten $\mathrm{HBO}$-verpleegkunde tijdens hun stages in het verpleeghuis relevante en interessante ervaringen kunnen opdoen, worden opleidingen en verpleeghuisorganisaties aangeraden om intensievere lokale samenwerkingsverbanden te creëren. In toekomstig onderzoek moet onder andere bekeken worden hoe het teamklimaat en informeel leiderschap kunnen bijdragen aan 'goede kwaliteit van zorg' in verpleeghuizen. 

Zusammenfassung 
Ziel dieser Dissertation ist es, die personelle Besetzung in Pflegeheimen zu untersuchen. Neben dem Besetzungsgrad von Pflegepersonal in Pflegeheimen im Allgemeinen, fokussiert diese Dissertation auf die Kompetenzen, Aufgaben und den Einsatz von Bachelor-Pflegekräften. Von Bachelor-Pflegekräften wird erwartet, dass diese eine informelle Leitungsfunktion übernehmen, wodurch eine Pflegequalitätsverbesserung erreicht werden kann. Konkret bietet diese Dissertation Einblick in den Zusammenhang zwischen dem Besetzungsgrad, Arbeitsumgebungsfaktoren (z.B. Teamwork) und der Pflegequalität in Pflegeheimen. Des Weiteren, fokussierend auf Bachelor-Pflegekräfte, werden wünschenswerte Kompetenzen von Bachelor-Pflegekräften beschrieben, die diese zukünftig von anderen Pflegekräften in Pflegeheimen unterscheiden sollen. Auch wird beschrieben, wie Bachelor-Pflegekräfte in Pflegeheimen eingesetzt werden können, welchen Mehrwert sie haben und was nötig ist, um die Rolle der BachelorPflegekraft im Pflegeheim zu implementieren.

In Kapitel 1 werden die zentralen Konzepte dieser Dissertation beschrieben, nämlich ,Pflegeheime', ,Pflegeheimpersonal' und, Pflegequalität'. Im Anschluss folgt eine Beschreibung der Ziele dieser Dissertation.

Die Resultate einer systematischen Literaturstudie von 20 Längsschnittstudien, in denen der Zusammenhang zwischen dem Besetzungsgrad und der Pflegequalität in Pflegeheimen untersucht wurde, werden in Kapitel 2 präsentiert. Für einen positiven Zusammenhang zwischen dem Besetzungsgrad und der Pflegequalität wurde keine eindeutige Evidenz gefunden. Für Fixierung wurden zum Beispiel positive (weniger Fixation) und negative (mehr Fixation) Ergebnisse gefunden. Bezüglich Dekubitus fanden wir, dass mehr Pflegepersonal zu weniger Dekubitus führte, und demnach zu besseren Ergebnissen, unabhängig vom eingesetzten Pflegepersonal (examinierte Pflegekräfte oder Pflegehilfskräfte). Methodologische Schwierigkeiten (zum Beispiel bezüglich der Zeitpunkte der Messungen oder der Qualität der Daten zur personellen Besetzung) begrenzten die Interpretation der Resultate.

In Kapitel 3 wird eine Querschnittsstudie beschrieben, in welcher der Zusammenhang zwischen dem Einsatz von Bachelor-Pflegekräften und der Pflegequalität untersucht wurde. Diese Studie umfasste 282 Wohnbereiche und 6145 Bewohner von 95 niederländischen Einrichtungen der stationären Langzeitpflege. In $57 \%$ der Wohnbereiche wurde minimal eine Bachelor-Pflegekraft eingesetzt. In diesen Bereichen war die durchschnittliche Zeit, welche die Bachelor-Pflegekraft pro Bewohner im Wohnbereich verbrachte, niedrig (4,8 Minuten pro Bewohner pro Tag). Die BachelorPflegekräfte wurden in 91\% dieser Wohnbereiche in der direkten Pflege eingesetzt (persönliche und pflegerische Betreuung, zum Beispiel Hilfe bei Aktivitäten des täglichen Lebens) und in $80 \%$ dieser Wohnbereiche für indirekte Pflegeaktivitäten (zum Beispiel Schulung des übrigen Personals, Coaching, Pflegeinnovationsprojekte). Wir fanden eine beträchtliche Variation in den Prävalenzraten der Bewohner somatischer (mehr Dekubitus, Medikamentenfehler und Harnkatheter) und psychogeriatrischer Wohnbereiche (mehr Stürze und Antipsychotika). Für beide Wohnbereichstypen wurde 
keine eindeutige Evidenz für einen Zusammenhang zwischen dem Einsatz von BachelorPflegekräften und der Pflegequalität (kontrolliert für demografische Faktoren) gefunden. Unter Bewohnern somatischer Wohnbereiche auf denen eine BachelorPflegekraft eingesetzt wurde, war die Wahrscheinlichkeit zu Stürzen und Antipsychotika einzunehmen höher, während die Wahrscheinlichkeit einen Harnkatheter zu benutzen niedriger war (im Vergleich zu Wohnbereichen, die keine Bachelor-Pflegekraft einsetzen). Unter Bewohnern psychogeriatrischer Wohnbereiche auf denen eine Bachelor-Pflegekraft eingesetzt wurde, war die Wahrscheinlichkeit eines Medikamentenfehlers niedriger. Für Bewohner von beiden Wohnbereichstypen hatte der Einsatz einer Bachelor-Pflegekraft keinen Einfluss auf die Dekubitusrate.

In einer anderen Querschnittsstudie haben wir den Zusammenhang zwischen dem Personaleinsatz und der Pflegequalität in 55 Wohnbereichen niederländischer Pflegeheime untersucht (Kapitel 4). Den Personaleinsatz haben wir mittels Interviews (basierend auf dem Dienstplan) in Erfahrung gebracht. Um einen Vergleich mit anderen Wohnbereichen zu ermöglichen, haben wir die Arbeitsstunden, die pro Bereich pro Tag eingesetzt wurden, durch die Anzahl der Bewohner eines Bereiches geteilt. Neben klinischen Qualitätsindikatoren wurde in dieser Studie auch die Qualitätseinschätzung des Pflegepersonals berücksichtigt. Das Pflegepersonal beurteilte die allgemeine Pflegequalität des eigenen Wohnbereiches (Note 1-10, höhere Zahl bedeutet bessere Qualität) und wurde gefragt, in welchem Maße man der folgenden Aussage zustimmt: „Wenn ein Familienmitglied jetzt in einem Pflegeheim aufgenommen werden müsste, würde ich diesen Wohnbereich empfehlen". Für Bewohner beider Wohnbereichstypen (somatisch und psychogeriatrisch), wurde kein Zusammenhang zwischen dem Personaleinsatz pro Bewohner pro Tag und der klinischen Pflegequalität (Dekubitus, Stürze, Antipsychotika, Katheter) gefunden. Lediglich für Bewohner psychogeriatrischer Wohnbereiche fanden wir einen Zusammenhang zwischen einem höheren Personaleinsatz und einer höheren Häufigkeit von Medikamentenfehlern. Wir fanden keinen Zusammenhang zwischen den Beurteilungen der allgemeinen Pflegequalität durch das Pflegepersonal und dem Personaleinsatz.

Kapitel 5 präsentiert eine Querschnittsstudie, in welcher der Zusammenhang zwischen dem Personaleinsatz (pro Bewohner pro Tag), Arbeitsumgebungsfaktoren und der Qualitätseinschätzung durch das Pflegepersonal untersucht wurde. Diese Studie wurde in denselben 55 Bereichen niederländischer Pflegeheime durchgeführt. Im Allgemeinen war das Pflegepersonal zufrieden mit der Pflegequalität des eigenen Wohnbereiches. Pflegepersonal psychogeriatrischer Bereiche stimmte eher zu, den Wohnbereich weiter zu empfehlen. Ein besseres Teamklima hing mit einer höheren Qualitätseinschätzung zusammen. In somatischen Bereichen wurde ein positiver Zusammenhang zwischen der multidisziplinären Zusammenarbeit und dem Weiterempfehlen des Wohnbereiches gefunden. In psychogeriatrischen Bereichen hingen eine weniger auf den Markt gerichtete Kultur (weniger kompetitiv und weniger fokussiert auf konkrete Ziele und Resultate), eine bessere Kommunikation/Koordination 
und eine bessere multidisziplinäre Zusammenarbeit positiv mit der Benotung der Pflegequalität zusammen.

Neben Informationen zum Personaleinsatz und Ausbildungsniveau in Pflegeheimen, bezweckt diese Dissertation auch einen Beitrag an der Weiterentwicklung der Rolle der Bachelor-Pflegekraft im Pflegeheim zu leisten. Hierbei lag der Fokus auf den Kompetenzen, Aufgaben und dem Einsatz von Bachelor-Pflegekräften. Das Ziel der Studie, die in Kapitel 6 beschrieben steht, war es Konsens zu erreichen über die Kompetenzen, die in Zukunft Bachelor-Pflegekräfte vom anderen Pflegepersonal in Pflegeheimen unterscheiden sollten (möglicher Mehrwert von Bachelor-Pflegekräften im Vergleich zu anderen Mitarbeitern). In dieser Studie haben 31 Experten aus verschiedenen Ländern 16 Kompetenzen identifiziert. Die Hälfte der Kompetenzen gehörte zum Bereich 'Führung ('leadership') und Coaching', zwei Kompetenzen zu ,Kommunikation', drei zu ,Evidence-Based Practice' und drei zu ,Diagnostik und geriatrische Expertise'. Manche dieser Kompetenzen sind weniger explizit verknüpft an der Expertise von Pflegekräften, zum Beispiel ,Vorbild und Coach für das Team sein'. Darum ist es wichtig, bestehende Schulungs- und Trainingsprogramme, aber auch Jobprofile kritisch auf ihre Zukunftsbeständigkeit zu überprüfen.

Kapitel 7 beschreibt eine qualitative Studie zu verschiedenen Möglichkeiten des Einsatzes von Bachelor-Pflegekräften in Pflegeheimen, deren Mehrwert und was nötig ist, um die Rolle der Bachelor-Pflegekraft besser im Pflegeheim zu implementieren. In dieser Studie fanden wir heraus, dass das Haben einer Vision über den Einsatz von Bachelor-Pflegekräften darüber bestimmt, ob diese tatsächlich eingesetzt werden. In Organisationen, die diese nicht einsetzen, ging man davon aus, dass BachelorPflegekräfte nicht in Pflegeheimen arbeiten wollen. Organisationen mit deutlicher Vision über ihren Einsatz schafften es, diese zu werben und zu binden. Zwischen und innerhalb Organisationen, die Bachelor-Pflegekräfte einsetzen, sahen wir große Unterschiede bezüglich ihrer Positionierung, ihrer Rollen, ihrer Aufgaben und ihrer Verantwortungen. Schwierigkeiten womit Bachelor-Pflegekräfte zu Beginn ihrer Tätigkeit zu kämpfen hatten, hingen zum Beispiel mit der Undeutlichkeit ihrer Rolle, dem Maß erfahrener Unterstützung und der Offenheit von Pflegeteams zusammen. Zwischen den Organisationen gab es Unterschiede im durch das Pflegepersonal (examinierte Pflegekräfte ohne Bachelorabschluss, Pflegehilfskräfte) beschriebenen Mehrwert von Bachelor-Pflegekräften. Unsere Resultate weisen darauf hin, dass der Einsatz von Bachelor-Pflegekräften zu einer wahrgenommenen Qualitätsverbesserung führen kann. Eine sorgfältige Implementation und Evaluation der Rolle der BachelorPflegekraft scheint unabdingbar für ihren erfolgreichen Einsatz.

Im letzten Kapitel, Kapitel 8, werden die wichtigsten Resultate dieser Dissertation zusammengefasst. Auch werden methodologische und theoretische Gesichtspunkte dieser Dissertation diskutiert. Zuletzt werden Handlungsempfehlungen für Praxis, Lehre, Politik und zukünftige Forschung ausgesprochen. 
Valorization 
In this chapter, the societal value of this dissertation's findings will be discussed. These findings contribute to recent political and societal discussions on quality of care (QoC) in Dutch nursing homes.

Also, outside the Netherlands, it is a persistent belief that 'more hands' are needed to improve QoC in nursing homes. An increase in the number of staff is presumed to positively affect the QoC and quality of life of nursing home residents. Despite the heterogeneity across countries, nursing homes worldwide have to ensure the delivery of high QoC, and adequately staffing the homes remains a major concern in most countries. Direct nursing care staff vary in their educational level, thus it is a significant challenge to determine the numbers and types of staff as well as staffs' competencies that are necessary to meet the complex needs of nursing home residents. The findings of this dissertation have demonstrated that an increase in the number of staff will not, per se, lead to better QoC in nursing homes and have deepened the understanding of the influence of direct nursing care staff on QoC. To improve QoC in nursing homes, it seems necessary to think beyond numbers.

Findings of the studies described in Chapters 2-5 are published in a literature review that we conducted for the Dutch Ministry of Health, Welfare and Sport (VWS). VWS provided this report to delegates of the Dutch House of Representatives ('Tweede Kamer' in Dutch). It received a lot of media attention in July 2016 and has contributed to the recent quality framework for nursing homes ('Kwaliteitskader verpleeghuiszorg' in Dutch) of the National Health Care Institute ('Zorginstituut Nederland' in Dutch). This quality framework was published in January 2017 and provides temporary norms for among other quality aspects - nursing home staffing. Based on the results of our report, the National Health Care Institute concluded that the evidence base for a generic minimum staffing standard is lacking. Instead, more attention has to be paid to the quality of teams, which is in line with our findings.

The findings of this dissertation contribute to the further development of the quality framework's staffing norms, which are classified into three themes: (1) Attention, presence and surveillance, (2) Specific knowledge and skills, (3) Reflection, learning and developing. Based on our findings, we provide implications for each theme hereafter.

\section{Attention, presence and surveillance}

One norm related to 'attention, presence and surveillance' states that 'during intensive care moments (e.g., getting up, going to bed, intake and dying), at least two care providers are present to execute these tasks'. Basically, for direct nursing care staff, it is important to ask for and to get help in those moments that help is needed. Getting help if needed may increase the delivery of safe care. Therefore, it is important that care providers are able to critically reflect on what they do. Critical reflection allows them to estimate and indicate whether or not they need help. 
It is questionable whether it is possible to determine 'intensive care moments' in which help is needed. For example, in small-scale living groups, often one staff member works alone during a great extent of the day. When a resident falls, it might be necessary to ask a colleague - who is working in another living group within the facility - for help. In addition, based on the formulation of the norm, it does not become clear if the two care providers both need to be present in the ward or whether the second care provider can be called in if needed.

To further concretize this norm, the physical environment of a nursing home should be considered, too. In a stand-alone, small-scale living group with six residents, it might be financially impossible to guarantee that at least two care providers are present in the ward. One consequence may be that such small-scaled living groups disappear. Based on our findings, there is no reason to assume that two care providers (instead of one), per se, increase the QoC provided.

\section{Specific knowledge and skills}

One norm related to 'specific knowledge and skills' says '24/7, a BIG-registered registered nurse (RN; 'verpleegkundige' in Dutch) is present within 30 minutes.' We have to take care that this norm is not interpreted by nursing home organizations, as 'It is not necessary to employ RNs within direct care teams, as long as an RN can be called in.' The RN should not be seen as a professional who only executes the technical nursing tasks (e.g., placing a catheter). Due to the rising complexity, it is desirable to integrate (baccalaureate-educated) (B)RNs in direct resident care. Especially within a self-organizing team, it might be wise to employ (B)RNs who can support lowereducated staff members.

In the mentioned norm, no differentiation is made between vocationally trained RNs and BRNs. For nursing home organizations, it would be helpful to concretize desirable responsibilities of (B)RNs in nursing homes in the quality framework. Providing an overview of these responsibilities helps organizations to ensure that (B)RNs are employed to their full scope of practice and that the role of each staff member (e.g., $B R N, R N$, certified nurse assistant (CNA)) within an organization is differentiated. With regard to BRNs, we saw in one of our studies (Chapter 6 ) that competencies other than those traditionally associated with the nurse expert role are considered important, for example, competencies related to informal leadership and coaching. A BRN who is only present upon request may not be able to serve as informal leader and coach for a direct nursing care team.

Master-educated RNs are not mentioned in the quality framework's staffing norms. In Dutch nursing homes, these RNs nowadays often work as physician extenders. In the quality framework, a consideration of which role master-educated RNs should play in Dutch nursing homes deserves further attention. For example, international experts in one of our studies (Chapter 6 ) stressed that a successful implementation of innovations 
in care practice may finally depend on the collaboration between master-educated RNs and BRNs. They saw master-educated RNs as those professionals providing evidence on best practice and ensuring evidence-informed care delivery, while BRNs were expected to oversee the implementation of best-practice guidelines at an operational level. Therefore, it is wise to reconsider their mostly medically oriented positioning. Instead of taking over tasks from the nursing home medical specialist, they could, for example, play a role in the implementation and promotion of working methodologically. Since they have expertise in the fields of nursing and medicine, they might also be able to increase the interdisciplinary collaboration in nursing homes. Reconsidering the role of master-educated RNs can lead to a new career pathway for RNs in nursing homes, providing the opportunity to attract and employ more academically qualified staff in the future.

\section{Reflection, learning and developing}

In the future, working in nursing homes will become even more complex, not only due to an increase in residents' care needs but also due to the fact that nursing home residents and their informal network will have more influence on how their care is organized. Therefore, stimulating learning and development among the nursing home workforce (e.g., by training programs or coaching on the job) is considered desirable. One norm of the quality framework says 'For each care provider, there is enough time for learning and development via feedback, intervision, reflection and education. The amount and kind of [learning and development] are included in the quality plan.' Ideally, reflection, learning and developing should become a 'daily routine' integrated in the daily work of each direct nursing care team. To stimulate this, our results suggest that nursing home organizations should invest in positive team climates. When staff members have the feeling that the team climate is safe, they are more likely to openly address issues that should be improved. This may enable team learning, e.g., by asking for help, discussing problems or learning from errors. Besides team climate, we found that clinical leaders who act as coaches for the direct nursing care team might be an important aspect to consider. As they are present in the ward, they are considered to give feedback in real-time, to continuously monitor care delivery in a non-threatening way and to promote and sustain the uptake of evidence-based practices through role modeling.

Another norm related to 'reflection, learning and developing' says 'There is enough time available for primary responsible caregivers ('eerst verantwoordelijk verzorgenden' or 'contactverzorgenden' in Dutch) to participate in the multidisciplinary meeting ('MDO' in Dutch).' Giving primary responsible caregivers the opportunity to participate in the multidisciplinary meeting is desirable, as these caregivers are those employees that are expected to know the clients best. Nowadays, often CNAs fulfill this role. In our qualitative study (Chapter 7), we saw that one nursing home organization chose to 
position BRNs in a way that allowed them to closely collaborate with primary responsible caregivers and to coach them to fulfill their role. In another study (Chapter 6), international experts saw the BRN as the professional who is 'able to coordinate the multidisciplinary team' and is 'the professional who sees the whole picture, [who] should organize regular team meetings in which all disciplines participate; [and who] should manage the overall care coordination'. Therefore, it is questionable whether CNAs are able to fulfill the primary responsible caregiver role individually. Maybe they should fulfill this role in a tandem with BRNs. Alternatively, like in home care, it should be considered whether a BRN should fulfill the primary responsible caregiver role. A careful evaluation of which (combination of) caregiver(s) is able to meet the needs of residents is desirable.

\section{INNOVATIVE ASPECTS OF OUR STUDIES}

Outside the United States, research on the relationship between direct nursing care staffing and QoC in nursing homes is scarce. The study presented in Chapter 3 is the first large-scale study that provides insight into the amount and responsibilities of BRNs working in Dutch nursing care wards. Although different Dutch stakeholders have made efforts to increase the amount of BRNs in elderly care, the study shows that the amount of BRNs in institutional elderly care is still low.

In many countries, the amount of BRNs working in nursing homes is low; obtaining more information on how to best allocate them in nursing homes was considered desirable. To allocate them optimally, it is important to obtain insight into their competencies first. When studying existing competency profiles, we felt that these profiles did not provide sufficient clarity on which competencies distinguish BRNs from other direct nursing care staff. In addition, as worldwide significant changes are occurring in the nursing home sector, we were asking ourselves how future-oriented and setting-specific the existing profiles were. Contrary to other studies on the competencies of direct nursing care staff in elderly care, we did not include BRNs that are currently working in a nursing home in our panel, since they would likely be influenced by their current working conditions and thus would have trouble envisioning alternative views of staff allocation.

To blaze a trail for BRNs in nursing homes, we conducted an exploratory, qualitative study (Chapter 7) to obtain insight into factors that can contribute to the development of BRN roles in nursing homes. In addition, we wanted to explore why some organizations succeed in employing BRNs in nursing homes, while other organizations do not. This study showed, for example, that organizations that have a clear vision on how to employ BRNs within the nursing homes do not experience great recruiting and retention problems. Therefore, 'recruiting and retention problems' might no longer be an important argument for not employing BRNs in nursing homes. 


\section{DISSEMINATION OF FINDINGS}

Already early in the project, we started to disseminate our results. For the scientific audience, five out of the six studies presented in this dissertation are published in peerreviewed, international journals. Two of the articles are published 'open access', meaning that they are freely accessible for everyone interested in these studies. Another way to share our findings with other researchers and professionals was by giving (scientific) presentations at several national and international conferences. As the project was embedded within the Living Lab in Ageing \& Long-Term Care, findings have been spread early among the long-term care organizations that participate in the Living Lab, too, for example, by giving lay presentations for direct nursing care staff and managers of these organizations. During the whole project, findings were discussed with representatives from national stakeholder organizations (branche organization Actiz, client organization LOC, the Dutch Nurses Association (V\&VN), the Health Care Inspectorate (IGZ) and VWS). The report of the literature study conducted for VWS is freely accessible at:

https://www.rijksoverheid.nl/documenten/rapporten/2016/07/04/meer-is-niet-per-sebeter.

To disseminate the main findings of this dissertation further, several steps will be undertaken. Through writing accessible summaries, we hope to reach a wider audience. A Dutch summary as well as an e-book of this dissertation will be made available on the website of the Living Lab (http://www.academischewerkplaatsouderenzorg.nl). The ebook will also be made available via the platform 'Proefschriften Verpleegkunde' (http://www.proefschriftenverpleegkunde.nl). This platform aims to make scientific knowledge in the field of nursing more accessible for students, educators and nurses in the Netherlands and Flanders. As this dissertation revealed relevant insights into the role of baccalaureate-educated registered nurses (BRNs) in nursing homes, particular efforts will be taken to inform these nurses. To achieve this, we will be in contact with the network of Dutch BRNs specialized in gerontology and geriatrics (http://www.hbovgg.net) and the steering group of the Dutch campaign ' $\mathrm{HBO}-\mathrm{V}$ in de ouderenzorg - daar zit meer achter' (http://www.daarzitmeerachter.nl). To disseminate the findings internationally, an English and German summary will be made available, too.

Part of the results of this dissertation will be included in a handbook, which provides nursing home organizations with concrete guidance on how to reach a more optimal staff mix between BRNs and other staff members working in nursing homes. This handbook will be developed in 2017. 
Acknowledgements 
Het is af! Oder wie Giovanni Trapattoni sagen würde: „Ich habe fertig!“

Nu dan nog het meest gelezen hoofdstuk uit een proefschrift: het dankwoord. Om maar meteen met een cliché te beginnen: het is waar, promoveren doe je niet alleen!

Dit gezegd hebbend, wil ik graag iedereen bedanken die direct of indirect heeft bijgedragen aan de totstandkoming van mijn proefschrift. Zonder iemand te kort te willen doen, wil ik een paar mensen in het bijzonder noemen.

Allereerst gaat mijn dank uit naar iedereen die heeft deelgenomen aan mijn onderzoek. Naast de medewerkers van de deelnemende verpleeghuisorganisaties, dank ik ook de 'experts uit het veld' die ik in het kader van mijn onderzoek mocht interviewen of die hebben deelgenomen aan mijn onderzoek. ledereen was altijd even vriendelijk en behulpzaam, waardoor de datacollectie soepel verliep. Zonder jullie medewerking was dit proefschrift niet tot stand gekomen.

Als promovenda werd ik begeleid door een fantastisch team. Mijn dank gaat daarom uit naar prof. dr. Jan Hamers, prof. dr. Liz Capezuti, dr. Erik van Rossum en dr. Hilde Verbeek.

Beste Jan, door de vinger op de zere plekken te leggen en de juiste vragen te stellen, heb je mij heel wat stof tot nadenken gegeven. Ik heb heel veel aan je positief-kritische opmerkingen en suggesties gehad. Naast serieuze discussies ontbrak het plezier nooit en was er altijd ruimte voor een grap. Daarom vond (en vind) ik het ook erg fijn om voor en met je te werken. Bedankt voor alle geboden mogelijkheden.

Dear Liz, right from the heart of New York, you gave a very positive boost to my project. Your detailed feedback and your 'US-perspective' made my articles much stronger. I appreciated your support and felt blessed to have you in our multinational team. Thank you very much.

Beste Erik, toen ik solliciteerde, heb je mij aan een grondig verhoor onderworpen: of het wel iets voor me was, vier jaar te focussen op een project. Geloof me, vooral in de laatste fase van het project heb ik hier nog heel vaak aan teruggedacht. Maar dankzij jouw geduld en je niet aflatende interesse kwam ik door elke woestijn. En over 'woestijn/zand' gesproken: jij was ook degene die ervoor heeft gezorgd dat ik tijdens het schrijven van artikelen niet verzandde in onnodige uitweidingen. Ik bewonder je heldere schrijfstijl en je manier van begeleiden: je kreeg het altijd voor elkaar dat ik na een overleg met jou weer vol goede moed de deur uitliep. Ik had me geen betere begeleider kunnen wensen. Dankjewel voor alles.

Beste Hilde, jouw deur stond (en staat) altijd voor me open. Ik heb erg veel geleerd van je altijd kritische houding („Ik speel nu even advocaat van de duivel, he?”). Pas als jij tevreden was, wist ik dat het goed was. Ook je pragmatische aanpak van problemen en je relativeringsvermogen hebben me erg geholpen. Je kon bruisen van ideeën en 
enthousiasme, maar ook de nodige rust uitstralen als dat nodig was. Op momenten dat ik (weer eens) ongeduldig werd, ging jij op de rem staan. Dit heeft ervoor gezorgd dat ik nooit uit de bocht ben gevlogen. Hartelijk dank voor alles en ik kijk uit naar onze verdere samenwerking.

De leden van de beoordelingscommissie, prof. dr. Jos Schols, prof. dr. Diana Dolmans, dr. Evelyn Finnema en prof. dr. Gaby Odekerken-Schröder wil ik danken voor de bereidheid mijn proefschrift te beoordelen en zitting te nemen in de corona. I would like to thank Prof. dr. Anne Marie Rafferty for her willingness to be part of the assessment committee and the corona.

De leden van de klankbordgroep van het project Nurses on the Move dank ik voor het kritisch meedenken: Corry Ketelaars (IGZ), Sonja Kersten (V\&VN), Brigitte Verhage (VWS), Susanne Bruijns (Actiz), Marthijn Laterveer (LOC), Roger Ruijters (MeanderGroep), Trudie Severens (Sevagram), Jan Maarten Nuijens (Envida), Kina Koster (Cicero Zorggroep) en Herm Leenders (Zuyderland). Tijdens bijeenkomsten van de klankbordgroep was ook de input van Dineke Abels (ZonMw) zeer waardevol, waarvoor hartelijk dank.

Een bijzonder woord van dank gaat uit naar het team van de LPZ. Dr. Ruud Halfens, beste Ruud, ik wil jou danken voor de geboden mogelijkheid gebruik te maken van de LPZ infrastructuur. Als coauteur van drie van de zes artikelen in dit proefschrift heb je mij altijd snel van nuttige feedback voorzien. Je was niet zuinig met complimenten en leefde mee met elk afgewezen artikel. Dankjewel.

Ook Saskia Wolters en Suzanne Rijcken wil ik hierbij noemen. Beste Saskia, beste Suzanne, bedankt voor alle hulp en het wegwijs maken in de wereld van de LPZ.

Elles Lenaerts wil ik danken voor de hulp tijdens de dataverzameling. Beste Elles, dankzij jouw inspanningen verliep de dataverzameling als een trein. Alle verzamelde data moesten natuurlijk ook geanalyseerd worden. Frans Tan wil ik danken voor de ondersteuning bij de statistische analyses. Beste Frans, dankjewel voor je geduld bij het beantwoorden van mijn vele vragen.

Mijn dank gaat ook uit naar mijn vele collega's van HSR: bedankt voor de collegialiteit en de goede sfeer op de afdeling! ledereen die betrokken was bij het project Nurses on the Move wil ik danken voor het meedenken en de talrijke adviezen. Brigitte, Joanna, Suus en Arnold dank ik voor de administratieve/technische support. De junioren (en exjunioren) dank ik vooral voor de gezelligheid en de discussies tijdens de (soms te lange) pauzes in de koffiecorner. Het was heel fijn om (promotie)leed te kunnen delen en te lachen over de meest bizarre (niet werkgerelateerde) dingen, was altijd een welkome afleiding. 
Beste Nienke en Mirre, wij deelden niet alleen een kamer, maar volgens 'onze mannen' ook nog veel te veel andere dingen. Naast het harde werk en de vele inhoudelijke discussies over onze projecten was er dan ook altijd ruimte voor gezelligheid. Onze gespreksonderwerpen waren van uiteenlopende kwaliteit en de verhalen (uit het leven van andere mensen) leken vaak gestolen uit een slechte soap. Ik ben enorm trots dat ik jullie Nederlandse woordenschat heb kunnen verrijken. En ik heb overigens meegeteld: de tranen die rolden, kwamen echt wel vaker door het lachen. Lieve Nienke, vanaf het begin hebben we alle (projectgerelateerde) ups-and-downs kunnen delen. Ik ga je daarom ontzettend missen op Dub30! Ik wens je heel veel succes en geluk voor je toekomst in het Noorden van het land en ik kijk uit naar je promotie. De WhatsApp groep blijft natuurlijk bestaan. Lieve Mirre, als 'kleine Nurse' kwam je ons een jaar later vergezelschappen en al snel werd je volwardig lid van de club. Heel fijn dat je je theeroute hebt aangepast om op die manier de ineens enorme fysieke afstand tussen ons te verkleinen. Jou wens ik heel veel succes met het afronden van je proefschrift.

Beste Bram, sinds enkele weken ben jij mijn nieuwe 'roomie' en het is nu al gezellig in onze mooie, nieuwe kamer (Zo'n uitzicht zijn we natuurlijk niet gewend!). Ook jou wens ik heel veel succes met het afronden van je proefschrift. Laat je niet afleiden door alle mensen die tijdens het koffiehalen naar ons zwaaien.

Dr. Antoinette de Bont, beste Antoinette, ook zonder jou lag er nu geen boekje. Jij stond aan de wieg van mijn carrière als onderzoeker. Dankzij jou heb ik geleerd dat onderzoek doen heel leuk kan zijn. Ik ben je dan ook zeer erkentelijk voor alle geboden mogelijkheden en je vertrouwen in mij. Interviews houden bij huisartsenposten, de Inspectie of het Ministerie, een congresbezoek in Göteborg en het publiceren van een artikel zijn daar slechts enkele voorbeelden van. Ik heb een fantastische tijd gehad.

Meinen Freunden will ich dafür danken, dass sie für die nötige Ablenkung gesorgt haben! Unsere Treffen, Restaurant-, Kino- und Cafébesuche, Spieleabende, (Kurz-) Urlaube und alle andere gemeinsam verbrachte Zeit waren ein willkommener Ausgleich zu meiner Doktorarbeit. Die Tatsache, dass wir (je nach persönlichem Geschmack) damit beschäftigt sind an unterschiedlichen Orten Ehen zu schließen, Häuser zu bauen, Kinder zu bekommen und/oder Karriere zu machen, sollte uns auch zukünftig nicht davon abhalten, unsere Freundschaft zu pflegen.

Liebe Katrin, liebe Ruth, euch danke ich dafür, dass ihr mir während meiner Verteidigung als Paranimfen zur Seite stehen wollt. Ich wünsche euch viel Erfolg beim Beenden eurer Abschlussarbeiten. Die Zeit ,danach' wird dank eurer neuen ,Projekte' bestimmt nicht weniger turbulent und aufregend. Katrin, dir wünsche ich alles Gute für den Hausbau. Ruth, dir wünsche ich viel Spaß in den USA. 
Meiner Familie danke ich für das vielfältig bekundete Interesse an meiner Arbeit. Ich bin jedoch auch unglaublich froh, dass wir demnächst Familienfeste wie zum Beispiel Weihnachten feiern können ohne uns darüber zu unterhalten wann meine Doktorarbeit fertig ist, was ich ,genau' mache und was ich ,danach' machen möchte (steht übrigens alles in diesem Buch).

Liebe Mama, lieber Papa, euch bin ich unendlich dankbar für alles, was ihr mir in meinem Leben ermöglicht habt. Auch wenn ich meinen Dank nicht immer ausgesprochen habe, bin ich sehr dankbar dafür, dass ihr mich bedingungslos liebt, immer an mich glaubt und mich immer in vielfältiger Weise unterstützt. In jeder Lebenslage seid und ward ihr für mich da, habt euch in guten Zeiten mit mir gefreut und mir in schlechteren Zeiten Mut zugesprochen. Riesen Kompliment dafür, dass ihr bis zum heutigen Tag meine Launen ertragen habt! Dank euch sind wir Kinder heute starke Persönlichkeiten, die mit beiden Beinen fest im Leben stehen. Ihr sollt wissen, dass wir auch immer für euch da sein werden!

Lieber Mario, auch wenn wir unterschiedlicher nicht sein könnten, bin ich unendlich froh, dass es dich gibt! Obwohl wir erschreckend ehrlich miteinander umgehen und uns oftmals (lautstark) die Meinung sagen, wissen wir, dass wir uns immer aufeinander verlassen können. In den letzten Jahren haben deine nüchterne Denkweise, deine Kommentare und deine Anekdoten zum Thema ,Doktoranden' mich immer wieder geerdet. Dein Humor und dein Relativierungsvermögen haben mir gezeigt, dass ich das Leben nicht so ernst zu nehmen brauche.

Beste Wilfried, mijn 'lievelings Nederlander'. Jou in het Nederlands danken vind ik niet zo'n goed idee. Wie Du weißt, habe ich dir jahrelang damit gedroht, dir für dein Desinteresse an meiner Arbeit zu danken. Du hast keinen meiner Artikel gelesen und konntest auch niemandem erklären, was ich inhaltlich gemacht habe. Das Einzige, was für dich zählte war nämlich, ob ich glücklich war mit dem was ich tat. War dies nicht der Fall, hast Du meine Tränen getrocknet oder dir meine (meist banalen, sich selbst lösenden) Probleme angehört. Verglichen mit den Problemen, die wir in den letzten Jahren zusammen meistern mussten, war meine Promotion nämlich ein Klacks! Ich danke dir für den nötigen Rückhalt und die allzeit willkommene Ablenkung. Wir sollten darauf achten, dass wir auch in Zukunft genügend Gründe zum Anstoßen und zum Verreisen haben (Oder wir verreisen und stoßen dann an? Schoolreisje naar ZuidAfrika?). Bereits mein halbes Leben lang bist Du immer für mich da und zusammen sind wir ein unschlagbares Team. Ich möchte dich nicht mehr missen und kann es kaum abwarten, unseren gemeinsamen Traum vom Haus mit dir zusammen zu verwirklichen. Ich liebe dich! 

About the author 
Ramona Backhaus was born in Heinsberg, Germany, on December 22, 1988. After graduating from secondary school at 'Kreisgymnasium Heinsberg' (1999-2008), Ramona followed the bachelor program 'Health Sciences' at Maastricht University's Faculty of Health, Medicine and Life Sciences. During this bachelor, she combined her major in Health Policy and Management with a free minor (Work and Health, Care Sciences). At the Faculty of Law, she attended an elective minor in Health Law. Next to her bachelor, she followed the two-year Honours Program 'Governance of Healthcare Innovation'. In 2011, Ramona continued her education at Erasmus University Rotterdam, where she followed the master program 'Health Economics, Policy and Law' at the institute of Health Policy and Management (iBMG) and obtained her master's degree in 2012.

In November 2012, Ramona started working as a PhD candidate within the 'Living Lab in Ageing and Long-Term Care' at the Department of Health Services Research at Maastricht University. As a PhD candidate, she conducted research into the impact of nursing staff on quality of care in nursing homes. Since February 2017, she works as a research fellow within the same Living Lab, where she participates in projects that are aimed at improving quality of care, particularly from a resident/family perspective. The Living Lab is part of the CAPHRI research line 'Ageing and Long-Term Care' at Maastricht University. 
List of publications 


\section{ARTICLES}

Backhaus R, Verbeek H, Van Rossum E, Capezuti E, Hamers JPH. Blazing a Trail for Baccalaureate-Educated Registered Nurses in Nursing Homes: Experiences of Administrators and Nursing Staff. Submitted for publication.

Backhaus R, Van Rossum E, Verbeek H, Halfens RJG, Tan FES, Capezuti E, Hamers JPH. Work Environment Characteristics Associated with Quality of Care in Dutch Nursing Homes: A Cross-Sectional Study. International Journal of Nursing Studies 2017;66:1522.

Backhaus R, Van Rossum E, Verbeek H, Halfens RJG, Tan FES, Capezuti E, Hamers JPH. Relationship between the presence of baccalaureate-educated RNs and quality of care: a cross-sectional study in Dutch long-term care facilities. BMC Health Services Research 2017;17:53.

Hamers JPH, Backhaus R, Beerens HC, Van Rossum E, Verbeek H. Meer personeel leidt niet simpelweg tot betere verpleeghuiszorg; implicaties voor de praktijk en opleidingen. Onderwijs en Gezondheidszorg 2016;40:30-32.

Backhaus R, Van Rossum E, Verbeek H, Halfens RJG, Tan FES, Capezuti E, Hamers JPH. Quantity of Staff and Quality of Care in Dutch Nursing Homes : A Cross-Sectional Study. The Journal of Nursing Home Research Sciences 2016;2:90-93.

Backhaus R, Verbeek H, Van Rossum E, Capezuti E, Hamers JPH. Future Distinguishing Competencies of Baccalaureate-Educated Registered Nurses in Nursing Homes. Geriatric Nursing 2015;36:438-444.

Backhaus R, Verbeek H, Van Rossum E, Capezuti E, Hamers JPH. Nurse Staffing Impact on Quality of Care in Nursing Homes: A Systematic Review of Longitudinal Studies. Journal of the American Medical Directors Association 2014;15:383-393.

\section{REPORTS}

Hamers JPH, Backhaus R, Beerens HC, Van Rossum E, Verbeek H. Meer is niet per se beter. De relatie tussen personele inzet en kwaliteit van zorg in verpleeghuizen. Maastricht: Datawyse/Universitaire Pers Maastricht, 2016. 


\section{CONFERENCE ABSTRACTS}

Backhaus R, Verbeek H, Van Rossum E, Capezuti E, Hamers JPH. Baccalaureate-Educated Registered Nurses in Nursing Homes: Positioning and Role Implementation. 21st IAGG World Congress of Gerontology and Geriatrics, 23-27 July 2017, San Francisco, CA, USA. Accepted for presentation.

Backhaus R, Verbeek H, Van Rossum E, Capezuti E, Hamers JPH. Baccalaureate-Educated Registered Nurse Competencies in Future Nursing Homes. Caring for Older People: How Can We Do The Right Things Right? 4-7 October 2016, Rotterdam, The Netherlands. Journal of Advanced Nursing 2016(suppl. 1):48.

Backhaus R, Van Rossum E, Verbeek H, Halfens RJG, Tan FES, Capezuti E, Hamers JPH. Staff-Related Work Environment Characteristics and Quality of Care in Dutch Nursing Homes. Caring for Older People: How Can We Do The Right Things Right? 4-7 October 2016, Rotterdam, The Netherlands. Journal of Advanced Nursing 2016(suppl. 1):87-88.

Backhaus R, Verbeek H, Van Rossum E, Capezuti E, Hamers JPH. Future Distinguishing Competencies of Bachelor-Educated Registered Nurses in Nursing Homes. International Nursing Home Research Conference, 2-3 December 2015, Toulouse, France. The Journal of Nursing Home Research Sciences 2015;1:11.

Backhaus R, Verbeek H, Van Rossum E, Capezuti E, Hamers JPH. Future Distinguishing Competencies of Baccalaureate-Educated Registered Nurses in Nursing Homes. 68th Annual scientific meeting of the Gerontological Society of America, 18-22 November 2015, Orlando, FL, USA. Gerontologist 2015;55(suppl. 2):142-3.

Backhaus R, Van Rossum E, Verbeek H, Capezuti E, Hamers JPH. Relationship Between Bachelor-RN Staffing and Quality of Care in Dutch Long-Term Care Facilities. 68th Annual scientific meeting of the Gerontological Society of America, 18-22 November 2015, Orlando, FL, USA. Gerontologist 2015;55(suppl. 2):806.

Backhaus R, Verbeek H, Van Rossum E, Capezuti E, Hamers JPH. Future Distinguishing Competencies of Baccalaureate-Educated Registered Nurses in Nursing Homes. EANS summer conference 'Improving access to healthcare', 8-9 July 2015, Barcelona, Spain. BMC Nursing 2015;14(suppl. 1):S10.

Backhaus R, Verbeek H, Van Rossum E, Capezuti E, Hamers JPH. Toekomstige onderscheidende competenties van $\mathrm{HBO}$-verpleegkundigen in verpleeghuizen. 13e Nationaal Gerontologiecongres, 2 oktober 2015, Ede, the Netherlands. Tijdschrift voor Gerontologie en Geriatrie 2015;46:226. 
Backhaus R, Verbeek H, Van Rossum E, Capezuti E, Hamers JPH. Nurse Staffing Impact on Quality of Care in Nursing Homes: Systematic Review on Longitudinal Studies. 67th Annual scientific meeting of the Gerontological Society of America, 5-9 November 2014, Washington DC, USA. Program Book GSA Meeting 2014.

Verbeek H, Backhaus R, Van Rossum E, Hamers JPH. Skills and Competencies of Nursing Staff in Long Term Care Providing Person Centered Care. 67th Annual scientific meeting of the Gerontological Society of America, 5-9 November 2014, Washington DC, USA. Program Book GSA Meeting 2014.

Backhaus R, Verbeek H, Van Rossum E, Capezuti E, Hamers JPH. Effect of Nurse Staffing on Quality of Care in Nursing Homes: A Systematic Review. 14th European Doctoral Conference in Nursing Science 'Nursing science: one goal, different features', 12-13 September 2014. Verpleegkunde 2014;29:21.

Backhaus R, Verbeek H, Van Rossum E, Capezuti E, Hamers JPH. Effect of Staffing on Quality of Care in US Nursing Homes. Late Breaker Poster Session Program Book GSA Meeting 2013. 
Living lab in ageing and long-term care 


\section{LIVING LAB IN AGEING AND LONG-TERM CARE}

This thesis is part of the Living Lab in Ageing and Long-Term Care, a formal and structural multidisciplinary network consisting of Maastricht University, 7 long-term care organizations (Cicero Zorggroep, Envida, Mosae Zorggroep, MeanderGroep ZuidLimburg, Sevagram, Vivantes and Zuyderland) and Zuyd University of Applied Sciences, all located in the southern part of the Netherlands. In the Living Lab we aim to improve quality of care and quality of life for older people via a structural multidisciplinary collaboration between research, policy, education and practice. Practitioners (such as nurses, physicians, psychologists, physio- and occupational therapists), work together with managers, researchers, students, teachers and older people themselves to develop and test innovations in long-term care.

\section{ACADEMISCHE WERKPLAATS OUDERENZORG ZUID-LIMBURG}

Dit proefschrift is onderdeel van de Academische Werkplaats Ouderenzorg ZuidLimburg, een structureel, multidisciplinair samenwerkingsverband tussen de Universiteit Maastricht, 7 zorgorganisaties (Cicero Zorggroep, Envida, Mosae Zorggroep, MeanderGroep Zuid-Limburg, Sevagram, Vivantes en Zuyderland) en Zuyd Hogeschool. In de werkplaats draait het om het verbeteren van de kwaliteit van leven en zorg voor ouderen. Zorgverleners (zoals verpleegkundigen, verzorgenden, artsen, psychologen, fysio- en ergotherapeuten), beleidsmakers, onderzoekers, studenten en ouderen zelf wisselen kennis en ervaring uit. Daarnaast toetsen en evalueren we vernieuwingen in de dagelijkse zorg. Praktijk, beleid, onderzoek en onderwijs gaan hierbij hand in hand. 


\section{PHD-THESES LIVING LAB IN AGEING AND LONG-TERM CARE / PROEFSCHRIFTEN ACADEMISCHE WERKPLAATS OUDERENZORG ZUID- LIMBURG}

Ramona Backhaus. Thinking beyond numbers. Nursing staff and quality of care in nursing homes. 2017

Irma Everink. Geriatric rehabilitation. Development, implementation and evaluation of an integrated care pathway for older patients with complex health problems. 2017

Martin Van Leen. Prevention of pressure ulcers in nursing homes, a big challenge. 2017

Mariëlle Daamen-Van der Velden. Heart failure in nursing home residents. Prevalence, diagniosis and treatment. 2016

Armand Rondas. Prevalence and assessment of (infected) chronic wounds. 2016

Hanneke Beerens. Adding life to years. Quality of life of people with dementia receiving long-term care. 2016 (Cum Laude)

Donja Mijnarends. Sarcopenia: a rising geriatric giant. Health and economic outcomes of community-dwelling older adults with sarcopenia. 2016

Tanja Dorresteijn. A home-based program to manage concerns about falls. Feasibility, effects and costs of a cognitive behavioral approach in community-dwelling, frail older people. 2016

Basema Afram. From home towards the nursing home in dementia. Informal caregivers' perspectives on why admission happens and what they need. 2015

Noemi Van Nie-Visser. Malnutrition in nursing home residents in the Netherlands, Germany and Austria. Exploring and comparing influencing factors. 2014

Esther Meesterberends. Pressure ulcer care in the Netherlands versus Germany 01. What makes the difference? 2013

Math Gulpers. EXBELT: expelling belt restraints from psychogeriatric nursing homes. 2013

Hilde Verbeek. Redesigning dementia care. An evaluation of small-scale homelike care environments. 2011 
Judith Meijers. Awareness of malnutrition in health care, the Dutch perspective. 2009 Ans Bouman. A home visiting program for older people with poor health. 2009.

Monique Du Moulin. Urinary incontinence in primary care, diagnosis and interventions. 2008

Anna Huizing. Towards restraint free care for psychogeriatric nursing home residents. 2008

Pascalle Van Bilsen. Care for the elderly, an exploration of perceived needs, demands and service use. 2008

Rixt Zijlstra. Managing concerns about falls. Fear of falling and avoidance of activity in older people. 2007

Sandra Zwakhalen. Pain assessment in nursing home residents with dementia. 2007 
It is a persistent belief that more nursing staff is needed to improve quality of care in nursing homes. An increase in the number of staff is presumed to affect the quality of care and quality of life of nursing home residents. But is it the quantity of staff that matters? Or do we have to think beyond numbers, focusing on what nursing staff is doing in every day care practice to improve quality of care in nursing homes?

In this dissertation, Ramona Backhaus reports the results of her research on nursing staff and quality of care in nursing homes. She provides insight into the relationship between direct nursing care staffing, staff-related work environment characteristics (e.g., team work) and quality of care in nursing homes. In addition, she pays particular attention to the competencies, tasks and employment of baccalaureate-educated registered nurses. They are expected to serve as informal leaders that have the ability to lead improvements and redesign practice environments in nursing homes. The results of this dissertation have several implications for practice, education, policy and research. 